\title{
A First Look at BISTRO Observations of the Oph-A core
}

DOI:

10.3847/1538-4357/aabd82

\section{Document Version}

Final published version

Link to publication record in Manchester Research Explorer

\section{Citation for published version (APA):}

Kwon, J., Doi, Y., Tamura, M., Matsumura, M., Pattle, K., Berry, D., Sadavoy, S., Matthews, B. C., WardThompson, D., Hasegawa, T., Furuya, R. S., Pon, A., Francesco, J. D., Arzoumanian, D., Hayashi, S. S., Kawabata, K. S., Onaka, T., Choi, M., Kang, M., ... Zenko, T. (2018). A First Look at BISTRO Observations of the Oph-A core. Astrophysical Journal, 859(1), [4]. https://doi.org/10.3847/1538-4357/aabd82

\section{Published in:}

Astrophysical Journal

\section{Citing this paper}

Please note that where the full-text provided on Manchester Research Explorer is the Author Accepted Manuscript or Proof version this may differ from the final Published version. If citing, it is advised that you check and use the publisher's definitive version.

\section{General rights}

Copyright and moral rights for the publications made accessible in the Research Explorer are retained by the authors and/or other copyright owners and it is a condition of accessing publications that users recognise and abide by the legal requirements associated with these rights.

\section{Takedown policy}

If you believe that this document breaches copyright please refer to the University of Manchester's Takedown Procedures [http://man.ac.uk/04Y6Bo] or contact uml.scholarlycommunications@manchester.ac.uk providing relevant details, so we can investigate your claim.

\section{OPEN ACCESS}




\section{A First Look at BISTRO Observations of the $\rho$ Oph-A core}

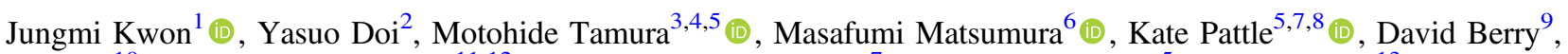
Sarah Sadavoy ${ }^{10}$ (1) , Brenda C. Matthews ${ }^{11,12}$ (1) , Derek Ward-Thompson $^{7}$ (1) , Tetsuo Hasegawa ${ }^{5}$, Ray S. Furuya ${ }^{13}$ (1), Andy Pon ${ }^{14}$ (D), James Di Francesco ${ }^{11,12}$, Doris Arzoumanian ${ }^{15}$, Saeko S. Hayashi ${ }^{16}$, Koji S. Kawabata ${ }^{17,18,19}$ (10), Takashi Onaka ${ }^{3}$ (10), Minho Choi ${ }^{20}$,

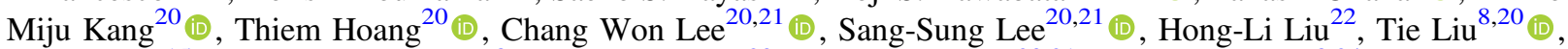

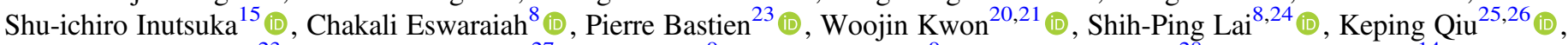
Simon Coudé ${ }^{23}$ (i) , Erica Franzmann ${ }^{27}$, Per Friberg ${ }^{9}$, Sarah F. Graves ${ }^{9}$ (i), Jane S. Greaves ${ }^{28}$ (iD), Martin Houde ${ }^{14}$ (1),

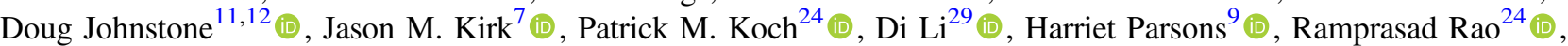

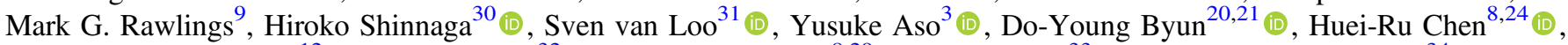
Mike C.-Y. Chen ${ }^{12}$, Wen Ping Chen ${ }^{32}$ (1), Tao-Chung Ching ${ }^{8,29}$, Jungyeon $\mathrm{Cho}^{33}$ (1), Antonio Chrysostomou ${ }^{34}$ (1), Eun Jung Chung ${ }^{20}$ (1) , Emily Drabek-Maunder ${ }^{28}$, Stewart P. S. Eyres ${ }^{7}$ (D), Jason Fiege ${ }^{27}$, Rachel K. Friesen ${ }^{35}$ (i), Gary Fuller ${ }^{36}$ (1), Tim Gledhill $^{34}$ (D) , Matt J. Griffin ${ }^{28}$, Qilao Gu ${ }^{22}$, Jennifer Hatchell ${ }^{37}$ (D), Wayne Holland ${ }^{38,39}$, Tsuyoshi Inoue ${ }^{15}$, Kazunari Iwasaki ${ }^{40}$, Il-Gyo Jeong ${ }^{20}$, Ji-hyun Kang ${ }^{20}(1)$, Sung-ju Kang ${ }^{20}\left(10\right.$, Francisca Kemper ${ }^{24}(1)$, Gwanjeong Kim $^{20,21}$, Jongsoo Kim ${ }^{20,21}$, Kee-Tae Kim ${ }^{20}$ (1) , Kyoung Hee Kim ${ }^{41}$ (1), Mi-Ryang $\operatorname{Kim}^{20}$ (1), Shinyoung Kim ${ }^{20,21}$, Kevin M. Lacaille ${ }^{42,43}$ (1), Jeong-Eun Lee ${ }^{44}$ (1), Dalei Li ${ }^{45}$, Hua-bai Li $i^{22}$, Junhao Liu ${ }^{25,26}$, Sheng-Yuan Liu ${ }^{24}$ (1), A-Ran Lyo ${ }^{20}$ (1), Steve Mairs ${ }^{9}$ (1), Gerald H. Moriarty-Schieven ${ }^{11}$, Fumitaka Nakamura $^{46,47}$ (i) , Hiroyuki Nakanishi ${ }^{1,30}$, Nagayoshi Ohashi ${ }^{16}$ (1) , Nicolas Peretto $^{28}$, Tae-Soo Pyo ${ }^{16,47}$ (i), Lei Qian ${ }^{29}$ (i), Brendan Retter $^{28}$, John Richer ${ }^{48,49}$ (10), Andrew Rigby ${ }^{28}$, Jean-Franois Robitaille ${ }^{36}$, Giorgio Savini ${ }^{50}$, Anna M. M. Scaife ${ }^{36}$ (1), Archana Soam $^{20}$ (10), Ya-Wen Tang ${ }^{24}$ (10), Kohji Tomisaka ${ }^{46,47}$ (1) , Hongchi Wang ${ }^{51}$ (D), Jia-Wei Wang ${ }^{8}$ (1) , Anthony P. Whitworth ${ }^{28}$, Hsi-Wei Yen ${ }^{24,52}$ (1), Hyunju Yoo ${ }^{33}$, Jinghua Yuan ${ }^{29}$ (1), Chuan-Peng Zhang ${ }^{29}$ (1), Guoyin Zhang ${ }^{29}$, Jianjun Zhou ${ }^{45}$, Lei Zhu ${ }^{29}$, Philippe André ${ }^{53}$, C. Darren Dowell ${ }^{54}$, Sam Falle ${ }^{55}$, Yusuke Tsukamoto ${ }^{56}$, Takao Nakagawa ${ }^{1}$ (1), Yoshihiro Kanamori ${ }^{2}$, Akimasa Kataoka $^{46}\left(\mathbb{D}\right.$, Masato I. N. Kobayashi ${ }^{15}$ (1D), Tetsuya Nagata ${ }^{57}$, Hiro Saito ${ }^{58}$, Masumichi Seta ${ }^{59}$, and Tetsuya Zenko ${ }^{57}$

${ }^{1}$ Institute of Space and Astronautical Science, Japan Aerospace Exploration Agency, 3-1-1 Yoshinodai, Chuo-ku, Sagamihara, Kanagawa 252-5210, Japan jungmi.kwon@ir.isas.jaxa.jp

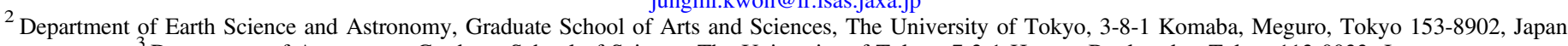

${ }^{3}$ Department of Astronomy, Graduate School of Science, The University of Tokyo, 7-3-1 Hongo, Bunkyo-ku, Tokyo 113-0033, Japan

Astrobiology Center, National Institutes of Natural Sciences, 2-21-1 Osawa, Mitaka, Tokyo 181-8588, Japan

${ }^{5}$ National Astronomical Observatory of Japan, National Institutes of Natural Sciences, Osawa, Mitaka, Tokyo 181-8588, Japan

${ }^{6}$ Faculty of Education, Kagawa University, Saiwai-cho 1-1, Takamatsu, Kagawa, 760-8522, Japan 7 Jeremiah Horrocks Institute, University of Central Lancashire, Preston PR1 2HE, UK

${ }^{8}$ Institute of Astronomy and Department of Physics, National Tsing Hua University, Hsinchu 30013, Taiwan

${ }^{9}$ East Asian Observatory, 660 N. A 'ohōkū Place, University Park, Hilo, HI 96720, USA

${ }^{10}$ Harvard-Smithsonian Center for Astrophysics, 60 Garden Street, Cambridge, MA 02138, USA

${ }^{11}$ NRC Herzberg Astronomy and Astrophysics, 5071 West Saanich Road, Victoria, BC V9E 2E7, Canada

12 Department of Physics and Astronomy, University of Victoria, Victoria, BC V8P 1A1, Canada

${ }^{13}$ Institute of Liberal Arts and Sciences Tokushima University, Minami Jousanajima-machi 1-1, Tokushima 770-8502, Japan

${ }^{14}$ Department of Physics and Astronomy, The University of Western Ontario, 1151 Richmond Street, London N6A 3K7, Canada

${ }^{15}$ Department of Physics, Graduate School of Science, Nagoya University, Furo-cho, Chikusa-ku, Nagoya 464-8602, Japan

${ }^{16}$ Subaru Telescope, National Astronomical Observatory of Japan, 650 N. A 'ohōkū Place, Hilo, HI 96720, USA

${ }^{17}$ Hiroshima Astrophysical Science Center, Hiroshima University, Kagamiyama 1-3-1, Higashi-Hiroshima, Hiroshima 739-8526, Japan

${ }^{18}$ Department of Physics, Hiroshima University, Kagamiyama 1-3-1, Higashi-Hiroshima, Hiroshima 739-8526, Japan

${ }^{19}$ Core Research for Energetic Universe (CORE-U), Hiroshima University, Kagamiyama 1-3-1, Higashi-Hiroshima, Hiroshima 739-8526, Japan

${ }^{20}$ Korea Astronomy and Space Science Institute, 776 Daedeokdae-ro, Yuseong-gu, Daejeon 34055, Republic of Korea

${ }^{21}$ Korea University of Science and Technology, 217 Gajang-ro, Yuseong-gu, Daejeon 34113, Republic of Korea

${ }^{22}$ Department of Physics, The Chinese University of Hong Kong, Shatin, N.T., Hong Kong

${ }^{23}$ Centre de recherche en astrophysique du Québec \& département de physique, Université de Montréal, C.P. 6128, Succ. Centre-ville, Montréal, QC H3C 3J7, Canada

${ }^{24}$ Academia Sinica Institute of Astronomy and Astrophysics, P.O. Box 23-141, Taipei 10617, Taiwan

${ }^{25}$ School of Astronomy and Space Science, Nanjing University, 163 Xianlin Avenue, Nanjing 210023, People's Republic of China

${ }^{26}$ Key Laboratory of Modern Astronomy and Astrophysics (Nanjing University), Ministry of Education, Nanjing 210023, People's Republic of China

${ }^{27}$ Department of Physics and Astronomy, The University of Manitoba, Winnipeg, MB R3T 2N2, Canada

${ }^{2}$ School of Physics and Astronomy, Cardiff University, The Parade, Cardiff, CF24 3AA, UK

${ }^{29}$ National Astronomical Observatories, Chinese Academy of Sciences, A20 Datun Road, Chaoyang District, Beijing 100012, People's Republic of China ${ }^{30}$ Kagoshima University, 1-21-35 Korimoto, Kagoshima, Kagoshima 890-0065, Japan

31 School of Physics and Astronomy, University of Leeds, Woodhouse Lane, Leeds LS2 9JT, UK 32 Institute of Astronomy, National Central University, Chung-Li 32054, Taiwan

33 Department of Astronomy and Space Science, Chungnam National University, 99 Daehak-ro, Yuseong-gu, Daejeon 34134 , Republic of Korea

34 School of Physics, Astronomy \& Mathematics, University of Hertfordshire, College Lane, Hatfield, Hertfordshire AL10 9AB, UK

National Radio Astronomy Observatory, 520 Edgemont Rd., Charlottesville, VA 22903, USA

36 Jodrell Bank Centre for Astrophysics, School of Physics and Astronomy, University of Manchester, Oxford Road, Manchester, M13 9PL, UK ${ }^{37}$ Physics and Astronomy, University of Exeter, Stocker Road, Exeter EX4 4QL, UK

${ }^{38}$ UK Astronomy Technology Centre, Royal Observatory, Blackford Hill, Edinburgh EH9 3HJ, UK

${ }^{39}$ Institute for Astronomy, University of Edinburgh, Royal Observatory, Blackford Hill, Edinburgh EH9 3HJ, UK

${ }^{40}$ Department of Earth and Space Science, Osaka University, Machikaneyama-cho, Toyonaka, Osaka 560-0043, Japan

41 Department of Earth Science Education, Kongju National University, 56 Gongjudaehak-ro, Gongju-si 32588, Republic of Korea

${ }^{42}$ Department of Physics and Astronomy, McMaster University, Hamilton, ON L8S 4M1, Canada

${ }^{43}$ Department of Physics and Atmospheric Science, Dalhousie University, Halifax, NS B3H 4R2, Canada 


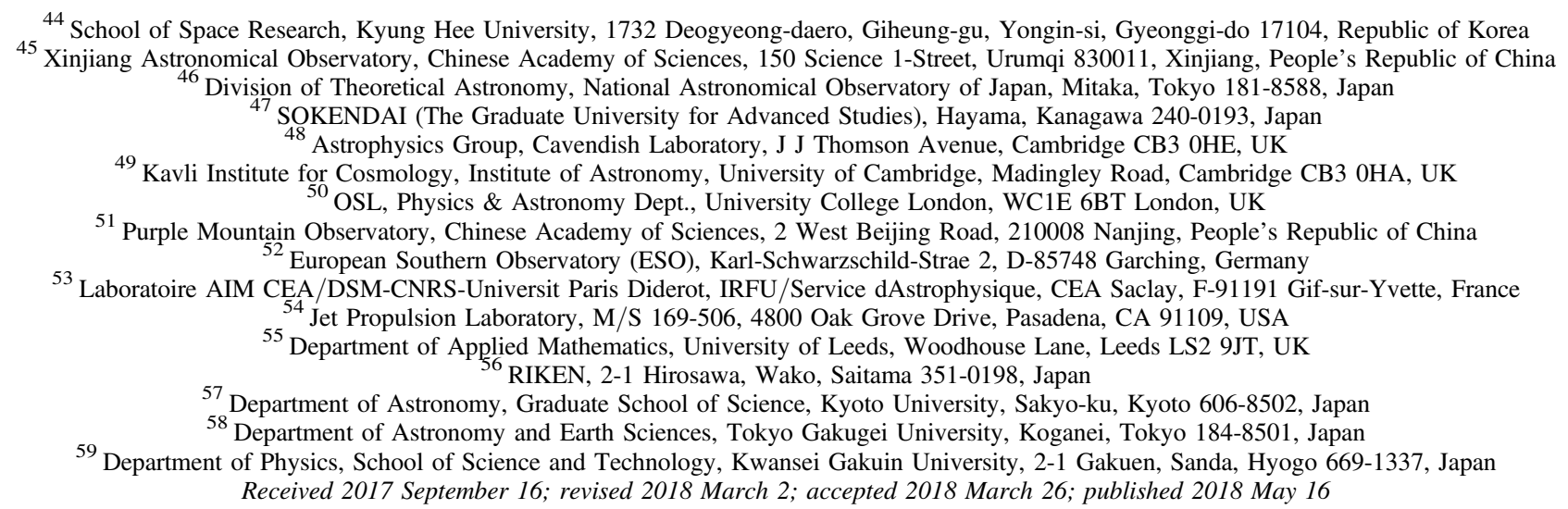

\begin{abstract}
We present $850 \mu \mathrm{m}$ imaging polarimetry data of the $\rho$ Oph-A core taken with the Submillimeter Common-User Bolometer Array-2 (SCUBA-2) and its polarimeter (POL-2) as part of our ongoing survey project, $\boldsymbol{B}$-fields In STar forming RegiOns (BISTRO). The polarization vectors are used to identify the orientation of the magnetic field projected on the plane of the sky at a resolution of $0.01 \mathrm{pc}$. We identify 10 subregions with distinct polarization fractions and angles in the $0.2 \mathrm{pc} \rho$ Oph-A core; some of them can be part of a coherent magnetic field structure in the $\rho$ Oph region. The results are consistent with previous observations of the brightest regions of $\rho$ Oph-A, where the degrees of polarization are at a level of a few percent, but our data reveal for the first time the magnetic field structures in the fainter regions surrounding the core where the degree of polarization is much higher $(>5 \%)$. A comparison with previous near-infrared polarimetric data shows that there are several magnetic field components that are consistent at near-infrared and submillimeter wavelengths. Using the Davis-Chandrasekhar-Fermi method, we also derive magnetic field strengths in several subcore regions, which range from approximately 0.2 to $5 \mathrm{mG}$. We also find a correlation between the magnetic field orientations projected on the sky and the core centroid velocity components.
\end{abstract}

Key words: circumstellar matter - ISM: individual objects (Ophiuchi) - ISM: structure - polarization - radio continuum: ISM - stars: formation

Supporting material: machine-readable table

\section{Introduction}

Stars form in dense and cold molecular clouds, and it has long been considered that magnetic fields may play significant roles in various stages of star formation (e.g., Shu et al. 1987; Bergin \& Tafalla 2007; McKee \& Ostriker 2007; André et al. 2013). Near-infrared linear polarimetry is one of the traditional methods of tracing magnetic field structure in order to measure the magnetic fields in denser regions than those traced by optical polarimetry, which are directly related to the star formation process (e.g., Davis \& Greenstein 1951; Lazarian 2007). The magnetic field has been successfully traced in dense regions of several molecular clouds (e.g., Wilking et al. 1979; Tamura et al. 1987, 1988, 2007; Kwon et al. 2010, 2011, 2015; Cashman \& Clemens 2014; Santos et al. 2014; Ward-Thompson et al. 2017a). Polarization at near-infrared wavelengths, however, relies on measurements of dust extinction from background stars and as such cannot well trace magnetic fields in denser substructures, like filaments and cores within clouds. As these structures are directly linked to star formation, it is vital to measure their magnetic fields. Observations of dust polarization from thermal emission at farinfrared and (sub)millimeter wavelengths can trace these high column densities and probe how the magnetic field influences the star formation process (e.g., Tamura et al. 1999; Pattle et al. 2015; Ward-Thompson et al. 2017b; see also Soler et al. 2016).

The $\rho$ Ophiuchi (hereafter $\rho$ Oph) dark cloud complex is one of the closest star-forming regions at a distance of approximately 120-165 pc (e.g., Chini 1981; de Geus et al. 1989; Knude \& Hog 1998; Rebull et al. 2004; Loinard et al. 2008; Lombardi et al. 2008; Mamajek 2008; Snow et al. 2008; Ortiz-León et al. 2017). It has also been widely studied (see Kwon et al. 2015; see also Wilking et al. 2008 for a reference summary). It is a nearby region of clustered low- to intermediate-mass star formation (e.g., Wilking et al. 2008) and is heavily influenced by the nearby Sco OB2 association (Vrba 1977; Loren 1989a, 1989b; Kwon et al. 2015). It was observed as part of the JCMT Gould Belt Legacy Survey (WardThompson et al. 2007), the Herschel Gould Belt Survey (André et al. 2010), and the Spitzer Gould Belt Survey (Evans et al. 2009). In the main body of $\rho$ Oph, detailed $\mathrm{DCO}^{+}$ observations have identified several very dense, cold cores labeled A-F (Loren \& Wootten 1986; Loren et al. 1990), and $\rho$ Oph-A appears to be the warmest among these cores (Zeng et al. 1984). The first submillimeter continuum observations of the $\rho$ Oph-A core region were obtained by Ward-Thompson et al. (1989). Many subcores in this region were identified (e.g., Motte et al. 1998; André et al. 2007) and will be described in Section 5. In this paper, we use the term "core" for the $\rho$ Oph-A complex and the term "subcore" for the smaller condensations within it.

Here we present new observations of the $\rho$ Oph-A core in dust polarization from the James Clerk Maxwell Telescope (JCMT) as part of the $\boldsymbol{B}$-fields In STar forming RegiOns (BISTRO) survey (Ward-Thompson et al. 2017a). The JCMT 
magnetic field survey of the Gould Belt clouds is a large-scale project that aims to map the submillimeter polarization of the dust thermal emission in the densest parts of all of the Gould Belt star-forming regions. The combination of the Submillimeter Common-user Bolometer Array-2 (SCUBA-2; Holland et al. 2013) and its polarimeter POL-2 (P. Bastien et al. 2018, in preparation) enables deep submillimeter polarimetry and is one of the most powerful instruments to reveal the magnetic field structure in star-forming regions, thanks to its high sensitivity and high resolution (Ward-Thompson et al. 2017a; Pattle et al. 2017).

The paper is outlined as follows. In Section 2, we describe the submillimeter observations, and the SCUBA-2/POL-2 data reduction is described in Section 3. In Section 4, we present the results of the submillimeter imaging polarimetry. In Section 5, we discuss the magnetic field structure related to the starforming activity in the $\rho$ Oph-A core region. A summary is given in Section 6.

\section{Observations}

Continuum observations of $\rho$ Oph-A at $850 \mu \mathrm{m}$ were made by inserting POL-2 into the optical path of SCUBA-2 between 2016 April 15 and 2016 April 24. The region was observed in 20 sets of 41 minute observations, and among the 20 sets, two sets with bad-quality data were excluded. Note that the BISTRO time was allocated to take place during Band 2 weather $\left(0.05<\tau_{225 \mathrm{GHz}}<0.08\right)$. The observations were made using fully sampled $12^{\prime}$ diameter circular regions with a resolution of 14 ". 1 using a version of the SCUBA-2 DAISY mapping mode (Holland et al. 2013) optimized for POL-2 observations. The POL-2 DAISY scan pattern produces a central $3^{\prime}$ diameter region of approximately even coverage, with noise increasing to the edge of the map. The mode has a scan speed of $8^{\prime \prime} \mathrm{s}^{-1}$, with a half-waveplate rotation speed of $2 \mathrm{~Hz}$ (Friberg et al. 2016). Continuum polarimetric observations were simultaneously taken at $450 \mu \mathrm{m}$ with a resolution of 9 ". 6 . In this paper, we discuss the $850 \mu \mathrm{m}$ data only.

\section{Data Reduction}

The $850 \mu \mathrm{m}$ POL-2 data were reduced in a three-stage process using the pol2map routine (the version updated on 2017 May 27) in SMURF (Berry et al. 2005; Chapin et al. 2013), which we summarize here. The POL-2 data reduction is described in detail by Bastien et al. (2018, in preparation). See also Ward-Thompson et al. (2017a) for a brief summary.

In the first stage, the raw bolometer timestreams for each observation are converted into separate Stokes $Q, U$, and $I$ timestreams using the process calcqu. An initial Stokes $I$ map is created from the $I$ timestream from each observation using the iterative map-making routine makemap. For each reduction, areas of astrophysical emission are defined using a signal-tonoise-based mask determined iteratively by makemap. Areas outside this masked region are set to zero until the final iteration of makemap (see Mairs et al. 2015 for a detailed description of the role of masking in SCUBA-2 data reduction). Each map is compared to the first map in the sequence to determine a set of relative pointing corrections. The individual $I$ maps are coadded to produce an initial I map of the region.

In the second stage, an improved Stokes $I$ map is created from the $I$ timestreams of each observation using makemap.
The initial $I$ map (described above) is used to generate a fixed signal-to-noise-based mask for all iterations of makemap. The pointing corrections determined in Stage 1 are applied during the map-making process. In all cases, the polarized sky background is estimated by doing a principal component analysis (PCA) of the $I, Q$, and $U$ timestreams to identify components that are common to multiple bolometers. In the first stage, the 50 most correlated components are removed at each iteration. In the second stage, 150 components are removed at each iteration, resulting in smaller changes in the map between iterations and lower noise in the final map. All of the individual improved $I$ maps are coadded to form the final output $I$ map.

In the third stage, the Stokes $Q$ and $U$ maps and the final vector catalog are created. Individual $Q$ and $U$ maps are reduced separately using makemap and are created from the timestreams created in Stage 1, using the same mask based on the initial Stokes $I$ map as was used in Stage 2 and the pointing offsets determined in Stage 1. Correction for instrumental polarization is performed based on the final output $I$ map. The sets of individual $Q$ and $U$ maps are then coadded to create final $Q$ and $U$ maps. The final coadded Stokes $Q, U$, and $I$ maps are used to create an output vector catalog that includes the coordinates (J2000.0), values of Stokes parameters, degrees of polarization $(P \pm \delta P)$, and polarization position angles $(\theta \pm \delta \theta)$. Therefore, it uses exactly the same map-making procedure to create all three maps-Stokes $Q, U$, and $I$-and the spatial frequencies present in the three maps are all in common.

The output $Q, U$, and $I$ maps are gridded to $4^{\prime \prime}$ pixels and calibrated in mJy beam ${ }^{-1}$ using a flux conversion factor (FCF) of $725 \mathrm{Jy} \mathrm{pW}^{-1}$ (the standard SCUBA-2 $850 \mu \mathrm{m}$ FCF of $537 \mathrm{Jy} \mathrm{pW}^{-1}$ multiplied by a factor of 1.35 to account for additional losses from POL-2; cf. Dempsey et al. 2013; Friberg et al. 2016). The output vectors are debiased using the mean of their $Q$ and $U$ variances to remove statistical biasing in regions of low signal-to-noise ( $\mathrm{S} / \mathrm{N}$; see Equation (3) below).

The raw degree of polarization, $P^{\prime}$, and the uncertainty in the degree of polarization, $\delta P$, can be calculated from the expressions

$$
P^{\prime}=\frac{\sqrt{Q^{2}+U^{2}}}{I} \times 100 \%
$$

and

$$
\delta P^{\prime}=\left(P^{\prime} I^{2}\right)^{-1} \sqrt{\left(Q^{2} \delta Q^{2}+U^{2} \delta U^{2}+\left(P^{\prime}\right)^{4} I^{2} \delta I^{2}\right)} .
$$

Note that in the pipeline software (without debiasing; see below), $P^{\prime} I$ is first calculated from $Q, \delta Q, U$, and $\delta U$, then $\delta P^{\prime}$ is calculated from $I, \delta I, Q, U$, and $\delta P I$. The expression here is identical to the formula in the pipeline but tries to show the dependence on the errors of $I, Q$, and $U$.

As mentioned, a bias exists that tends to increase the polarization percentage value, even when Stokes $Q$ and $U$ are consistent with a value of zero, because the polarization percentage is forced to be positive (Vaillancourt 2006). To mitigate this problem, approximate debiased values are calculated in the pipeline, assuming $\delta Q \sim \delta U$, as

$$
P I=\sqrt{Q^{2}+U^{2}-0.5\left(\delta Q^{2}+\delta U^{2}\right)},
$$




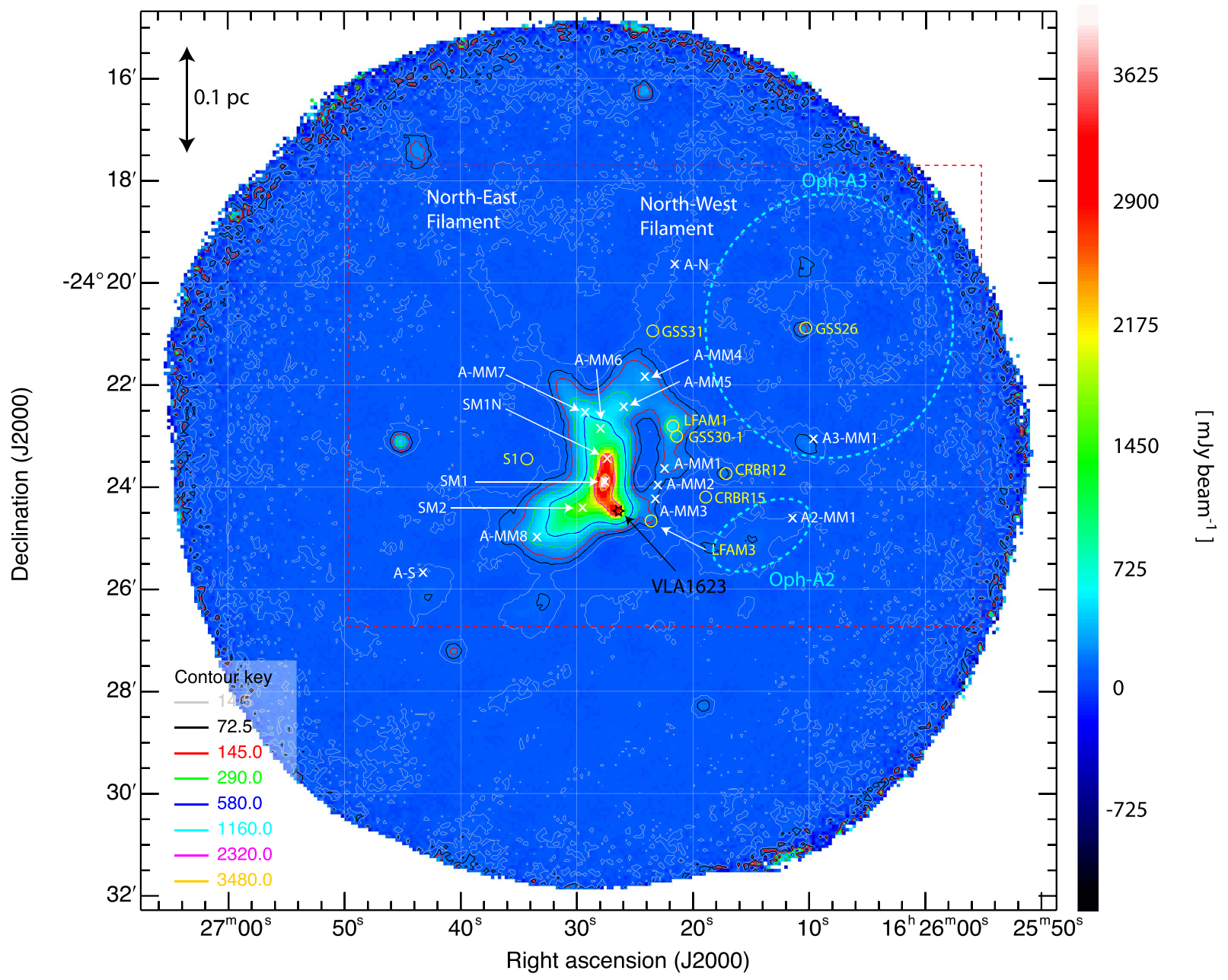

Figure 1. Stokes $I$ image (linear scale) of the $\rho$ Oph-A field centered on Oph-A1 (Motte et al. 1998) obtained using the JCMT with SCUBA-2/POL-2. Notable sources and features in this region are labeled. The spatial resolution is 14 !" 1 , or approximately $0.01 \mathrm{pc}$, assuming a distance to the Oph cloud of $140 \mathrm{pc}$. The white crosses indicate the positions of the starless condensations identified by Motte et al. (1998) in the dust continuum at $1.2 \mathrm{~mm}$ (corresponding to the red dashed rectangle region), the yellow circles indicate the positions of young embedded stars, and the black star indicates the position of VLA 1623. The cyan dashed circles indicate Oph-A2 and Oph-A3, defined by Motte et al. (1998). The contour levels are arbitrarily chosen to emphasize the Oph-A core, and their keys are shown in units of mJy beam ${ }^{-1}$.

and the degree of polarization $P$ is derived from the polarized intensity $P I$ as

$$
P=P I / I \text {. }
$$

The polarization position angles, $\theta$, and their errors, $\delta \theta$, can then be calculated by the following relations:

$$
\theta=\frac{1}{2} \tan ^{-1} \frac{U}{Q}
$$

and

$$
\begin{aligned}
\delta \theta= & 0.5 \times \sqrt{Q^{2} \times \delta U^{2}+U^{2} \times \delta Q^{2}} /\left(Q^{2}+U^{2}\right) \\
& \times 180^{\circ} / \pi .
\end{aligned}
$$

The data reduction process described above derives the Stokes I map from the same POL-2 observations that are used to derive the Stokes $Q$ and $U$ maps. A consequence of this is that the FCFs for the Stokes $I, Q$, and $U$ maps are then all equal and so cancel out when calculating the fractional polarization. As a result, the $I$ and the $Q$ and $U$ maps necessarily have exactly the same spatial scales. Earlier versions of the POL-2 pipeline software derived the Stokes I map from separate observations taken without POL-2 in the beam, resulting in the $I$ map having a different FCF than the Stokes $Q$ and $U$ maps because of the attenuation caused by POL-2 and differences in the map-making procedure (cf. Friberg et al. 2016).

\section{Results}

\subsection{POL-2 Data Verification}

The BISTRO survey has recently begun to systematically investigate magnetic field structures in the dense cores using measurements of polarized dust emission, which is one of the most effective ways of probing the magnetic fields of such cores. Since POL-2 is newly commissioned, it is an important step to verify the consistency of our new data with those of previous studies. Therefore, we compare the POL-2 observations of $\rho$ Oph-A with data from the SCUPOL polarimeter on the previous-generation submillimeter bolometer array on the JCMT, SCUBA (Greaves et al. 1999).

Figure 1 shows the $850 \mu \mathrm{m}$ intensity map (Stokes $I$ ) obtained using the JCMT with SCUBA-2/POL-2, with well-known submillimeter and infrared sources labeled. The Stokes $I$ image 
(a)

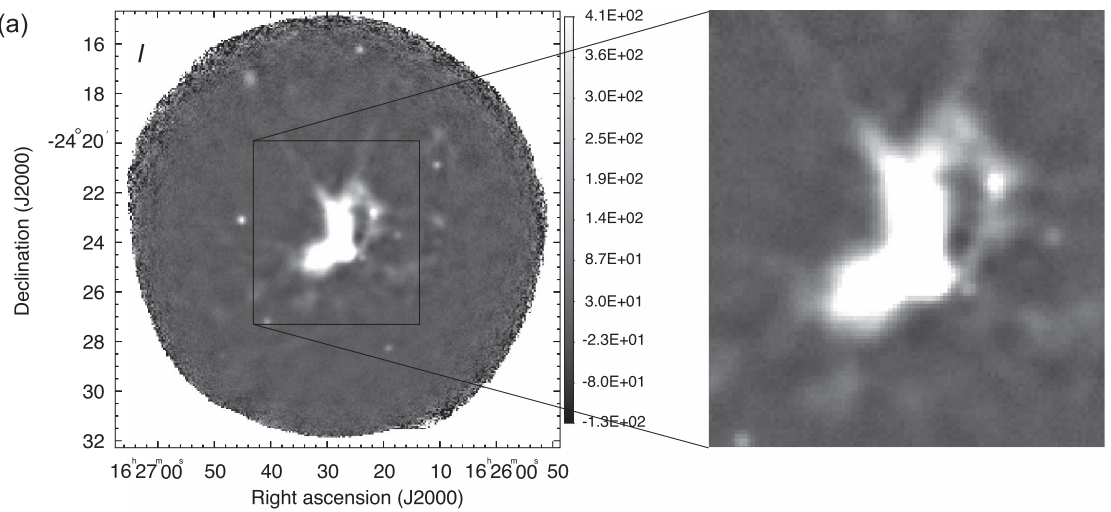

(b)
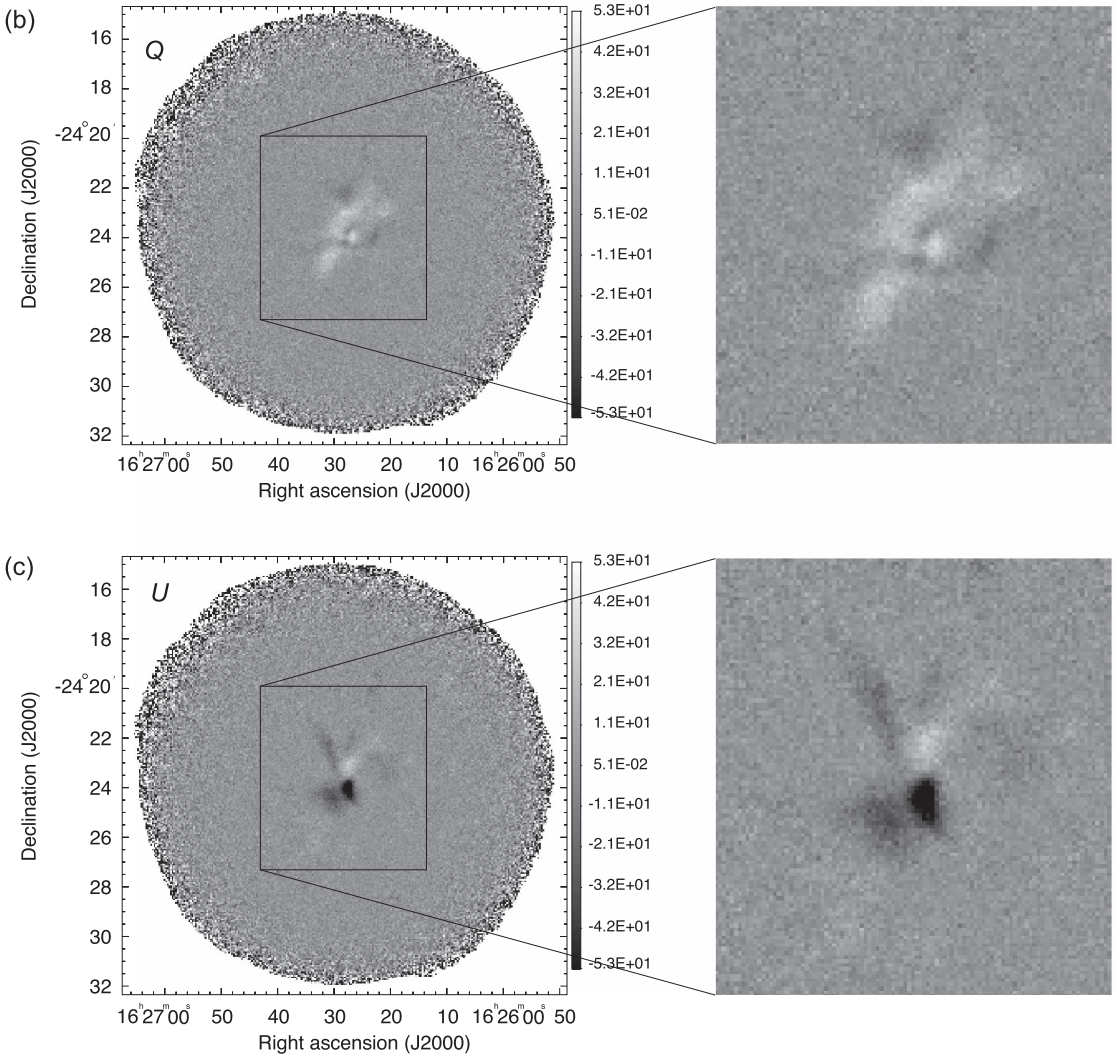
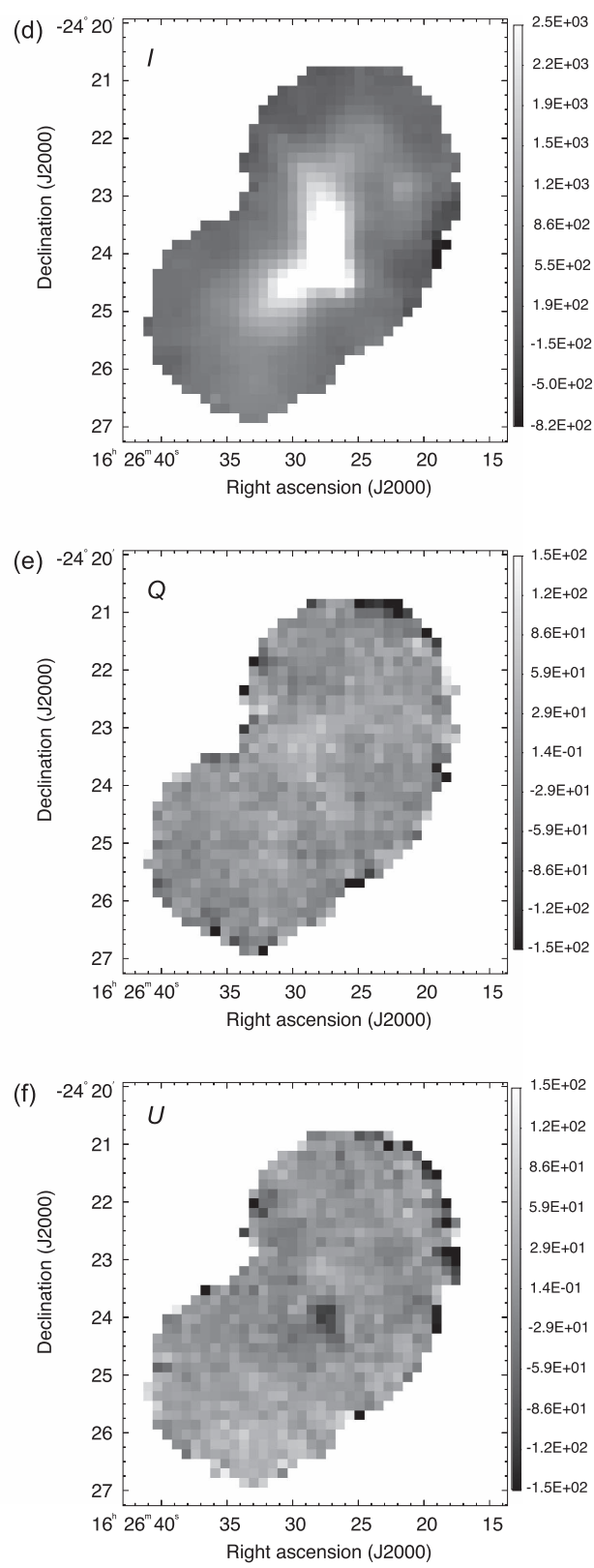

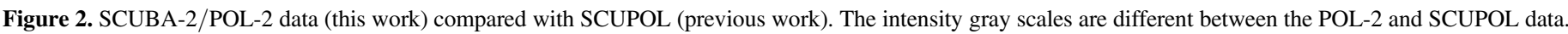

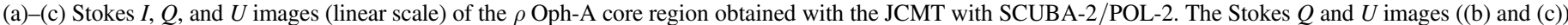

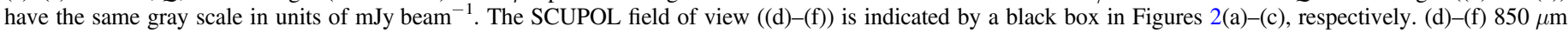

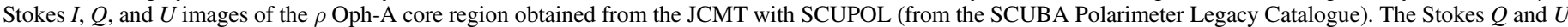

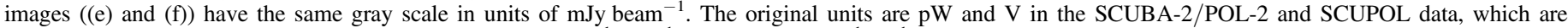

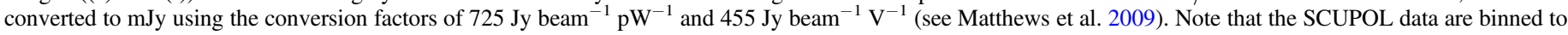

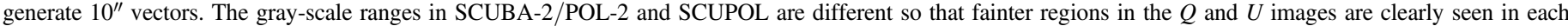
image.

is consistent with previous deep submillimeter continuum images (e.g., Pattle et al. 2015).

Figure 2 shows a comparison between the SCUBA-2/POL-2 data and the previous polarization data from SCUPOL (Greaves et al. 1999). Figures 2(a)-(c), respectively, show Stokes $I, Q$, and $U$ images of the $\rho$ Oph-A core region obtained from the JCMT with SCUBA-2/POL-2 (this work), and Figures 2(d)-(f), respectively, show Stokes $I, Q$, and $U$ images of the $\rho$ Oph-A core region obtained from the JCMT with SCUPOL (previous work; see also the SCUBA Polarimeter Legacy Catalogue; Matthews et al. 2009). The black boxes in Figures 2(a)-(c) show the regions covered by SCUPOL. As shown in Figure 2, our data are deeper and more clearly provide the morphology of the surrounding regions in all of the Stokes $I, Q$, and $U$ images, although two have the same spatial resolution. However, it should be noted that the SCUPOL data are binned to generate $10^{\prime \prime}$ polarization vectors. ${ }^{60}$

To compare the best intensity morphology with that from the previous data, we first introduce the detailed submillimeter morphology of the $\rho$ Oph-A core and then present the polarimetric results.

\footnotetext{
$\overline{{ }^{60}}$ The polarization vectors are not true vectors, since they give an orientation, not a direction.
} 


\subsection{Morphology of $\rho O p h-A$}

Figure 1 shows the morphology of $\rho$ Oph-A from our $850 \mu \mathrm{m}$ Stokes $I$ emission map. The region contains several subcores, which we outline below.

Oph-A SM1. Oph-A SM1 is a subcore located toward the peak of the $850 \mu \mathrm{m}$ intensity (Ward-Thompson et al. 1989; also cf. Figure 1). It has the brightest submillimeter continuum in all of $\rho$ Oph. The filamentary morphology in $\rho$ Oph suggests that SM1 may be influenced by the B4 star Oph S1 (cf. Figure 1), which is a nearby young B-type star. Motte et al. (1998) reported that the total mass and dust temperature of OphA SM1 are $2 M_{\odot}$ and $T \approx 20 \mathrm{~K}$, respectively.

VLA 1623. VLA 1623 is the prototypical Class 0 star (Andre et al. 1993). It drives a large-scale bipolar molecular outflow (Dent et al. 1995; Yu \& Chernin 1997) and is embedded within a nearly spherical dust envelope (Andre et al. 1993). Bontemps \& Andre (1997) found three emission clumps at centimeter wavelengths with the Very Large Array, which they interpreted as knots in the radio jet driving the large $\mathrm{CO}$ outflow (see also Chen et al. 2013). However, the position angles of the radio jet and the $\mathrm{CO}$ outflow differ by approximately $30^{\circ}$. Clump A was further resolved into two components at a high angular resolution (Chen et al. 2013) with the Submillimeter Array (SMA). VLA 1623 is also a binary system, with two components separated at high angular resolutions (Looney et al. 2000; Ward-Thompson et al. 2011). Since the POL-2 resolution is approximately 14 !. 1 at $850 \mu \mathrm{m}$, we cannot separate these components, and we refer to them as a single source, VLA 1623, in this paper.

Other local structures. There are two filaments in the northern part of the $\rho$ Oph-A core. These structures are consistent with not only the results obtained with SCUBA on the JCMT (Wilson et al. 1999) but also those seen in the map made with SCUBA-2 (Pattle et al. 2015) and IRAM (Motte et al. 1998) results. In addition to the filaments, Wilson et al. (1999) reported that there are two arcs of emission in the direction of the northwest extension of the VLA 1623 outflow. The outer arc appears relatively smooth at $850 \mu \mathrm{m}$, while the inner arc breaks up into a number of individual clumps, some of which are known protostars.

\subsection{New Submillimeter Polarization Vector Map}

Polarized thermal emission from dust grains in clouds offers an ideal probe of the magnetic field structure on multiple scales, from protostellar disks to cores and clumps (e.g., Matthews et al. 2001; Crutcher et al. 2004).

Figure 3 shows our submillimeter polarization vector maps of the $\rho$ Oph-A region observed with SCUBA-2/POL-2. Since it is worthwhile to directly compare our data with the previous submillimeter polarization vector map, Figure 3 is prepared with the same criteria, $I>0, P / \delta P>2$, and $\delta P<4 \%$, as were used in the previous results by SCUPOL (see Figure 44 of Matthews et al. 2009). The selection criteria here are mainly for the purpose of the comparison with the SCUPOL data; however, we have found this to be fairly reasonable to see the magnetic field structure in this region by changing various $P / \delta P$ or $\delta P$ selections. In addition, Figure 3 suggests that the vector maps with both $P / \delta P>2$ and $P / \delta P>3$ are almost the same, if we use the additional criterion of $\delta P<4 \%$. Without this $\delta P$ criterion, the vector map with $P / \delta P>2$ has many more vectors but also has rms noise values of $\delta P$ and $\delta \theta$ too high to allow interpretation of the magnetic field behavior. Therefore, we use the criteria of $I>0, P / \delta P>2$, and $\delta P<4 \%$ in the following discussion to maximize the number of polarization vectors that can be used for our discussion below on the magnetic field directions. The angle errors $(\delta \theta)$ of approximately $15^{\circ}$ in the $2 \sigma$ case are acceptable for such discussions. Therefore, we show both the $2 \sigma$ and $3 \sigma$ cases in several figures and use $2 \sigma$ data for the discussion on magnetic field directions.

Our data are more sensitive than those obtained by SCUPOL, as shown in Section 4.1. The new submillimeter polarization vectors inside the dense regions agree well with the results by Matthews et al. (2009), especially in the bright region near SM1. The dominant submillimeter polarization position angle in the bright region is approximately $130^{\circ}$ (as discussed in the following section). We have also checked whether our new data are consistent with the JCMT $800 \mu \mathrm{m}$ aperture polarimetric data of Holland et al. (1996). The measured positions are not exactly the same, but both the $P$ and $\theta$ values are consistent with each other between the two studies.

Note that there are clear inconsistencies between the SCUBA-2/POL-2 and SCUPOL data in the outer parts of the Oph-A core region. To the southeast and south of the core, the SCUPOL data show more numerous polarization vectors, even at a very low intensity level, while to the northwest and northeast, the SCUBA-2/POL-2 data reveal more vectors. Since our data have a higher $\mathrm{S} / \mathrm{N}$, as shown in Figure 2, we believe that our SCUBA-2/POL-2 data are more reliable in the faint outer regions down to approximately $30 \mathrm{mJy}_{\text {beam }}^{-1}$, while care is necessary when using the SCUPOL vectors in the outer regions. The reason why the SCUPOL data have more vectors in some outer core regions is not clear. However, we note that the SCUPOL maps were made by chopping, while POL-2 was in a scanning mode. Therefore, the chopping effect cannot be excluded in the SCUPOL data, which were taken at different times by different observers.

Based on the robustness toward the fainter regions mentioned above, our data clearly show the polarizations in the fainter regions surrounding the core, and the degrees of polarization are much higher ( $>5 \%)$ in the outer envelope. This trend is clear in our polarization map, whose vector length is proportional to the degree of polarization (see also Figure 7).

\subsection{Experimental Criteria}

Figure 4 shows the degree of polarization errors $(\delta P)$ versus the inverse intensity $\left(I^{-1}\right)$; the polarization uncertainty increases steadily with decreasing intensity. For $\delta P>4 \%$, we see significant scatter in this relation, whereas the data with $\delta P<4 \%$ are fairly well correlated. There are five vectors with $\delta P<4 \%$ that show substantial scatter from the main trend and are labeled in Figure 4 (cf. Table 1). Aside from these five cases, vectors with $\delta P<4 \%$ appear to be robust. All five anomalous positions (ID: 164, 237, 238, 239, 240) are located near the map boundary where the noise levels are higher. Vector 164 is located in the east of Oph-A, vector 237 is located between the northwest filamentary structure and GSS 26 , and vectors, 238, 239, and 240 are located in the upper part of the northeast filamentary structure. Note that including these five vectors does not affect our results. Figure 4 also shows that our polarization data present a large scatter when the intensity levels are less than approximately $30 \mathrm{mJy}^{\text {beam }}{ }^{-1}$, which 


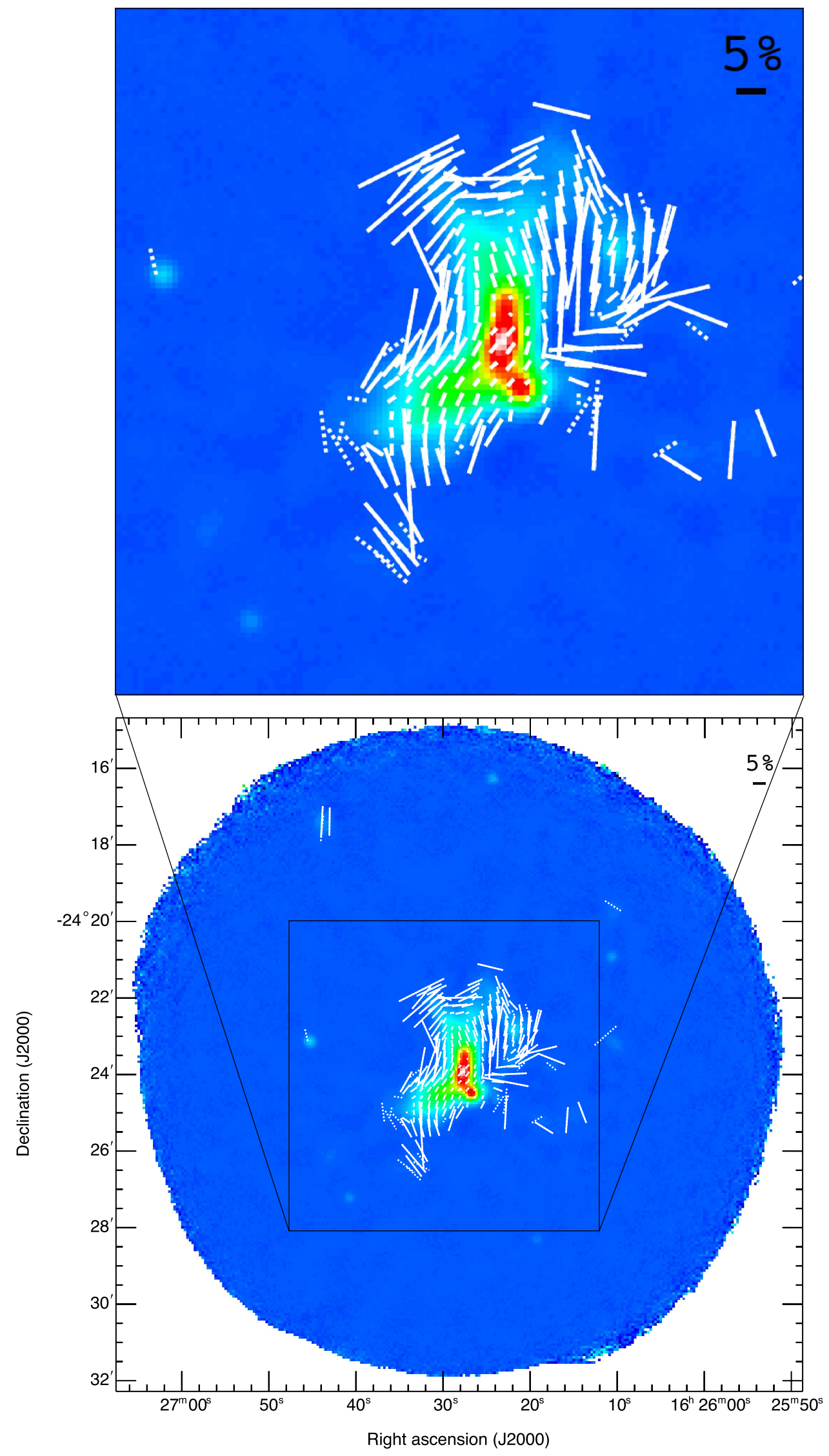

Figure 3. The $850 \mu \mathrm{m}$ polarization vector maps. The vectors are sampled on a $12^{\prime \prime}$ grid $(3 \times 3$ pixel smoothing) and plotted where $I>0, P / \delta P>2$, and $\delta P<4 \%$ (dotted vectors) and $I>0, P / \delta P>3$, and $\delta P<4 \%$ (solid vectors). 
(a)

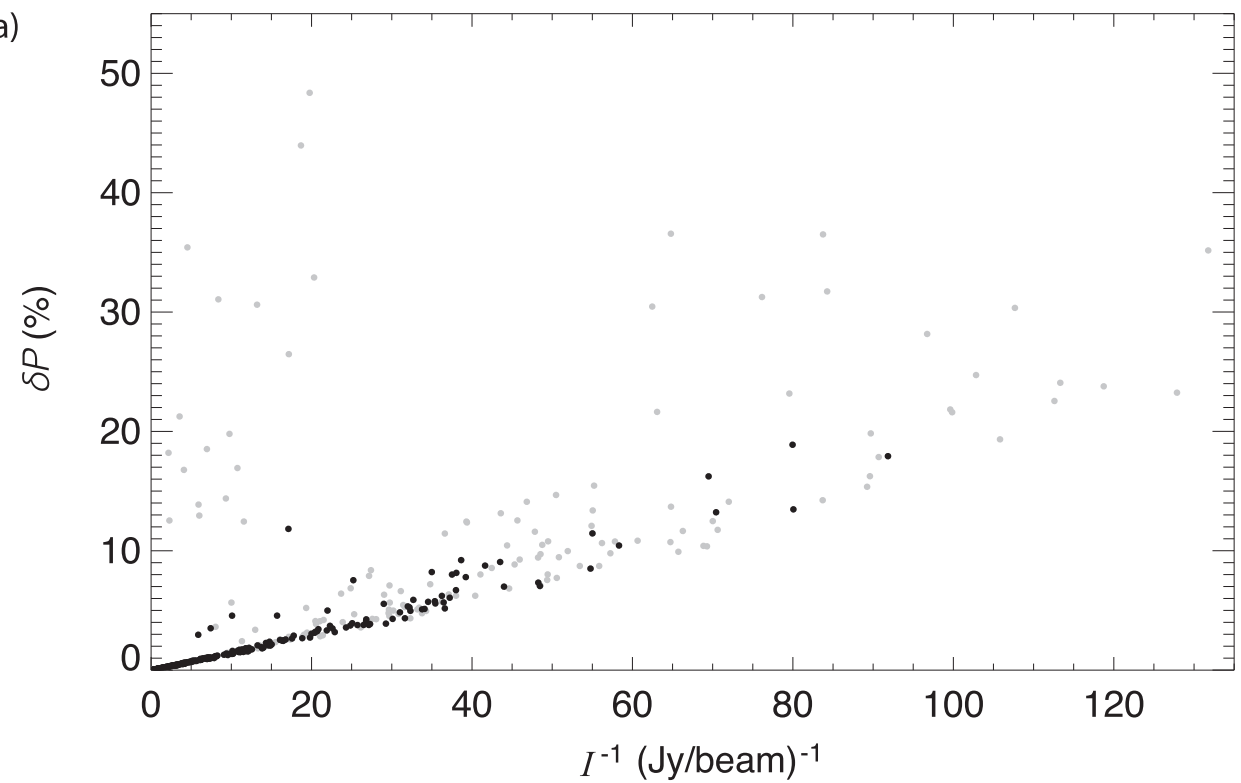

(b)

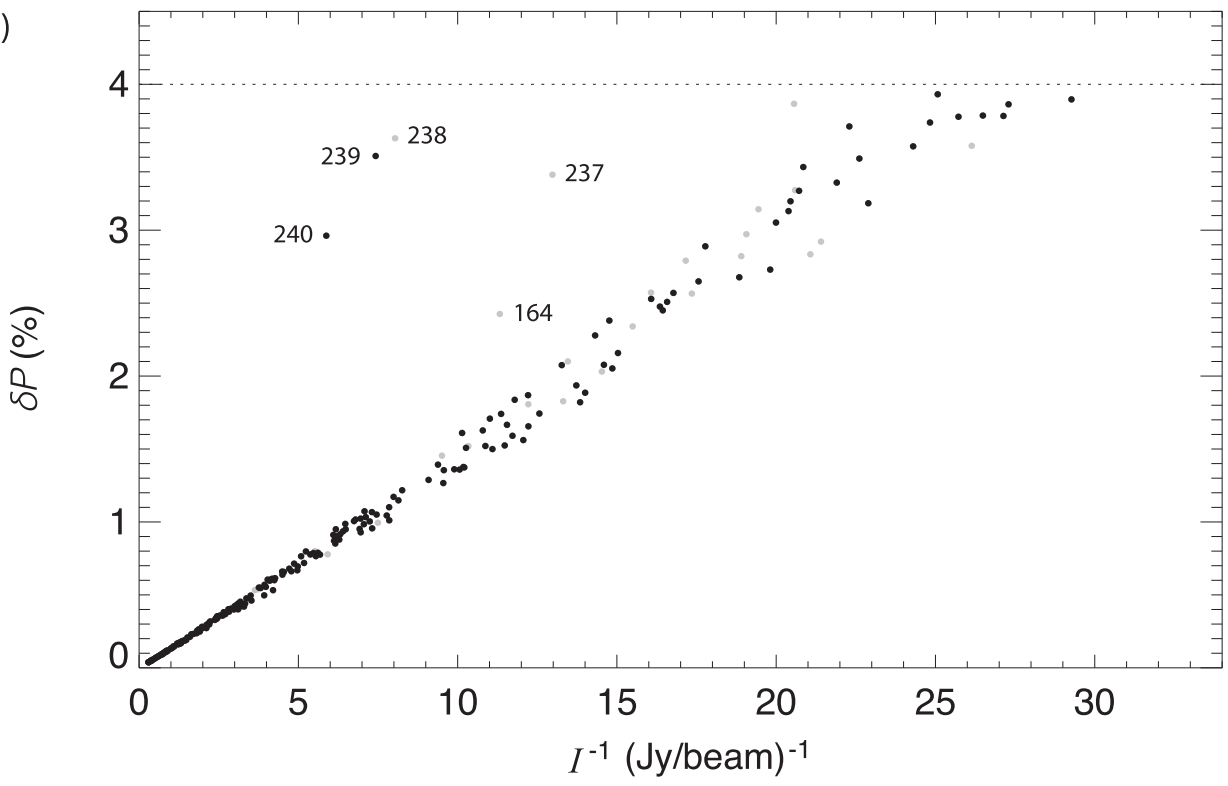

Figure 4. Degree of polarization errors $(\delta P)$ vs. 1 /intensity (I). Five dots with large $\delta P$ are labeled (cf. Table 1). These five sources are located in the outer regions of the Oph-A core region where the noise levels are higher. (a) Gray circles: $I>0$ and $P / \delta P>2$. Black circles: $I>0$ and $P / \delta P>3$. (b) Gray circles: $I>0, P / \delta P>2$, and $\delta P<4 \%$. Black circles: $I>0, P / \delta P>3$, and $\delta P<4 \%$.

corresponds to $N\left(\mathrm{H}_{2}\right) \sim 4 \times 10^{21} \mathrm{~cm}^{-2}$, assuming a temperature of $10 \mathrm{~K}$ (Kauffmann 2007).

\section{Discussion}

Magnetic fields in star formation are significant, as they can influence core collapse, star formation rates, and molecular cloud lifetimes (e.g., Myers \& Goodman 1988; Elmegreen 2000; Hartmann et al. 2001). We use our polarization data to determine the magnetic field strength in $\rho$ Oph-A below.

\subsection{Magnetic Field Structures in $\rho$ Oph-A}

The $\rho$ Oph molecular cloud has been observed with $1.3 \mathrm{~mm}$ continuum mapping (Motte et al. 1998) and line mapping (Umemoto et al. 1999; see also White et al. 2015), and the $\rho$ Oph-A core region is one of the most obvious sources.
Matthews et al. (2009) presented a bulk analysis of SCUPOL $850 \mu \mathrm{m}$ polarization vector maps, which include the $\rho$ Oph-A core. The submillimeter polarization position angle is about $130^{\circ}$, on average (measured east of north), which indicates a magnetic field direction of approximately $40^{\circ}$ (by rotating the submillimeter polarization vectors by $90^{\circ}$ ). This angle is consistent with the well-known $50^{\circ}$ component determined via infrared polarimetry observations (Sato et al. 1988; Kwon et al. 2015). Therefore, the magnetic field seems largely consistent between the outer low-density cloud and the high-density cores.

To investigate magnetic field structures in this region in more detail, we use the POL-2 polarization vectors rotated by $90^{\circ}$, as shown in Figure 5. Figure 6 shows the inferred morphology of the magnetic field in the $\rho$ Oph-A core region. In this figure, the vector maps are shown in two ways: one 
Table 1

Submillimeter Polarimetry in the $\rho$ Oph Cloud Core

\begin{tabular}{|c|c|c|c|c|c|c|c|c|}
\hline \multirow{2}{*}{ ID } & \multicolumn{2}{|c|}{ Position } & \multirow{2}{*}{$\begin{array}{c}I \pm \delta I \\
\left(\mathrm{mJy} \mathrm{beam}^{-1}\right)\end{array}$} & \multirow{2}{*}{$\begin{array}{c}Q \pm \delta Q \\
\left(\mathrm{mJy}_{\text {beam }}^{-1}\right)\end{array}$} & \multirow{2}{*}{$\begin{array}{c}U \pm \delta U \\
\left(\mathrm{mJy} \text { beam }^{-1}\right)\end{array}$} & \multirow{2}{*}{$\begin{array}{c}P \pm \delta P \\
(\%)\end{array}$} & \multirow{2}{*}{$\begin{array}{c}\theta \pm \delta \theta \\
(\operatorname{deg})\end{array}$} & \multirow{2}{*}{ Component } \\
\hline & $\alpha_{\mathbf{J} 2000}$ & $\delta_{\mathbf{J} 2000}$ & & & & & & \\
\hline 1 & $16: 26: 33.4$ & $-24: 26: 35.10$ & $51.430 \pm 2.098$ & $-0.342 \pm 1.619$ & $4.101 \pm 1.608$ & $7.37 \pm 3.14$ & $47.4 \pm 11.3$ & $\bar{j}$ \\
\hline 2 & $16: 26: 34.3$ & $-24: 26: 23.10$ & $58.272 \pm 2.122$ & $-1.394 \pm 1.615$ & $4.885 \pm 1.616$ & $8.26 \pm 2.79$ & $53.0 \pm 9.1$ & $\mathrm{j}$ \\
\hline 3 & $16: 26: 32.5$ & $-24: 26: 23.10$ & $62.223 \pm 2.095$ & $-2.642 \pm 1.595$ & $3.811 \pm 1.592$ & $7.00 \pm 2.57$ & $62.4 \pm 9.8$ & $\mathrm{j}$ \\
\hline 4 & $16: 26: 33.4$ & $-24: 26: 23.10$ & $67.742 \pm 2.103$ & $2.010 \pm 1.603$ & $6.867 \pm 1.597$ & $10.30 \pm 2.38$ & $36.8 \pm 6.4$ & $\mathrm{j}$ \\
\hline 5 & $16: 26: 33.4$ & $-24: 26: 11.10$ & $69.851 \pm 2.038$ & $1.644 \pm 1.576$ & $10.126 \pm 1.563$ & $14.51 \pm 2.28$ & $40.4 \pm 4.4$ & $\mathrm{j}$ \\
\hline 6 & $16: 26: 32.5$ & $-24: 26: 11.10$ & $74.294 \pm 2.043$ & $1.045 \pm 1.552$ & $4.014 \pm 1.556$ & $5.18 \pm 2.10$ & $37.7 \pm 10.7$ & $\mathrm{j}$ \\
\hline 7 & $16: 26: 32.5$ & $-24: 25: 59.10$ & $50.015 \pm 2.039$ & $2.705 \pm 1.519$ & $4.317 \pm 1.510$ & $9.73 \pm 3.05$ & $29.0 \pm 8.5$ & $\mathrm{j}$ \\
\hline 8 & $16: 26: 32.5$ & $-24: 25: 35.10$ & $39.887 \pm 2.062$ & $10.629 \pm 1.467$ & $1.343 \pm 1.482$ & $26.61 \pm 3.93$ & $3.6 \pm 4.0$ & $\mathrm{j}$ \\
\hline 9 & $16: 26: 34.3$ & $-24: 25: 23.10$ & $61.159 \pm 2.082$ & $2.735 \pm 1.496$ & $4.664 \pm 1.506$ & $8.49 \pm 2.48$ & $29.8 \pm 7.9$ & $\mathrm{j}$ \\
\hline 10 & $16: 26: 33.4$ & $-24: 25: 23.10$ & $106.577 \pm 2.065$ & $5.813 \pm 1.473$ & $4.804 \pm 1.484$ & $6.94 \pm 1.39$ & $19.8 \pm 5.6$ & $\mathrm{j}$ \\
\hline 11 & $16: 26: 32.5$ & $-24: 25: 23.10$ & $136.809 \pm 2.039$ & $9.366 \pm 1.450$ & $5.233 \pm 1.458$ & $7.77 \pm 1.07$ & $14.6 \pm 3.9$ & $\mathrm{j}$ \\
\hline 12 & $16: 26: 30.8$ & $-24: 25: 23.10$ & $140.545 \pm 2.035$ & $7.720 \pm 1.442$ & $5.518 \pm 1.452$ & $6.67 \pm 1.03$ & $17.8 \pm 4.4$ & $\mathrm{j}$ \\
\hline 13 & $16: 26: 31.6$ & $-24: 25: 23.10$ & $158.406 \pm 2.045$ & $10.415 \pm 1.443$ & $3.233 \pm 1.449$ & $6.82 \pm 0.92$ & $8.6 \pm 3.8$ & $\mathrm{j}$ \\
\hline 14 & $16: 26: 18.5$ & $-24: 25: 23.08$ & $60.328 \pm 2.255$ & $-2.298 \pm 1.482$ & $4.702 \pm 1.505$ & $8.31 \pm 2.51$ & $58.0 \pm 8.1$ & $\ldots$ \\
\hline 15 & $16: 26: 29.0$ & $-24: 25: 11.10$ & $72.854 \pm 2.037$ & $5.357 \pm 1.399$ & $-3.862 \pm 1.396$ & $8.86 \pm 1.94$ & $-17.9 \pm 6.1$ & $\cdots$ \\
\hline 16 & $16: 26: 34.3$ & $-24: 25: 11.10$ & $163.985 \pm 2.057$ & $-2.271 \pm 1.473$ & $5.622 \pm 1.495$ & $3.58 \pm 0.91$ & $56.0 \pm 7.0$ & $\mathrm{i}$ \\
\hline 17 & $16: 26: 29.9$ & $-24: 25: 11.10$ & $179.956 \pm 2.042$ & $1.486 \pm 1.404$ & $-3.252 \pm 1.405$ & $1.83 \pm 0.78$ & $-32.7 \pm 11.3$ & $\mathrm{i}$ \\
\hline 18 & $16: 26: 33.4$ & $-24: 25: 11.10$ & $243.825 \pm 2.065$ & $5.194 \pm 1.455$ & $0.295 \pm 1.469$ & $2.05 \pm 0.60$ & $1.6 \pm 8.1$ & $\mathrm{i}$ \\
\hline 19 & $16: 26: 32.5$ & $-24: 25: 11.10$ & $263.398 \pm 2.060$ & $11.865 \pm 1.441$ & $0.168 \pm 1.450$ & $4.47 \pm 0.55$ & $0.4 \pm 3.5$ & $\mathrm{i}$ \\
\hline 20 & $16: 26: 31.6$ & $-24: 25: 11.10$ & $285.549 \pm 2.022$ & $15.906 \pm 1.409$ & $2.776 \pm 1.435$ & $5.63 \pm 0.50$ & $5.0 \pm 2.5$ & $\mathrm{i}$ \\
\hline 21 & $16: 26: 30.8$ & $-24: 25: 11.10$ & $296.876 \pm 2.016$ & $16.204 \pm 1.409$ & $1.031 \pm 1.408$ & $5.45 \pm 0.48$ & $1.8 \pm 2.5$ & $\mathrm{i}$ \\
\hline 22 & $16: 26: 36.0$ & $-24: 25: 11.09$ & $52.454 \pm 2.123$ & $2.267 \pm 1.542$ & $4.078 \pm 1.549$ & $8.39 \pm 2.97$ & $30.5 \pm 9.5$ & $\cdots$ \\
\hline 23 & $16: 26: 35.2$ & $-24: 25: 11.09$ & $64.541 \pm 2.038$ & $0.240 \pm 1.505$ & $4.359 \pm 1.504$ & $6.35 \pm 2.34$ & $43.4 \pm 9.9$ & $\ldots$ \\
\hline 24 & $16: 26: 19.3$ & $-24: 25: 11.08$ & $57.627 \pm 2.173$ & $-1.546 \pm 1.457$ & $-2.992 \pm 1.477$ & $5.26 \pm 2.57$ & $-58.7 \pm 12.4$ & $\cdots$ \\
\hline 25 & $16: 26: 15.8$ & $-24: 25: 11.06$ & $48.901 \pm 2.371$ & $5.178 \pm 1.543$ & $-0.781 \pm 1.566$ & $10.23 \pm 3.20$ & $-4.3 \pm 8.6$ & $\cdots$ \\
\hline 26 & $16: 26: 28.1$ & $-24: 24: 59.10$ & $79.582 \pm 2.088$ & $2.094 \pm 1.378$ & $-4.110 \pm 1.383$ & $5.53 \pm 1.74$ & $-31.5 \pm 8.6$ & $\ldots$ \\
\hline 27 & $16: 26: 29.0$ & $-24: 24: 59.10$ & $209.209 \pm 2.086$ & $7.624 \pm 1.374$ & $-7.347 \pm 1.388$ & $5.02 \pm 0.66$ & $-22.0 \pm 3.7$ & $\cdots$ \\
\hline 28 & $16: 26: 34.3$ & $-24: 24: 59.10$ & $275.086 \pm 2.023$ & $-3.445 \pm 1.464$ & $-0.868 \pm 1.472$ & $1.18 \pm 0.53$ & $-82.9 \pm 11.9$ & $\mathrm{i}$ \\
\hline 29 & $16: 26: 33.4$ & $-24: 24: 59.10$ & $407.349 \pm 2.051$ & $7.166 \pm 1.441$ & $2.139 \pm 1.454$ & $1.80 \pm 0.35$ & $8.3 \pm 5.6$ & $\mathrm{i}$ \\
\hline 30 & $16: 26: 29.9$ & $-24: 24: 59.10$ & $421.360 \pm 2.050$ & $9.406 \pm 1.387$ & $-10.463 \pm 1.378$ & $3.32 \pm 0.33$ & $-24.0 \pm 2.8$ & $\mathrm{i}$ \\
\hline 31 & $16: 26: 30.8$ & $-24: 24: 59.10$ & $472.817 \pm 2.054$ & $19.633 \pm 1.387$ & $-6.862 \pm 1.412$ & $4.39 \pm 0.29$ & $-9.6 \pm 1.9$ & $\mathrm{i}$ \\
\hline 32 & $16: 26: 32.5$ & $-24: 24: 59.10$ & $506.147 \pm 2.067$ & $14.517 \pm 1.422$ & $-3.031 \pm 1.424$ & $2.92 \pm 0.28$ & $-5.9 \pm 2.8$ & $\mathrm{i}$ \\
\hline 33 & $16: 26: 31.6$ & $-24: 24: 59.10$ & $535.088 \pm 2.043$ & $20.017 \pm 1.408$ & $-4.015 \pm 1.409$ & $3.81 \pm 0.26$ & $-5.7 \pm 2.0$ & $\mathrm{i}$ \\
\hline 34 & $16: 26: 22.9$ & $-24: 24: 59.09$ & $36.637 \pm 2.072$ & $4.997 \pm 1.386$ & $-0.534 \pm 1.386$ & $13.19 \pm 3.86$ & $-3.1 \pm 7.9$ & $\mathrm{~h}$ \\
\hline 35 & $16: 26: 36.9$ & $-24: 24: 59.09$ & $48.555 \pm 2.180$ & $3.892 \pm 1.580$ & $0.981 \pm 1.573$ & $7.60 \pm 3.27$ & $7.1 \pm 11.2$ & $\cdots$ \\
\hline 36 & $16: 26: 36.0$ & $-24: 24: 59.09$ & $105.173 \pm 2.056$ & $4.079 \pm 1.527$ & $-1.656 \pm 1.529$ & $3.93 \pm 1.45$ & $-11.0 \pm 9.9$ & $\cdots$ \\
\hline 37 & $16: 26: 14.1$ & $-24: 24: 59.05$ & $56.258 \pm 2.532$ & $3.874 \pm 1.604$ & $3.473 \pm 1.615$ & $8.80 \pm 2.89$ & $20.9 \pm 8.9$ & $\cdots$ \\
\hline 38 & $16: 26: 25.5$ & $-24: 24: 47.10$ & $128.626 \pm 2.111$ & $2.576 \pm 1.331$ & $-3.453 \pm 1.347$ & $3.18 \pm 1.04$ & $-26.6 \pm 8.9$ & $\mathrm{~h}$ \\
\hline 39 & $16: 26: 27.3$ & $-24: 24: 47.10$ & $315.284 \pm 2.174$ & $6.195 \pm 1.357$ & $-1.949 \pm 1.352$ & $2.01 \pm 0.43$ & $-8.7 \pm 6.0$ & $\cdots$ \\
\hline 40 & $16: 26: 28.1$ & $-24: 24: 47.10$ & $322.238 \pm 2.094$ & $3.581 \pm 1.354$ & $-9.384 \pm 1.351$ & $3.09 \pm 0.42$ & $-34.6 \pm 3.9$ & $\ldots$ \\
\hline 41 & $16: 26: 33.4$ & $-24: 24: 47.10$ & $448.077 \pm 2.046$ & $6.608 \pm 1.425$ & $-0.974 \pm 1.431$ & $1.46 \pm 0.32$ & $-4.2 \pm 6.1$ & $\mathrm{i}$ \\
\hline 42 & $16: 26: 29.0$ & $-24: 24: 47.10$ & $505.237 \pm 2.110$ & $9.237 \pm 1.360$ & $-16.107 \pm 1.369$ & $3.67 \pm 0.27$ & $-30.1 \pm 2.1$ & $\cdots$ \\
\hline 43 & $16: 26: 32.5$ & $-24: 24: 47.10$ & $608.888 \pm 2.048$ & $12.679 \pm 1.403$ & $-2.942 \pm 1.413$ & $2.13 \pm 0.23$ & $-6.5 \pm 3.1$ & $\mathrm{i}$ \\
\hline 44 & $16: 26: 29.9$ & $-24: 24: 47.10$ & $781.876 \pm 2.061$ & $20.867 \pm 1.368$ & $-17.607 \pm 1.377$ & $3.49 \pm 0.18$ & $-20.1 \pm 1.4$ & $\mathrm{i}$ \\
\hline 45 & $16: 26: 31.6$ & $-24: 24: 47.10$ & $834.935 \pm 2.056$ & $24.301 \pm 1.389$ & $-9.222 \pm 1.397$ & $3.11 \pm 0.17$ & $-10.4 \pm 1.5$ & $\mathrm{i}$ \\
\hline 46 & $16: 26: 30.8$ & $-24: 24: 47.10$ & $834.771 \pm 2.034$ & $21.231 \pm 1.376$ & $-13.423 \pm 1.377$ & $3.00 \pm 0.17$ & $-16.2 \pm 1.6$ & $\mathrm{i}$ \\
\hline 47 & $16: 26: 22.9$ & $-24: 24: 47.09$ & $38.256 \pm 2.075$ & $2.747 \pm 1.353$ & $-1.657 \pm 1.371$ & $7.60 \pm 3.58$ & $-15.6 \pm 12.2$ & $\mathrm{~h}$ \\
\hline 48 & $16: 26: 23.7$ & $-24: 24: 47.09$ & $46.716 \pm 2.083$ & $1.630 \pm 1.347$ & $-3.585 \pm 1.355$ & $7.92 \pm 2.92$ & $-32.8 \pm 9.8$ & $\mathrm{~h}$ \\
\hline 49 & $16: 26: 33.4$ & $-24: 24: 35.10$ & $375.056 \pm 2.013$ & $-1.943 \pm 1.399$ & $-5.926 \pm 1.412$ & $1.62 \pm 0.38$ & $-54.1 \pm 6.4$ & $\mathrm{i}$ \\
\hline 50 & $16: 26: 32.5$ & $-24: 24: 35.10$ & $550.300 \pm 2.058$ & $3.323 \pm 1.387$ & $-6.657 \pm 1.393$ & $1.33 \pm 0.25$ & $-31.7 \pm 5.3$ & $\mathrm{i}$ \\
\hline 51 & $16: 26: 25.5$ & $-24: 24: 35.10$ & $553.396 \pm 2.136$ & $5.956 \pm 1.308$ & $-5.067 \pm 1.317$ & $1.39 \pm 0.24$ & $-20.2 \pm 4.8$ & $\mathrm{~h}$ \\
\hline 52 & $16: 26: 31.6$ & $-24: 24: 35.10$ & $930.155 \pm 2.048$ & $16.612 \pm 1.368$ & $-14.349 \pm 1.370$ & $2.36 \pm 0.15$ & $-20.4 \pm 1.8$ & $\mathrm{i}$ \\
\hline 53 & $16: 26: 28.1$ & $-24: 24: 35.10$ & $1003.480 \pm 2.149$ & $9.105 \pm 1.330$ & $-12.010 \pm 1.332$ & $1.50 \pm 0.13$ & $-26.4 \pm 2.5$ & $\mathrm{a}$ \\
\hline 54 & $16: 26: 29.0$ & $-24: 24: 35.10$ & $1119.110 \pm 2.061$ & $17.792 \pm 1.332$ & $-14.137 \pm 1.336$ & $2.03 \pm 0.12$ & $-19.2 \pm 1.7$ & a \\
\hline 55 & $16: 26: 30.8$ & $-24: 24: 35.10$ & $1174.260 \pm 2.021$ & $20.439 \pm 1.352$ & $-20.801 \pm 1.362$ & $2.48 \pm 0.12$ & $-22.8 \pm 1.3$ & $\mathrm{i}$ \\
\hline 56 & $16: 26: 29.9$ & $-24: 24: 35.10$ & $1282.560 \pm 2.076$ & $19.135 \pm 1.338$ & $-24.433 \pm 1.349$ & $2.42 \pm 0.10$ & $-26.0 \pm 1.2$ & $\mathrm{i}$ \\
\hline 57 & $16: 26: 27.3$ & $-24: 24: 35.10$ & $1371.780 \pm 2.362$ & $7.661 \pm 1.329$ & $-11.563 \pm 1.319$ & $1.01 \pm 0.10$ & $-28.2 \pm 2.7$ & a \\
\hline 58 & $16: 26: 26.4$ & $-24: 24: 35.10$ & $1887.860 \pm 2.594$ & $3.423 \pm 1.319$ & $-17.786 \pm 1.322$ & $0.96 \pm 0.07$ & $-39.6 \pm 2.1$ & $\mathrm{a}$ \\
\hline 59 & $16: 26: 22.9$ & $-24: 24: 35.09$ & $47.454 \pm 2.053$ & $3.372 \pm 1.335$ & $-0.894 \pm 1.355$ & $6.79 \pm 2.83$ & $-7.4 \pm 11.1$ & $\mathrm{~h}$ \\
\hline 60 & $16: 26: 35.2$ & $-24: 24: 35.09$ & $96.748 \pm 2.068$ & $-3.614 \pm 1.467$ & $1.021 \pm 1.481$ & $3.57 \pm 1.52$ & $82.1 \pm 11.3$ & $\cdots$ \\
\hline 61 & $16: 26: 23.7$ & $-24: 24: 35.09$ & $168.924 \pm 2.043$ & $3.591 \pm 1.313$ & $0.056 \pm 1.332$ & $1.98 \pm 0.78$ & $0.4 \pm 10.6$ & $\mathrm{~h}$ \\
\hline 62 & $16: 26: 34.3$ & $-24: 24: 23.10$ & $162.147 \pm 2.032$ & $1.783 \pm 1.437$ & $-7.161 \pm 1.433$ & $4.46 \pm 0.89$ & $-38.0 \pm 5.6$ & i \\
\hline 63 & $16: 26: 33.4$ & $-24: 24: 23.10$ & $333.190 \pm 2.020$ & $-2.890 \pm 1.380$ & $-10.016 \pm 1.409$ & $3.10 \pm 0.42$ & $-53.0 \pm 3.8$ & $\mathrm{i}$ \\
\hline
\end{tabular}


Table 1

(Continued)

\begin{tabular}{|c|c|c|c|c|c|c|c|c|}
\hline \multirow{2}{*}{ ID } & \multicolumn{2}{|c|}{ Position } & \multirow{2}{*}{$\begin{array}{c}I \pm \delta I \\
\left(\mathrm{mJy}^{-1} \text { beam }^{-1}\right)\end{array}$} & \multirow{2}{*}{$\begin{array}{c}Q \pm \delta Q \\
\left(\mathrm{mJy}^{\text {beam }}{ }^{-1}\right)\end{array}$} & \multirow{2}{*}{$\begin{array}{c}U \pm \delta U \\
\left(\mathrm{mJy}^{-1} \text { beam }^{-1}\right)\end{array}$} & \multirow{2}{*}{$\begin{array}{c}P \pm \delta P \\
(\%)\end{array}$} & \multirow{2}{*}{$\begin{array}{c}\theta \pm \delta \theta \\
(\operatorname{deg})\end{array}$} & \multirow{2}{*}{ Component } \\
\hline & $\alpha_{\mathrm{J} 2000}$ & $\delta_{\mathrm{J} 2000}$ & & & & & & \\
\hline 64 & $16: 26: 32.5$ & $-24: 24: 23.10$ & $534.381 \pm 1.993$ & $1.523 \pm 1.365$ & $-8.430 \pm 1.379$ & $1.58 \pm 0.26$ & $-39.9 \pm 4.6$ & $\mathrm{i}$ \\
\hline 65 & $16: 26: 25.5$ & $-24: 24: 23.10$ & $778.337 \pm 2.115$ & $-0.120 \pm 1.295$ & $-5.106 \pm 1.291$ & $0.63 \pm 0.17$ & $-45.7 \pm 7.3$ & $\ldots$ \\
\hline 66 & $16: 26: 31.6$ & $-24: 24: 23.10$ & $816.746 \pm 2.022$ & $4.701 \pm 1.351$ & $-20.827 \pm 1.353$ & $2.61 \pm 0.17$ & $-38.6 \pm 1.8$ & i \\
\hline 67 & $16: 26: 30.8$ & $-24: 24: 23.10$ & $1158.130 \pm 1.998$ & $11.575 \pm 1.341$ & $-27.026 \pm 1.337$ & $2.54 \pm 0.12$ & $-33.4 \pm 1.3$ & $\mathrm{i}$ \\
\hline 68 & $16: 26: 29.9$ & $-24: 24: 23.10$ & $1493.050 \pm 2.017$ & $12.956 \pm 1.320$ & $-31.557 \pm 1.326$ & $2.28 \pm 0.09$ & $-33.8 \pm 1.1$ & $\mathrm{i}$ \\
\hline 69 & $16: 26: 29.0$ & $-24: 24: 23.10$ & $1660.180 \pm 2.071$ & $8.695 \pm 1.314$ & $-23.456 \pm 1.304$ & $1.50 \pm 0.08$ & $-34.8 \pm 1.5$ & $\mathrm{a}$ \\
\hline 70 & $16: 26: 28.1$ & $-24: 24: 23.10$ & $1964.770 \pm 2.153$ & $3.659 \pm 1.314$ & $-30.492 \pm 1.312$ & $1.56 \pm 0.07$ & $-41.6 \pm 1.2$ & $\mathrm{a}$ \\
\hline 71 & $16: 26: 26.4$ & $-24: 24: 23.10$ & $2416.880 \pm 2.535$ & $-4.410 \pm 1.289$ & $-25.770 \pm 1.303$ & $1.08 \pm 0.05$ & $-49.9 \pm 1.4$ & $\mathrm{a}$ \\
\hline 72 & $16: 26: 27.3$ & $-24: 24: 23.10$ & $2643.620 \pm 2.315$ & $4.500 \pm 1.290$ & $-42.859 \pm 1.297$ & $1.63 \pm 0.05$ & $-42.0 \pm 0.9$ & $\mathrm{a}$ \\
\hline 73 & $16: 26: 23.7$ & $-24: 24: 23.09$ & $136.669 \pm 2.065$ & $-3.840 \pm 1.302$ & $3.425 \pm 1.307$ & $3.64 \pm 0.96$ & $69.1 \pm 7.3$ & $\cdots$ \\
\hline 74 & $16: 26: 34.3$ & $-24: 24: 11.10$ & $86.585 \pm 2.059$ & $4.128 \pm 1.419$ & $-6.984 \pm 1.433$ & $9.22 \pm 1.67$ & $-29.7 \pm 5.0$ & $\mathrm{~g}$ \\
\hline 75 & $16: 26: 33.4$ & $-24: 24: 11.10$ & $200.755 \pm 2.019$ & $1.662 \pm 1.381$ & $-9.789 \pm 1.392$ & $4.90 \pm 0.70$ & $-40.2 \pm 4.0$ & $\mathrm{~g}$ \\
\hline 76 & $16: 26: 32.5$ & $-24: 24: 11.10$ & $354.117 \pm 1.999$ & $-0.931 \pm 1.355$ & $-17.182 \pm 1.359$ & $4.84 \pm 0.38$ & $-46.6 \pm 2.3$ & $\mathrm{~g}$ \\
\hline 77 & $16: 26: 31.6$ & $-24: 24: 11.10$ & $454.205 \pm 1.989$ & $3.053 \pm 1.323$ & $-22.731 \pm 1.347$ & $5.04 \pm 0.30$ & $-41.2 \pm 1.7$ & $\mathrm{~g}$ \\
\hline 78 & $16: 26: 25.5$ & $-24: 24: 11.10$ & $473.293 \pm 2.094$ & $1.082 \pm 1.276$ & $-8.387 \pm 1.288$ & $1.77 \pm 0.27$ & $-41.3 \pm 4.3$ & f \\
\hline 79 & $16: 26: 30.8$ & $-24: 24: 11.10$ & $627.260 \pm 2.010$ & $3.335 \pm 1.322$ & $-28.680 \pm 1.329$ & $4.60 \pm 0.21$ & $-41.7 \pm 1.3$ & $\mathrm{~g}$ \\
\hline 80 & $16: 26: 29.9$ & $-24: 24: 11.10$ & $953.887 \pm 2.014$ & $9.991 \pm 1.303$ & $-26.395 \pm 1.314$ & $2.96 \pm 0.14$ & $-34.6 \pm 1.3$ & $\mathrm{~g}$ \\
\hline 81 & $16: 26: 26.4$ & $-24: 24: 11.10$ & $1417.450 \pm 2.182$ & $11.137 \pm 1.272$ & $-24.760 \pm 1.281$ & $1.91 \pm 0.09$ & $-32.9 \pm 1.3$ & $\mathrm{a}$ \\
\hline 82 & $16: 26: 29.0$ & $-24: 24: 11.10$ & $1576.900 \pm 2.115$ & $1.175 \pm 1.300$ & $-26.510 \pm 1.300$ & $1.68 \pm 0.08$ & $-43.7 \pm 1.4$ & $\mathrm{a}$ \\
\hline 83 & $16: 26: 27.3$ & $-24: 24: 11.10$ & $2618.600 \pm 2.216$ & $6.129 \pm 1.275$ & $-57.370 \pm 1.284$ & $2.20 \pm 0.05$ & $-42.0 \pm 0.6$ & $\mathrm{a}$ \\
\hline 84 & $16: 26: 28.1$ & $-24: 24: 11.10$ & $2697.520 \pm 2.215$ & $-6.913 \pm 1.285$ & $-40.965 \pm 1.300$ & $1.54 \pm 0.05$ & $-49.8 \pm 0.9$ & $\mathrm{a}$ \\
\hline 85 & $16: 26: 22.9$ & $-24: 24: 11.09$ & $50.474 \pm 2.353$ & $-8.634 \pm 1.307$ & $3.476 \pm 1.310$ & $18.26 \pm 2.73$ & $79.0 \pm 4.0$ & $\mathrm{f}$ \\
\hline 86 & $16: 26: 23.7$ & $-24: 24: 11.09$ & $127.343 \pm 2.063$ & $-6.138 \pm 1.283$ & $1.183 \pm 1.297$ & $4.80 \pm 1.01$ & $84.5 \pm 5.9$ & $f$ \\
\hline 87 & $16: 26: 33.4$ & $-24: 23: 59.10$ & $68.829 \pm 2.028$ & $-1.088 \pm 1.388$ & $-3.856 \pm 1.393$ & $5.46 \pm 2.03$ & $-52.9 \pm 9.9$ & $\mathrm{~g}$ \\
\hline 88 & $16: 26: 32.5$ & $-24: 23: 59.10$ & $144.438 \pm 1.971$ & $3.840 \pm 1.353$ & $-11.073 \pm 1.367$ & $8.06 \pm 0.95$ & $-35.4 \pm 3.3$ & $\mathrm{~g}$ \\
\hline 89 & $16: 26: 31.6$ & $-24: 23: 59.10$ & $201.312 \pm 1.996$ & $7.689 \pm 1.324$ & $-18.711 \pm 1.332$ & $10.03 \pm 0.67$ & $-33.8 \pm 1.9$ & $\mathrm{~g}$ \\
\hline 90 & $16: 26: 25.5$ & $-24: 23: 59.10$ & $254.610 \pm 2.083$ & $9.190 \pm 1.261$ & $-3.073 \pm 1.279$ & $3.77 \pm 0.50$ & $-9.2 \pm 3.8$ & f \\
\hline 91 & $16: 26: 30.8$ & $-24: 23: 59.10$ & $300.531 \pm 2.007$ & $8.665 \pm 1.317$ & $-18.943 \pm 1.315$ & $6.92 \pm 0.44$ & $-32.7 \pm 1.8$ & g \\
\hline 92 & $16: 26: 29.9$ & $-24: 23: 59.10$ & $524.082 \pm 2.014$ & $10.789 \pm 1.291$ & $-21.277 \pm 1.305$ & $4.55 \pm 0.25$ & $-31.6 \pm 1.6$ & $\mathrm{~g}$ \\
\hline 93 & $16: 26: 29.0$ & $-24: 23: 59.10$ & $1115.710 \pm 2.140$ & $17.291 \pm 1.284$ & $-27.305 \pm 1.295$ & $2.89 \pm 0.12$ & $-28.8 \pm 1.1$ & $\mathrm{a}$ \\
\hline 94 & $16: 26: 26.4$ & $-24: 23: 59.10$ & $1292.280 \pm 2.185$ & $26.583 \pm 1.273$ & $-17.224 \pm 1.278$ & $2.45 \pm 0.10$ & $-16.5 \pm 1.2$ & $\mathrm{a}$ \\
\hline 95 & $16: 26: 27.3$ & $-24: 23: 59.10$ & $3352.910 \pm 2.478$ & $23.719 \pm 1.283$ & $-75.009 \pm 1.285$ & $2.35 \pm 0.04$ & $-36.2 \pm 0.5$ & $\mathrm{a}$ \\
\hline 96 & $16: 26: 28.1$ & $-24: 23: 59.10$ & $3402.040 \pm 2.498$ & $11.316 \pm 1.285$ & $-63.491 \pm 1.298$ & $1.90 \pm 0.04$ & $-39.9 \pm 0.6$ & $\mathrm{a}$ \\
\hline 97 & $16: 26: 22.9$ & $-24: 23: 59.09$ & $81.824 \pm 2.074$ & $-13.510 \pm 1.310$ & $-1.131 \pm 1.321$ & $16.49 \pm 1.66$ & $-87.6 \pm 2.8$ & $f$ \\
\hline 98 & $16: 26: 23.7$ & $-24: 23: 59.09$ & $82.906 \pm 2.072$ & $-4.200 \pm 1.289$ & $1.345 \pm 1.299$ & $5.09 \pm 1.56$ & $81.1 \pm 8.4$ & $f$ \\
\hline 99 & $16: 26: 32.5$ & $-24: 23: 47.10$ & $43.684 \pm 1.993$ & $6.401 \pm 1.355$ & $-2.398 \pm 1.360$ & $15.34 \pm 3.18$ & $-10.3 \pm 5.7$ & $\mathrm{~g}$ \\
\hline 100 & $16: 26: 31.6$ & $-24: 23: 47.10$ & $67.312 \pm 1.970$ & $9.691 \pm 1.331$ & $-7.843 \pm 1.335$ & $18.42 \pm 2.05$ & $-19.5 \pm 3.1$ & $\mathrm{~g}$ \\
\hline 101 & $16: 26: 30.8$ & $-24: 23: 47.10$ & $143.749 \pm 1.981$ & $14.878 \pm 1.313$ & $-9.170 \pm 1.314$ & $12.12 \pm 0.93$ & $-15.8 \pm 2.2$ & $\mathrm{~g}$ \\
\hline 102 & $16: 26: 25.5$ & $-24: 23: 47.10$ & $237.806 \pm 2.070$ & $6.513 \pm 1.263$ & $1.656 \pm 1.276$ & $2.78 \pm 0.53$ & $7.1 \pm 5.4$ & $\mathrm{f}$ \\
\hline 103 & $16: 26: 29.9$ & $-24: 23: 47.10$ & $304.683 \pm 2.024$ & $20.928 \pm 1.292$ & $-8.904 \pm 1.301$ & $7.45 \pm 0.43$ & $-11.5 \pm 1.6$ & $\mathrm{~g}$ \\
\hline 104 & $16: 26: 29.0$ & $-24: 23: 47.10$ & $761.543 \pm 2.091$ & $18.376 \pm 1.273$ & $-16.700 \pm 1.291$ & $3.26 \pm 0.17$ & $-21.1 \pm 1.5$ & a \\
\hline 105 & $16: 26: 26.4$ & $-24: 23: 47.10$ & $1388.390 \pm 2.214$ & $18.759 \pm 1.263$ & $-12.531 \pm 1.278$ & $1.62 \pm 0.09$ & $-16.9 \pm 1.6$ & $\mathrm{a}$ \\
\hline 106 & $16: 26: 28.1$ & $-24: 23: 47.10$ & $2694.310 \pm 2.563$ & $4.042 \pm 1.289$ & $-39.784 \pm 1.301$ & $1.48 \pm 0.05$ & $-42.1 \pm 0.9$ & $\mathrm{a}$ \\
\hline 107 & $16: 26: 27.3$ & $-24: 23: 47.10$ & $3342.890 \pm 2.437$ & $3.979 \pm 1.275$ & $-65.795 \pm 1.288$ & $1.97 \pm 0.04$ & $-43.3 \pm 0.6$ & $\mathrm{a}$ \\
\hline 108 & $16: 26: 21.1$ & $-24: 23: 47.09$ & $75.114 \pm 2.166$ & $0.361 \pm 1.367$ & $-3.412 \pm 1.369$ & $4.19 \pm 1.83$ & $-42.0 \pm 11.4$ & $f$ \\
\hline 109 & $16: 26: 22.0$ & $-24: 23: 47.09$ & $90.150 \pm 2.150$ & $-4.675 \pm 1.347$ & $-0.223 \pm 1.340$ & $4.97 \pm 1.50$ & $-88.6 \pm 8.2$ & $f$ \\
\hline 110 & $16: 26: 22.9$ & $-24: 23: 47.09$ & $104.711 \pm 2.086$ & $-8.144 \pm 1.315$ & $-2.148 \pm 1.329$ & $7.94 \pm 1.27$ & $-82.6 \pm 4.5$ & $f$ \\
\hline 111 & $16: 26: 17.6$ & $-24: 23: 47.07$ & $81.837 \pm 2.416$ & $-0.953 \pm 1.480$ & $4.201 \pm 1.473$ & $4.95 \pm 1.81$ & $51.4 \pm 9.8$ & $\ldots$ \\
\hline 112 & $16: 26: 24.6$ & $-24: 23: 35.10$ & $34.165 \pm 2.103$ & $5.500 \pm 1.287$ & $-0.679 \pm 1.299$ & $15.78 \pm 3.90$ & $-3.5 \pm 6.7$ & $f$ \\
\hline 113 & $16: 26: 30.8$ & $-24: 23: 35.10$ & $98.238 \pm 2.043$ & $14.844 \pm 1.313$ & $-4.484 \pm 1.322$ & $15.73 \pm 1.38$ & $-8.4 \pm 2.4$ & $\mathrm{~g}$ \\
\hline 114 & $16: 26: 29.9$ & $-24: 23: 35.10$ & $283.551 \pm 2.024$ & $18.141 \pm 1.301$ & $-3.264 \pm 1.311$ & $6.48 \pm 0.46$ & $-5.1 \pm 2.0$ & $\mathrm{~g}$ \\
\hline 115 & $16: 26: 25.5$ & $-24: 23: 35.10$ & $303.698 \pm 2.099$ & $3.975 \pm 1.272$ & $3.232 \pm 1.276$ & $1.63 \pm 0.42$ & $19.6 \pm 7.1$ & f \\
\hline 116 & $16: 26: 29.0$ & $-24: 23: 35.10$ & $676.103 \pm 2.073$ & $19.248 \pm 1.289$ & $-5.387 \pm 1.297$ & $2.95 \pm 0.19$ & $-7.8 \pm 1.9$ & $\mathrm{a}$ \\
\hline 117 & $16: 26: 26.4$ & $-24: 23: 35.10$ & $1357.650 \pm 2.208$ & $3.294 \pm 1.273$ & $-0.445 \pm 1.283$ & $0.23 \pm 0.09$ & $-3.8 \pm 11.1$ & $\mathrm{a}$ \\
\hline 118 & $16: 26: 28.1$ & $-24: 23: 35.10$ & $2104.370 \pm 2.360$ & $13.236 \pm 1.290$ & $-4.725 \pm 1.297$ & $0.67 \pm 0.06$ & $-9.8 \pm 2.6$ & $\mathrm{a}$ \\
\hline 119 & $16: 26: 27.3$ & $-24: 23: 35.10$ & $2889.410 \pm 2.241$ & $0.268 \pm 1.270$ & $-14.623 \pm 1.284$ & $0.50 \pm 0.04$ & $-44.5 \pm 2.5$ & $\mathrm{a}$ \\
\hline 120 & $16: 26: 22.9$ & $-24: 23: 35.09$ & $133.382 \pm 2.143$ & $-3.114 \pm 1.325$ & $-0.235 \pm 1.336$ & $2.12 \pm 0.99$ & $-87.8 \pm 12.3$ & $\mathrm{f}$ \\
\hline 121 & $16: 26: 21.1$ & $-24: 23: 35.09$ & $141.695 \pm 2.191$ & $-1.824 \pm 1.382$ & $-7.951 \pm 1.390$ & $5.67 \pm 0.98$ & $-51.5 \pm 4.9$ & $\mathrm{f}$ \\
\hline 122 & $16: 26: 22.0$ & $-24: 23: 35.09$ & $176.072 \pm 2.160$ & $-5.984 \pm 1.356$ & $-5.542 \pm 1.369$ & $4.57 \pm 0.78$ & $-68.6 \pm 4.8$ & $\mathrm{f}$ \\
\hline 123 & $16: 26: 20.2$ & $-24: 23: 35.08$ & $68.531 \pm 2.214$ & $-3.665 \pm 1.414$ & $-5.246 \pm 1.406$ & $9.11 \pm 2.08$ & $-62.5 \pm 6.3$ & $f$ \\
\hline 124 & $16: 26: 17.6$ & $-24: 23: 35.07$ & $45.658 \pm 2.459$ & $-3.891 \pm 1.486$ & $3.613 \pm 1.498$ & $11.16 \pm 3.33$ & $68.6 \pm 8.1$ & $\cdots$ \\
\hline 125 & $16: 26: 24.6$ & $-24: 23: 23.10$ & $72.227 \pm 2.084$ & $6.803 \pm 1.299$ & $-1.181 \pm 1.310$ & $9.39 \pm 1.82$ & $-4.9 \pm 5.4$ & e \\
\hline 126 & $16: 26: 30.8$ & $-24: 23: 23.10$ & $85.309 \pm 2.073$ & $10.189 \pm 1.334$ & $1.491 \pm 1.335$ & $11.97 \pm 1.59$ & $4.2 \pm 3.7$ & $\mathrm{~g}$ \\
\hline
\end{tabular}


Table 1

(Continued)

\begin{tabular}{|c|c|c|c|c|c|c|c|c|}
\hline \multirow[t]{2}{*}{ ID } & \multicolumn{2}{|c|}{ Position } & \multirow{2}{*}{$\begin{array}{c}I \pm \delta I \\
\left(\mathrm{mJy}^{-1} \text { beam }^{-1}\right)\end{array}$} & \multirow{2}{*}{$\begin{array}{c}Q \pm \delta Q \\
\left(\text { mJy beam }^{-1}\right)\end{array}$} & \multirow{2}{*}{$\begin{array}{c}U \pm \delta U \\
\left(\mathrm{mJy}^{-1} \text { beam }^{-1}\right)\end{array}$} & \multirow{2}{*}{$\begin{array}{c}P \pm \delta P \\
(\%)\end{array}$} & \multirow{2}{*}{$\begin{array}{c}\theta \pm \delta \theta \\
(\operatorname{deg})\end{array}$} & \multirow[t]{2}{*}{ Component } \\
\hline & $\alpha_{\mathrm{J} 2000}$ & $\delta_{\mathrm{J} 2000}$ & & & & & & \\
\hline 127 & $16: 26: 29.9$ & $-24: 23: 23.10$ & $310.310 \pm 2.079$ & $16.010 \pm 1.312$ & $-2.907 \pm 1.329$ & $5.23 \pm 0.42$ & $-5.1 \pm 2.3$ & $\mathrm{~g}$ \\
\hline 128 & $16: 26: 25.5$ & $-24: 23: 23.10$ & $321.875 \pm 2.090$ & $4.902 \pm 1.290$ & $-0.923 \pm 1.295$ & $1.50 \pm 0.40$ & $-5.3 \pm 7.4$ & $\mathrm{e}$ \\
\hline 129 & $16: 26: 29.0$ & $-24: 23: 23.10$ & $701.789 \pm 2.107$ & $18.357 \pm 1.302$ & $-0.381 \pm 1.323$ & $2.61 \pm 0.19$ & $-0.6 \pm 2.1$ & $\mathrm{e}$ \\
\hline 130 & $16: 26: 26.4$ & $-24: 23: 23.10$ & $1168.270 \pm 2.178$ & $2.500 \pm 1.283$ & $3.359 \pm 1.291$ & $0.34 \pm 0.11$ & $26.7 \pm 8.8$ & e \\
\hline 131 & $16: 26: 28.1$ & $-24: 23: 23.10$ & $1718.330 \pm 2.270$ & $19.074 \pm 1.298$ & $8.152 \pm 1.310$ & $1.20 \pm 0.08$ & $11.6 \pm 1.8$ & $\mathrm{e}$ \\
\hline 132 & $16: 26: 27.3$ & $-24: 23: 23.10$ & $2362.890 \pm 2.281$ & $10.365 \pm 1.290$ & $12.325 \pm 1.292$ & $0.68 \pm 0.05$ & $25.0 \pm 2.3$ & $\mathrm{e}$ \\
\hline 133 & $16: 26: 22.9$ & $-24: 23: 23.09$ & $97.956 \pm 2.203$ & $4.341 \pm 1.339$ & $-2.653 \pm 1.346$ & $5.01 \pm 1.37$ & $-15.7 \pm 7.6$ & $\mathrm{f}$ \\
\hline 134 & $16: 26: 21.1$ & $-24: 23: 23.09$ & $122.827 \pm 2.216$ & $1.495 \pm 1.410$ & $-7.600 \pm 1.403$ & $6.20 \pm 1.15$ & $-39.4 \pm 5.2$ & $\mathrm{f}$ \\
\hline 135 & $16: 26: 22.0$ & $-24: 23: 23.09$ & $162.363 \pm 2.222$ & $-0.593 \pm 1.368$ & $-4.353 \pm 1.383$ & $2.57 \pm 0.85$ & $-48.9 \pm 8.9$ & $\mathrm{f}$ \\
\hline 136 & $16: 26: 19.3$ & $-24: 23: 23.08$ & $40.275 \pm 2.341$ & $-6.088 \pm 1.449$ & $-3.108 \pm 1.464$ & $16.59 \pm 3.74$ & $-76.5 \pm 6.1$ & $\cdots$ \\
\hline 137 & $16: 26: 20.2$ & $-24: 23: 23.08$ & $66.519 \pm 2.301$ & $-1.848 \pm 1.412$ & $-5.787 \pm 1.421$ & $8.88 \pm 2.16$ & $-53.9 \pm 6.7$ & $\mathrm{f}$ \\
\hline 138 & $16: 26: 24.6$ & $-24: 23: 11.10$ & $87.122 \pm 2.106$ & $9.494 \pm 1.307$ & $-1.679 \pm 1.312$ & $10.96 \pm 1.52$ & $-5.0 \pm 3.9$ & $\mathrm{e}$ \\
\hline 139 & $16: 26: 30.8$ & $-24: 23: 11.10$ & $99.378 \pm 2.120$ & $7.089 \pm 1.338$ & $-2.624 \pm 1.359$ & $7.49 \pm 1.36$ & $-10.2 \pm 5.1$ & $\mathrm{~g}$ \\
\hline 140 & $16: 26: 25.5$ & $-24: 23: 11.10$ & $320.829 \pm 2.114$ & $12.169 \pm 1.306$ & $1.902 \pm 1.318$ & $3.82 \pm 0.41$ & $4.4 \pm 3.1$ & $\mathrm{e}$ \\
\hline 141 & $16: 26: 29.9$ & $-24: 23: 11.10$ & $335.974 \pm 2.101$ & $14.871 \pm 1.338$ & $-7.919 \pm 1.346$ & $5.00 \pm 0.40$ & $-14.0 \pm 2.3$ & $\mathrm{~g}$ \\
\hline 142 & $16: 26: 29.0$ & $-24: 23: 11.10$ & $686.497 \pm 2.134$ & $17.110 \pm 1.316$ & $6.580 \pm 1.322$ & $2.66 \pm 0.19$ & $10.5 \pm 2.1$ & e \\
\hline 143 & $16: 26: 26.4$ & $-24: 23: 11.10$ & $815.059 \pm 2.143$ & $14.348 \pm 1.306$ & $7.082 \pm 1.316$ & $1.96 \pm 0.16$ & $13.1 \pm 2.4$ & $\mathrm{e}$ \\
\hline 144 & $16: 26: 28.1$ & $-24: 23: 11.10$ & $1001.860 \pm 2.132$ & $20.516 \pm 1.311$ & $14.985 \pm 1.322$ & $2.53 \pm 0.13$ & $18.1 \pm 1.5$ & $\mathrm{e}$ \\
\hline 145 & $16: 26: 27.3$ & $-24: 23: 11.10$ & $1208.520 \pm 2.179$ & $16.976 \pm 1.303$ & $20.285 \pm 1.320$ & $2.19 \pm 0.11$ & $25.0 \pm 1.4$ & $\mathrm{e}$ \\
\hline 146 & $16: 26: 23.7$ & $-24: 23: 11.09$ & $36.854 \pm 2.139$ & $7.226 \pm 1.329$ & $-0.484 \pm 1.343$ & $19.32 \pm 3.78$ & $-1.9 \pm 5.3$ & $\mathrm{e}$ \\
\hline 147 & $16: 26: 22.9$ & $-24: 23: 11.09$ & $101.073 \pm 2.259$ & $8.288 \pm 1.362$ & $-2.030 \pm 1.374$ & $8.33 \pm 1.36$ & $-6.9 \pm 4.6$ & $\mathrm{f}$ \\
\hline 148 & $16: 26: 21.1$ & $-24: 23: 11.09$ & $110.047 \pm 2.299$ & $6.195 \pm 1.405$ & $-5.566 \pm 1.409$ & $7.46 \pm 1.29$ & $-21.0 \pm 4.8$ & $\mathrm{f}$ \\
\hline 149 & $16: 26: 22.0$ & $-24: 23: 11.09$ & $193.122 \pm 2.272$ & $4.598 \pm 1.386$ & $-1.217 \pm 1.401$ & $2.36 \pm 0.72$ & $-7.4 \pm 8.4$ & $\mathrm{f}$ \\
\hline 150 & $16: 26: 20.2$ & $-24: 23: 11.08$ & $41.152 \pm 2.353$ & $2.214 \pm 1.423$ & $-5.069 \pm 1.439$ & $12.98 \pm 3.57$ & $-33.2 \pm 7.4$ & $\mathrm{f}$ \\
\hline 151 & $16: 26: 31.6$ & $-24: 22: 59.10$ & $37.752 \pm 2.203$ & $3.501 \pm 1.386$ & $4.052 \pm 1.401$ & $13.70 \pm 3.79$ & $24.6 \pm 7.4$ & $\mathrm{~g}$ \\
\hline 152 & $16: 26: 24.6$ & $-24: 22: 59.10$ & $71.414 \pm 2.169$ & $5.972 \pm 1.330$ & $2.543 \pm 1.344$ & $8.89 \pm 1.89$ & $11.5 \pm 5.9$ & $\mathrm{e}$ \\
\hline 153 & $16: 26: 30.8$ & $-24: 22: 59.10$ & $138.114 \pm 2.176$ & $0.977 \pm 1.363$ & $-5.578 \pm 1.384$ & $3.98 \pm 1.00$ & $-40.0 \pm 6.9$ & $\mathrm{~g}$ \\
\hline 154 & $16: 26: 25.5$ & $-24: 22: 59.10$ & $304.060 \pm 2.147$ & $13.581 \pm 1.329$ & $3.681 \pm 1.338$ & $4.61 \pm 0.44$ & $7.6 \pm 2.7$ & $\mathrm{e}$ \\
\hline 155 & $16: 26: 29.9$ & $-24: 22: 59.10$ & $369.449 \pm 2.144$ & $7.799 \pm 1.361$ & $-11.722 \pm 1.355$ & $3.79 \pm 0.37$ & $-28.2 \pm 2.8$ & g \\
\hline 156 & $16: 26: 26.4$ & $-24: 22: 59.10$ & $532.961 \pm 2.154$ & $20.744 \pm 1.328$ & $14.188 \pm 1.338$ & $4.71 \pm 0.25$ & $17.2 \pm 1.5$ & $\mathrm{e}$ \\
\hline 157 & $16: 26: 29.0$ & $-24: 22: 59.10$ & $720.738 \pm 2.125$ & $16.729 \pm 1.329$ & $4.677 \pm 1.349$ & $2.40 \pm 0.18$ & $7.8 \pm 2.2$ & $\mathrm{e}$ \\
\hline 158 & $16: 26: 27.3$ & $-24: 22: 59.10$ & $744.220 \pm 2.160$ & $25.034 \pm 1.322$ & $24.150 \pm 1.348$ & $4.67 \pm 0.18$ & $22.0 \pm 1.1$ & $\mathrm{e}$ \\
\hline 159 & $16: 26: 28.1$ & $-24: 22: 59.10$ & $904.956 \pm 2.147$ & $26.453 \pm 1.329$ & $14.877 \pm 1.338$ & $3.35 \pm 0.15$ & $14.7 \pm 1.3$ & e \\
\hline 160 & $16: 26: 22.9$ & $-24: 22: 59.09$ & $91.979 \pm 2.279$ & $9.092 \pm 1.376$ & $-3.091 \pm 1.397$ & $10.33 \pm 1.52$ & $-9.4 \pm 4.2$ & $\mathrm{f}$ \\
\hline 161 & $16: 26: 21.1$ & $-24: 22: 59.09$ & $222.216 \pm 2.338$ & $13.295 \pm 1.406$ & $-6.026 \pm 1.429$ & $6.54 \pm 0.64$ & $-12.2 \pm 2.8$ & $\mathrm{f}$ \\
\hline 162 & $16: 26: 22.0$ & $-24: 22: 59.09$ & $345.663 \pm 2.325$ & $15.068 \pm 1.400$ & $-3.957 \pm 1.413$ & $4.49 \pm 0.41$ & $-7.4 \pm 2.6$ & $\mathrm{f}$ \\
\hline 163 & $16: 26: 20.2$ & $-24: 22: 59.08$ & $60.825 \pm 2.358$ & $9.465 \pm 1.441$ & $-2.125 \pm 1.467$ & $15.77 \pm 2.45$ & $-6.3 \pm 4.3$ & $\mathrm{f}$ \\
\hline 164 & $16: 26: 45.7$ & $-24: 22: 59.05$ & $88.311 \pm 3.019$ & $4.615 \pm 2.132$ & $2.294 \pm 2.148$ & $5.31 \pm 2.43$ & $13.2 \pm 11.9$ & $\ldots$ \\
\hline 165 & $16: 26: 11.4$ & $-24: 22: 59.04$ & $48.636 \pm 3.084$ & $-0.765 \pm 1.829$ & $-5.855 \pm 1.843$ & $11.53 \pm 3.87$ & $-48.7 \pm 8.9$ & $\ldots$ \\
\hline 166 & $16: 26: 24.6$ & $-24: 22: 47.10$ & $53.074 \pm 2.265$ & $9.215 \pm 1.363$ & $1.841 \pm 1.366$ & $17.52 \pm 2.68$ & $5.6 \pm 4.2$ & $\mathrm{e}$ \\
\hline 167 & $16: 26: 30.8$ & $-24: 22: 47.10$ & $134.127 \pm 2.222$ & $3.846 \pm 1.393$ & $-9.789 \pm 1.399$ & $7.77 \pm 1.05$ & $-34.3 \pm 3.8$ & $\mathrm{~b}$ \\
\hline 168 & $16: 26: 25.5$ & $-24: 22: 47.10$ & $180.227 \pm 2.233$ & $15.879 \pm 1.353$ & $10.538 \pm 1.373$ & $10.55 \pm 0.77$ & $16.8 \pm 2.1$ & $\mathrm{e}$ \\
\hline 169 & $16: 26: 26.4$ & $-24: 22: 47.10$ & $381.385 \pm 2.224$ & $19.126 \pm 1.354$ & $14.805 \pm 1.361$ & $6.33 \pm 0.36$ & $18.9 \pm 1.6$ & $\mathrm{e}$ \\
\hline 170 & $16: 26: 29.9$ & $-24: 22: 47.10$ & $407.585 \pm 2.203$ & $6.420 \pm 1.378$ & $-11.779 \pm 1.384$ & $3.27 \pm 0.34$ & $-30.7 \pm 2.9$ & $\mathrm{~b}$ \\
\hline 171 & $16: 26: 27.3$ & $-24: 22: 47.10$ & $585.774 \pm 2.234$ & $13.418 \pm 1.348$ & $13.767 \pm 1.370$ & $3.27 \pm 0.23$ & $22.9 \pm 2.0$ & $\mathrm{e}$ \\
\hline 172 & $16: 26: 29.0$ & $-24: 22: 47.10$ & $658.788 \pm 2.224$ & $7.521 \pm 1.370$ & $-4.418 \pm 1.374$ & $1.31 \pm 0.21$ & $-15.2 \pm 4.5$ & $\mathrm{e}$ \\
\hline 173 & $16: 26: 28.1$ & $-24: 22: 47.10$ & $739.262 \pm 2.188$ & $9.876 \pm 1.351$ & $8.635 \pm 1.360$ & $1.77 \pm 0.18$ & $20.6 \pm 3.0$ & $\mathrm{e}$ \\
\hline 174 & $16: 26: 23.7$ & $-24: 22: 47.09$ & $38.876 \pm 2.281$ & $8.239 \pm 1.371$ & $-3.363 \pm 1.383$ & $22.62 \pm 3.78$ & $-11.1 \pm 4.4$ & $\mathrm{e}$ \\
\hline 175 & $16: 26: 22.9$ & $-24: 22: 47.09$ & $159.157 \pm 2.341$ & $9.114 \pm 1.389$ & $-4.033 \pm 1.406$ & $6.20 \pm 0.88$ & $-11.9 \pm 4.0$ & $\mathrm{f}$ \\
\hline 176 & $16: 26: 21.1$ & $-24: 22: 47.09$ & $314.588 \pm 2.401$ & $12.322 \pm 1.422$ & $-3.301 \pm 1.436$ & $4.03 \pm 0.45$ & $-7.5 \pm 3.2$ & $\mathrm{f}$ \\
\hline 177 & $16: 26: 22.0$ & $-24: 22: 47.09$ & $413.865 \pm 2.349$ & $8.794 \pm 1.403$ & $-10.808 \pm 1.419$ & $3.35 \pm 0.34$ & $-25.4 \pm 2.9$ & $\mathrm{f}$ \\
\hline 178 & $16: 26: 19.3$ & $-24: 22: 47.08$ & $59.614 \pm 2.493$ & $7.615 \pm 1.482$ & $-4.514 \pm 1.498$ & $14.64 \pm 2.57$ & $-15.3 \pm 4.8$ & $\ldots$ \\
\hline 179 & $16: 26: 20.2$ & $-24: 22: 47.08$ & $156.319 \pm 2.393$ & $9.025 \pm 1.453$ & $-2.469 \pm 1.475$ & $5.91 \pm 0.94$ & $-7.7 \pm 4.5$ & $\mathrm{f}$ \\
\hline 180 & $16: 26: 24.6$ & $-24: 22: 35.10$ & $127.395 \pm 2.270$ & $14.736 \pm 1.376$ & $2.503 \pm 1.397$ & $11.68 \pm 1.10$ & $4.8 \pm 2.7$ & $\mathrm{e}$ \\
\hline 181 & $16: 26: 30.8$ & $-24: 22: 35.10$ & $212.186 \pm 2.256$ & $3.442 \pm 1.415$ & $-19.852 \pm 1.426$ & $9.47 \pm 0.68$ & $-40.1 \pm 2.0$ & $\mathrm{~b}$ \\
\hline 182 & $16: 26: 25.5$ & $-24: 22: 35.10$ & $251.386 \pm 2.291$ & $16.480 \pm 1.379$ & $8.631 \pm 1.404$ & $7.38 \pm 0.55$ & $13.8 \pm 2.2$ & $\mathrm{e}$ \\
\hline 183 & $16: 26: 26.4$ & $-24: 22: 35.10$ & $413.479 \pm 2.280$ & $11.576 \pm 1.375$ & $5.455 \pm 1.392$ & $3.08 \pm 0.33$ & $12.6 \pm 3.1$ & e \\
\hline 184 & $16: 26: 29.9$ & $-24: 22: 35.10$ & $456.761 \pm 2.252$ & $5.149 \pm 1.392$ & $-9.420 \pm 1.406$ & $2.33 \pm 0.31$ & $-30.7 \pm 3.7$ & $\mathrm{~b}$ \\
\hline 185 & $16: 26: 27.3$ & $-24: 22: 35.10$ & $455.718 \pm 2.279$ & $4.230 \pm 1.381$ & $1.790 \pm 1.391$ & $0.96 \pm 0.30$ & $11.5 \pm 8.7$ & $\mathrm{e}$ \\
\hline 186 & $16: 26: 23.7$ & $-24: 22: 35.09$ & $104.526 \pm 2.323$ & $11.715 \pm 1.390$ & $-3.032 \pm 1.401$ & $11.50 \pm 1.35$ & $-7.3 \pm 3.3$ & e \\
\hline 187 & $16: 26: 22.9$ & $-24: 22: 35.09$ & $178.125 \pm 2.360$ & $6.971 \pm 1.401$ & $-1.650 \pm 1.422$ & $3.94 \pm 0.79$ & $-6.7 \pm 5.7$ & $\mathrm{f}$ \\
\hline 188 & $16: 26: 21.1$ & $-24: 22: 35.09$ & $220.112 \pm 2.435$ & $8.737 \pm 1.443$ & $-1.179 \pm 1.461$ & $3.95 \pm 0.66$ & $-3.8 \pm 4.7$ & $\mathrm{f}$ \\
\hline 189 & $16: 26: 22.0$ & $-24: 22: 35.09$ & $261.877 \pm 2.396$ & $8.756 \pm 1.426$ & $-5.262 \pm 1.445$ & $3.86 \pm 0.55$ & $-15.5 \pm 4.0$ & $\mathrm{f}$ \\
\hline
\end{tabular}


Table 1

(Continued)

\begin{tabular}{|c|c|c|c|c|c|c|c|c|}
\hline \multirow[t]{2}{*}{ ID } & \multicolumn{2}{|c|}{ Position } & \multirow{2}{*}{$\begin{array}{c}I \pm \delta I \\
\left(\mathrm{mJy} \mathrm{beam}^{-1}\right)\end{array}$} & \multirow{2}{*}{$\begin{array}{c}Q \pm \delta Q \\
\left(\mathrm{mJy}^{-1} \text { beam }^{-1}\right)\end{array}$} & \multirow{2}{*}{$\begin{array}{c}U \pm \delta U \\
\left(\mathrm{mJy}^{-1} \text { beam }^{-1}\right)\end{array}$} & \multirow{2}{*}{$\begin{array}{l}P \pm \delta P \\
(\%)\end{array}$} & \multirow{2}{*}{$\begin{array}{c}\theta \pm \delta \theta \\
(\operatorname{deg})\end{array}$} & \multirow{2}{*}{ Component } \\
\hline & $\alpha_{\mathrm{J} 2000}$ & $\delta_{\mathbf{J} 2000}$ & & & & & & \\
\hline 190 & $16: 26: 19.3$ & $-24: 22: 35.08$ & $56.937 \pm 2.484$ & $4.730 \pm 1.488$ & $-2.345 \pm 1.503$ & $8.90 \pm 2.65$ & $-13.2 \pm 8.1$ & $\cdots$ \\
\hline 191 & $16: 26: 20.2$ & $-24: 22: 35.08$ & $125.177 \pm 2.428$ & $9.520 \pm 1.454$ & $-1.563 \pm 1.467$ & $7.62 \pm 1.17$ & $-4.7 \pm 4.4$ & $\mathrm{f}$ \\
\hline 192 & $16: 26: 31.6$ & $-24: 22: 23.10$ & $87.998 \pm 2.335$ & $-0.555 \pm 1.462$ & $-14.397 \pm 1.483$ & $16.29 \pm 1.74$ & $-46.1 \pm 2.9$ & $\mathrm{~b}$ \\
\hline 193 & $16: 26: 24.6$ & $-24: 22: 23.10$ & $235.612 \pm 2.314$ & $8.555 \pm 1.405$ & $5.685 \pm 1.427$ & $4.32 \pm 0.60$ & $16.8 \pm 4.0$ & $\mathrm{~d}$ \\
\hline 194 & $16: 26: 30.8$ & $-24: 22: 23.10$ & $265.974 \pm 2.288$ & $1.192 \pm 1.444$ & $-14.088 \pm 1.458$ & $5.29 \pm 0.55$ & $-42.6 \pm 2.9$ & $\mathrm{~b}$ \\
\hline 195 & $16: 26: 28.1$ & $-24: 22: 23.10$ & $321.044 \pm 2.315$ & $-9.868 \pm 1.410$ & $-4.661 \pm 1.431$ & $3.37 \pm 0.44$ & $-77.4 \pm 3.7$ & $\mathrm{c}$ \\
\hline 196 & $16: 26: 25.5$ & $-24: 22: 23.10$ & $327.336 \pm 2.300$ & $7.121 \pm 1.404$ & $3.980 \pm 1.421$ & $2.45 \pm 0.43$ & $14.6 \pm 5.0$ & $\mathrm{~d}$ \\
\hline 197 & $16: 26: 29.0$ & $-24: 22: 23.10$ & $356.454 \pm 2.331$ & $-5.714 \pm 1.432$ & $-1.487 \pm 1.440$ & $1.61 \pm 0.40$ & $-82.7 \pm 7.0$ & $\mathrm{c}$ \\
\hline 198 & $16: 26: 29.9$ & $-24: 22: 23.10$ & $376.302 \pm 2.288$ & $-4.820 \pm 1.422$ & $-5.187 \pm 1.446$ & $1.84 \pm 0.38$ & $-66.4 \pm 5.8$ & $\mathrm{~b}$ \\
\hline 199 & $16: 26: 27.3$ & $-24: 22: 23.10$ & $393.647 \pm 2.295$ & $-3.922 \pm 1.410$ & $-4.097 \pm 1.413$ & $1.40 \pm 0.36$ & $-66.9 \pm 7.1$ & $\mathrm{c}$ \\
\hline 200 & $16: 26: 21.1$ & $-24: 22: 23.09$ & $121.082 \pm 2.429$ & $7.619 \pm 1.462$ & $3.376 \pm 1.476$ & $6.78 \pm 1.22$ & $11.9 \pm 5.1$ & $\mathrm{~d}$ \\
\hline 201 & $16: 26: 23.7$ & $-24: 22: 23.09$ & $163.410 \pm 2.324$ & $8.735 \pm 1.416$ & $3.330 \pm 1.424$ & $5.65 \pm 0.87$ & $10.4 \pm 4.4$ & $\mathrm{~d}$ \\
\hline 202 & $16: 26: 22.0$ & $-24: 22: 23.09$ & $181.200 \pm 2.453$ & $3.215 \pm 1.440$ & $2.708 \pm 1.459$ & $2.18 \pm 0.80$ & $20.1 \pm 9.9$ & $\mathrm{~d}$ \\
\hline 203 & $16: 26: 22.9$ & $-24: 22: 23.09$ & $182.764 \pm 2.444$ & $5.491 \pm 1.429$ & $6.321 \pm 1.438$ & $4.51 \pm 0.79$ & $24.5 \pm 4.9$ & $\mathrm{~d}$ \\
\hline 204 & $16: 26: 20.2$ & $-24: 22: 23.08$ & $52.899 \pm 2.470$ & $3.222 \pm 1.474$ & $2.652 \pm 1.488$ & $7.38 \pm 2.82$ & $19.7 \pm 10.2$ & $\mathrm{~d}$ \\
\hline 205 & $16: 26: 32.5$ & $-24: 22: 11.10$ & $48.267 \pm 2.362$ & $-1.336 \pm 1.509$ & $-9.367 \pm 1.509$ & $19.35 \pm 3.27$ & $-49.1 \pm 4.6$ & $\mathrm{~b}$ \\
\hline 206 & $16: 26: 29.0$ & $-24: 22: 11.10$ & $143.786 \pm 2.370$ & $-10.132 \pm 1.461$ & $-0.830 \pm 1.466$ & $7.00 \pm 1.02$ & $-87.7 \pm 4.1$ & $\mathrm{c}$ \\
\hline 207 & $16: 26: 31.6$ & $-24: 22: 11.10$ & $154.485 \pm 2.387$ & $-2.150 \pm 1.496$ & $-16.003 \pm 1.504$ & $10.41 \pm 0.99$ & $-48.8 \pm 2.7$ & $\mathrm{~b}$ \\
\hline 208 & $16: 26: 28.1$ & $-24: 22: 11.10$ & $160.939 \pm 2.374$ & $-9.426 \pm 1.444$ & $-2.592 \pm 1.456$ & $6.01 \pm 0.90$ & $-82.3 \pm 4.3$ & $\mathrm{c}$ \\
\hline 209 & $16: 26: 29.9$ & $-24: 22: 11.10$ & $222.641 \pm 2.385$ & $-3.662 \pm 1.455$ & $-6.375 \pm 1.471$ & $3.24 \pm 0.66$ & $-59.9 \pm 5.7$ & $\mathrm{~b}$ \\
\hline 210 & $16: 26: 24.6$ & $-24: 22: 11.10$ & $234.259 \pm 2.338$ & $8.571 \pm 1.436$ & $3.194 \pm 1.436$ & $3.86 \pm 0.61$ & $10.2 \pm 4.5$ & $\mathrm{~d}$ \\
\hline 211 & $16: 26: 30.8$ & $-24: 22: 11.10$ & $247.756 \pm 2.364$ & $-7.141 \pm 1.472$ & $-18.687 \pm 1.488$ & $8.05 \pm 0.60$ & $-55.5 \pm 2.1$ & $\mathrm{~b}$ \\
\hline 212 & $16: 26: 27.3$ & $-24: 22: 11.10$ & $253.879 \pm 2.372$ & $-10.082 \pm 1.438$ & $-5.984 \pm 1.451$ & $4.58 \pm 0.57$ & $-74.7 \pm 3.5$ & $\mathrm{c}$ \\
\hline 213 & $16: 26: 25.5$ & $-24: 22: 11.10$ & $268.096 \pm 2.378$ & $2.588 \pm 1.436$ & $2.764 \pm 1.451$ & $1.31 \pm 0.54$ & $23.4 \pm 10.9$ & $\mathrm{~d}$ \\
\hline 214 & $16: 26: 22.0$ & $-24: 22: 11.09$ & $97.438 \pm 2.497$ & $0.420 \pm 1.441$ & $4.959 \pm 1.464$ & $4.88 \pm 1.51$ & $42.6 \pm 8.3$ & $\mathrm{~d}$ \\
\hline 215 & $16: 26: 22.9$ & $-24: 22: 11.09$ & $154.229 \pm 2.455$ & $6.446 \pm 1.441$ & $9.270 \pm 1.460$ & $7.26 \pm 0.95$ & $27.6 \pm 3.7$ & $\mathrm{~d}$ \\
\hline 216 & $16: 26: 23.7$ & $-24: 22: 11.09$ & $186.026 \pm 2.417$ & $8.161 \pm 1.437$ & $4.268 \pm 1.452$ & $4.89 \pm 0.78$ & $13.8 \pm 4.5$ & $\mathrm{~d}$ \\
\hline 217 & $16: 26: 28.1$ & $-24: 21: 59.10$ & $44.226 \pm 2.375$ & $-7.979 \pm 1.483$ & $-0.338 \pm 1.496$ & $17.74 \pm 3.49$ & $-88.8 \pm 5.4$ & $\mathrm{c}$ \\
\hline 218 & $16: 26: 32.5$ & $-24: 21: 59.10$ & $62.207 \pm 2.364$ & $1.265 \pm 1.538$ & $-7.122 \pm 1.549$ & $11.36 \pm 2.53$ & $-40.0 \pm 6.1$ & $\mathrm{~b}$ \\
\hline 219 & $16: 26: 29.9$ & $-24: 21: 59.10$ & $92.685 \pm 2.414$ & $-4.357 \pm 1.499$ & $3.663 \pm 1.503$ & $5.92 \pm 1.63$ & $70.0 \pm 7.6$ & $\mathrm{~b}$ \\
\hline 220 & $16: 26: 27.3$ & $-24: 21: 59.10$ & $147.180 \pm 2.391$ & $-7.698 \pm 1.476$ & $-8.673 \pm 1.491$ & $7.81 \pm 1.02$ & $-65.8 \pm 3.7$ & $\mathrm{c}$ \\
\hline 221 & $16: 26: 31.6$ & $-24: 21: 59.10$ & $161.920 \pm 2.428$ & $-1.451 \pm 1.507$ & $-11.238 \pm 1.529$ & $6.93 \pm 0.95$ & $-48.7 \pm 3.8$ & $\mathrm{~b}$ \\
\hline 222 & $16: 26: 30.8$ & $-24: 21: 59.10$ & $190.935 \pm 2.390$ & $-2.690 \pm 1.498$ & $-11.650 \pm 1.517$ & $6.21 \pm 0.80$ & $-51.5 \pm 3.6$ & $\mathrm{~b}$ \\
\hline 223 & $16: 26: 26.4$ & $-24: 21: 59.10$ & $205.434 \pm 2.365$ & $-0.648 \pm 1.464$ & $-5.748 \pm 1.466$ & $2.72 \pm 0.71$ & $-48.2 \pm 7.3$ & $\mathrm{c}$ \\
\hline 224 & $16: 26: 24.6$ & $-24: 21: 59.10$ & $239.257 \pm 2.468$ & $6.322 \pm 1.458$ & $1.838 \pm 1.478$ & $2.68 \pm 0.61$ & $8.1 \pm 6.4$ & $\mathrm{~d}$ \\
\hline 225 & $16: 26: 22.9$ & $-24: 21: 59.09$ & $148.213 \pm 2.557$ & $8.257 \pm 1.467$ & $6.551 \pm 1.499$ & $7.04 \pm 1.01$ & $19.2 \pm 4.0$ & $\mathrm{~d}$ \\
\hline 226 & $16: 26: 23.7$ & $-24: 21: 59.09$ & $242.340 \pm 2.485$ & $10.178 \pm 1.458$ & $5.633 \pm 1.468$ & $4.76 \pm 0.60$ & $14.5 \pm 3.6$ & $\mathrm{~d}$ \\
\hline 227 & $16: 26: 32.5$ & $-24: 21: 47.10$ & $44.850 \pm 2.477$ & $-5.374 \pm 1.581$ & $-7.263 \pm 1.592$ & $19.83 \pm 3.71$ & $-63.2 \pm 5.0$ & $\mathrm{~b}$ \\
\hline 228 & $16: 26: 27.3$ & $-24: 21: 47.10$ & $49.048 \pm 2.464$ & $-3.068 \pm 1.502$ & $-4.613 \pm 1.514$ & $10.87 \pm 3.13$ & $-61.8 \pm 7.8$ & $\mathrm{c}$ \\
\hline 229 & $16: 26: 30.8$ & $-24: 21: 47.10$ & $75.361 \pm 2.418$ & $-3.993 \pm 1.540$ & $-5.164 \pm 1.555$ & $8.41 \pm 2.07$ & $-63.9 \pm 6.8$ & $\mathrm{~b}$ \\
\hline 230 & $16: 26: 31.6$ & $-24: 21: 47.10$ & $98.617 \pm 2.441$ & $-6.708 \pm 1.555$ & $-8.407 \pm 1.571$ & $10.79 \pm 1.61$ & $-64.3 \pm 4.2$ & $\mathrm{~b}$ \\
\hline 231 & $16: 26: 26.4$ & $-24: 21: 47.10$ & $141.314 \pm 2.503$ & $-2.150 \pm 1.490$ & $-4.842 \pm 1.518$ & $3.59 \pm 1.07$ & $-57.0 \pm 8.1$ & $\mathrm{c}$ \\
\hline 232 & $16: 26: 22.9$ & $-24: 21: 47.09$ & $81.893 \pm 2.610$ & $2.848 \pm 1.503$ & $4.478 \pm 1.528$ & $6.21 \pm 1.87$ & $28.8 \pm 8.2$ & $\mathrm{~d}$ \\
\hline 233 & $16: 26: 23.7$ & $-24: 21: 47.09$ & $196.629 \pm 2.582$ & $4.398 \pm 1.501$ & $2.858 \pm 1.505$ & $2.56 \pm 0.76$ & $16.5 \pm 8.2$ & $\mathrm{~d}$ \\
\hline 234 & $16: 26: 26.4$ & $-24: 21: 35.10$ & $84.813 \pm 2.582$ & $-3.771 \pm 1.539$ & $-4.027 \pm 1.558$ & $6.24 \pm 1.84$ & $-66.6 \pm 8.0$ & $\mathrm{c}$ \\
\hline 235 & $16: 26: 23.7$ & $-24: 21: 35.09$ & $90.810 \pm 2.636$ & $3.895 \pm 1.538$ & $3.342 \pm 1.551$ & $5.39 \pm 1.71$ & $20.3 \pm 8.6$ & $\mathrm{~d}$ \\
\hline 236 & $16: 26: 24.6$ & $-24: 21: 11.10$ & $47.959 \pm 2.837$ & $-4.625 \pm 1.611$ & $2.377 \pm 1.643$ & $10.31 \pm 3.43$ & $76.4 \pm 9.0$ & $\mathrm{~d}$ \\
\hline 237 & $16: 26: 10.6$ & $-24: 19: 35.03$ & $77.061 \pm 4.747$ & $-2.700 \pm 2.625$ & $5.578 \pm 2.565$ & $7.31 \pm 3.38$ & $57.9 \pm 12.1$ & $\ldots$ \\
\hline 238 & $16: 26: 43.9$ & $-24: 17: 35.06$ & $124.438 \pm 7.065$ & $13.784 \pm 4.443$ & $-2.667 \pm 4.528$ & $10.70 \pm 3.63$ & $-5.5 \pm 9.2$ & $\ldots$ \\
\hline 239 & $16: 26: 43.1$ & $-24: 17: 23.06$ & $134.654 \pm 7.249$ & $15.535 \pm 4.650$ & $-0.423 \pm 4.745$ & $11.01 \pm 3.51$ & $-0.8 \pm 8.7$ & $\ldots$ \\
\hline 240 & $16: 26: 43.9$ & $-24: 17: 23.06$ & $170.148 \pm 7.541$ & $21.291 \pm 4.948$ & $-2.326 \pm 5.059$ & $12.25 \pm 2.96$ & $-3.1 \pm 6.8$ & $\ldots$ \\
\hline
\end{tabular}

Note. Units of right ascension are hours, minutes, and seconds, and units of declination are degrees, arcminutes, and arcseconds. Positions are from the Stokes $I$ image (Figure 1), and these sources are sorted by declination. Sources with $I>0, P / \delta P>2$, and $\delta P<4$ are listed. "Component" refers to regions shown in Figure 8.

(This table is available in machine-readable form.)

selected with the polarization $\mathrm{S} / \mathrm{N}(P / \delta P>2$ or 3$)$, and the other selected with the intensity $\mathrm{S} / \mathrm{N}(I / \delta I>20)$. The latter intensity selection is shown because selecting by $\mathrm{S} / \mathrm{N}$ in $P$ will tend to bias the polarization data sample to high $P$ values (especially toward regions of low intensity), so a comparison with a sample selected by $I / \delta I$ is made to show that without this bias, the polarization fraction is still larger on average for cloud sightlines in the envelope. Figure 7 demonstrates that this correlation is robust, as also seen from the negative correlation between the degrees of polarization and intensities in both the $P$ and $I$ selection data. We find that $P \propto I^{\gamma}$, where $\gamma \sim-0.8$ for the $P$ selection and -0.7 for the $I$ selection. Note that there is a 


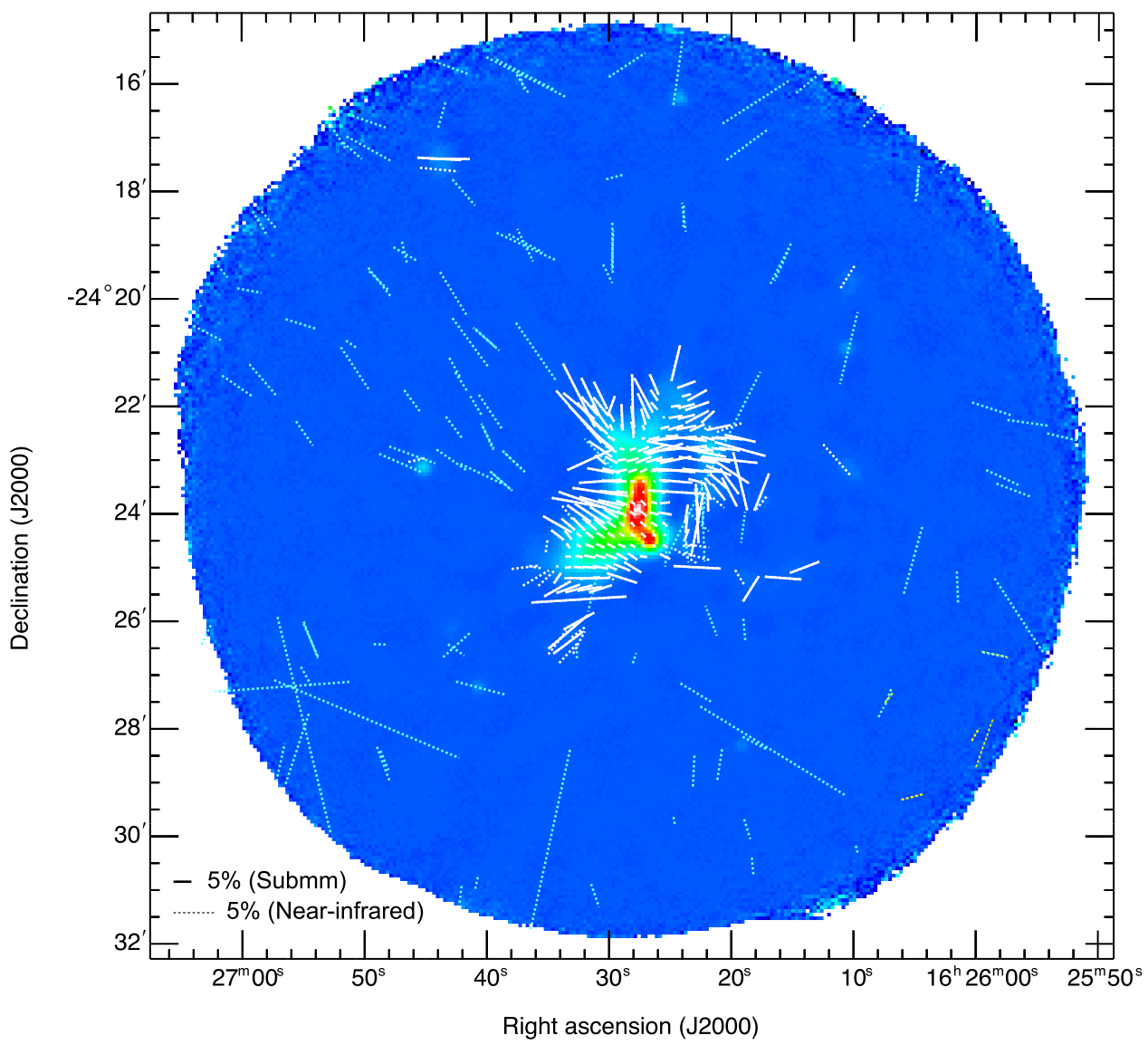

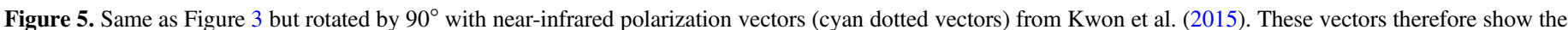

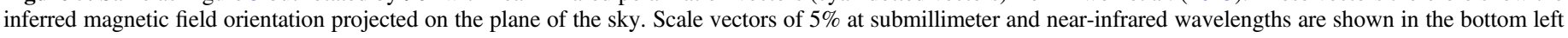
corner.

larger dispersion at the low-intensity regions in the $I$ selection because not only high $P$ data but also several low $P$ data exist in the $I$ selection. This trend might be due to a combination of several factors, such grain alignment and magnetic field geometry. A detailed discussion will be presented elsewhere.

We have found by eye that there are at least 10 distinct magnetic field components in the core region, and we refer to them as "components a-j" (see Figure 8). Please note that our division of these components does not mean that these field components are always independent, but all or some of them could be smoothly connected with each other. The aim of the region division here is mainly to identify the change of directions and degrees of the polarization vectors and to compare them with the near-infrared polarization data. A summary of these components is as follows.

(a) Small $P(<3 \%)$ and $\sim 50^{\circ}$ component at SM1 and VLA 1623 around the center of the observed field of view.

(b) Large $P$ and $\sim 40^{\circ}$ component near A-MM 7 to the east of A-MM 5.

(c) Large $P$ and $\sim 20^{\circ}$ component near A-MM 5 .

(d) Large $P$ and $\sim 100^{\circ}$ component at A-MM 4.

(e) Small $P(<3 \%)$ and $\sim 100^{\circ}$ component between A-MM 5 and SM1N.

(f) Large $P$ and $\sim 80^{\circ}$ component to the west of SM1.

(g) Large $P$ and $\sim 70^{\circ}$ component to the east of SM1 and SM1N.

(h) Large $P$ and $\sim 80^{\circ}$ component at A-MM 3 . (i) Small $P(<3 \%)$ and $\sim 80^{\circ}$ component between SM2 and A-MM 8.

(j) Large $P$ and $\sim 120^{\circ}$ component between SM2 and A-MM 8.

Figure 8 illustrates that these components differ from each other either in polarization position angle or degree of polarization (see also Table 2). Components a, c, and i are already seen in and consistent with the SCUPOL data (Tamura 1999). Components b, d, e, f, g, h, and j are additionally identified in our SCUBA-2/POL-2 data. One can also see the polarization vectors associated with components $\mathrm{b}, \mathrm{d}, \mathrm{e}, \mathrm{g}$, and $\mathrm{j}$ in Matthews et al. (2009). We also note that our results suggest that the magnetic field is mostly well organized (rather than disordered due to turbulence; see Section 5.2).

In the central region around SM1 (component a), the vectors are well aligned with the $50^{\circ}$ magnetic field component observed in the surrounding medium on various scales (see Section 5.4). Although the average direction of the main component is approximately $50^{\circ}$, the magnetic field tends to be locally perpendicular (approximately $100^{\circ}-110^{\circ}$ ) to the arcstructure (south part of region f). Between SM1N and A-MM 6, the magnetic field direction is almost east-west (component e), while the arc extends to the northeast or northwest, and the magnetic field directions extend toward LFAM 1 and GSS 30-1 (component f). A perpendicular field relative to the core shape (i.e., the elongation of the arcstructure between the northeast and northwest filaments) is important for the formation and growth of this core. Such orthogonal fields 


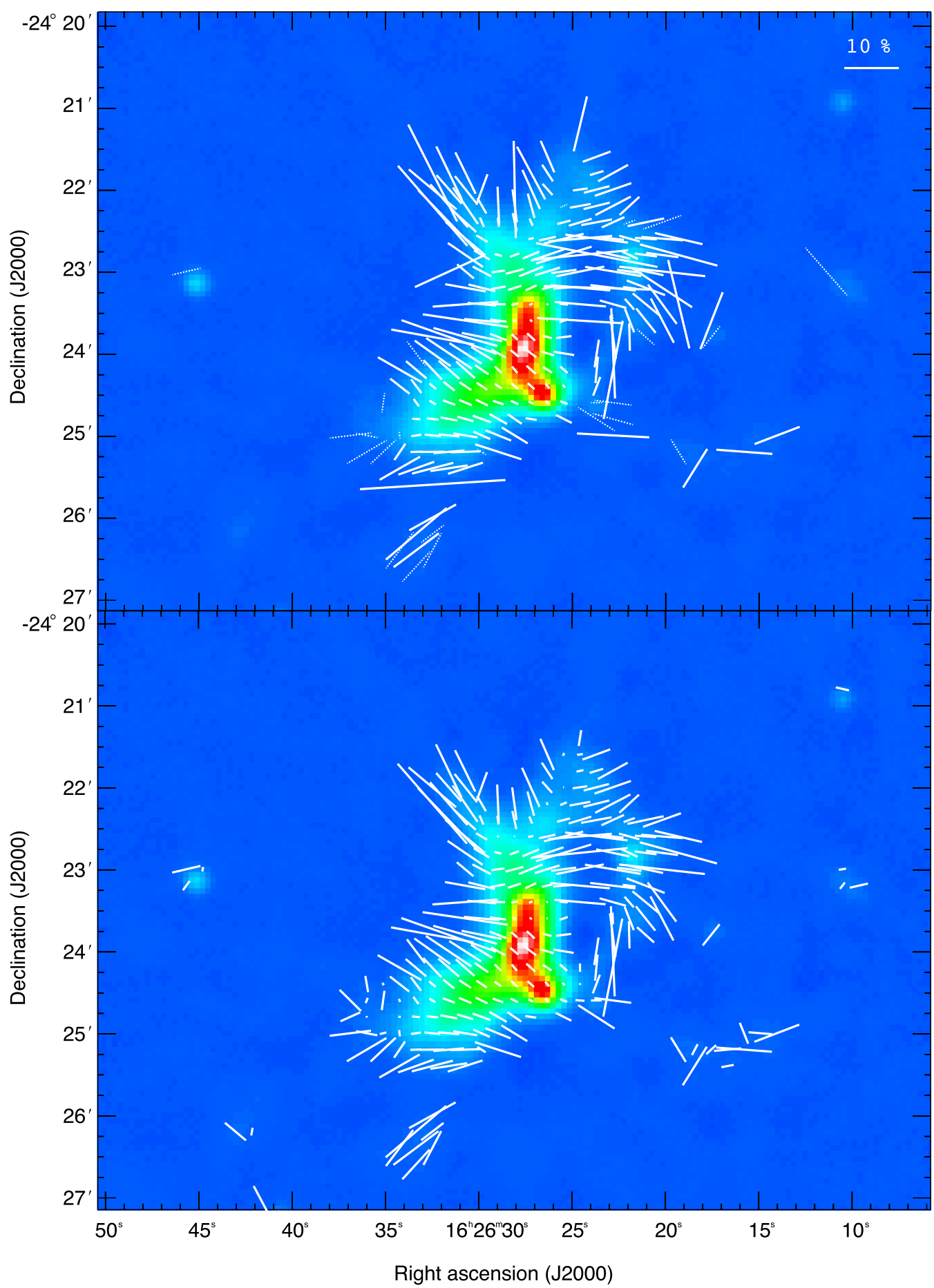

Figure 6. The $850 \mu \mathrm{m}$ polarization vector maps sampled on a $12^{\prime \prime}$ grid and rotated by $90^{\circ}$ (i.e., as in Figure 3 but rotated by $90^{\circ}$ ). The $90^{\circ}$ rotated vectors, which show the inferred magnetic field orientation projected on the plane of the sky, are plotted where $I>0, P / \delta P>2$, and $\delta P<4 \%$ (dotted vectors) and $I>0, P / \delta P>3$, and $\delta P<4 \%$ (solid vectors) in the top panel and where $I>0$ and $I / \delta I>20$ in the bottom panel. A $10 \%$ scale vector is shown in the upper right corner. See the text for these two panels with different selections $(P / \delta P$ and $I / \delta I)$.

are often seen in the densest parts of the cloud or cloud cores (Tamura et al. 1987, 1988; Nagai et al. 1998; André et al. 2013; Palmeirim et al. 2013; Matthews et al. 2014; Fissel et al. 2016; Planck Collaboration et al. 2016).

There are other local structures besides the $50^{\circ}$ component. To the north of A-MM 6 (component c), the magnetic field direction is almost north-south, and to the north of A-MM 7, the magnetic field direction is almost north-east (component b), which is the same as the direction of the northeast filament. Notable are the low degree of polarization near SM1N and some deviation in magnetic field direction near VLA 1623 and its outflow region.

In this paper, we have assumed that the $850 \mu \mathrm{m}$ emission measured by SCUBA-2 is dominated by the thermal dust continuum emission. However, the continuum emission can be contaminated by CO (3-2) line emission (Drabek et al. 2012). 


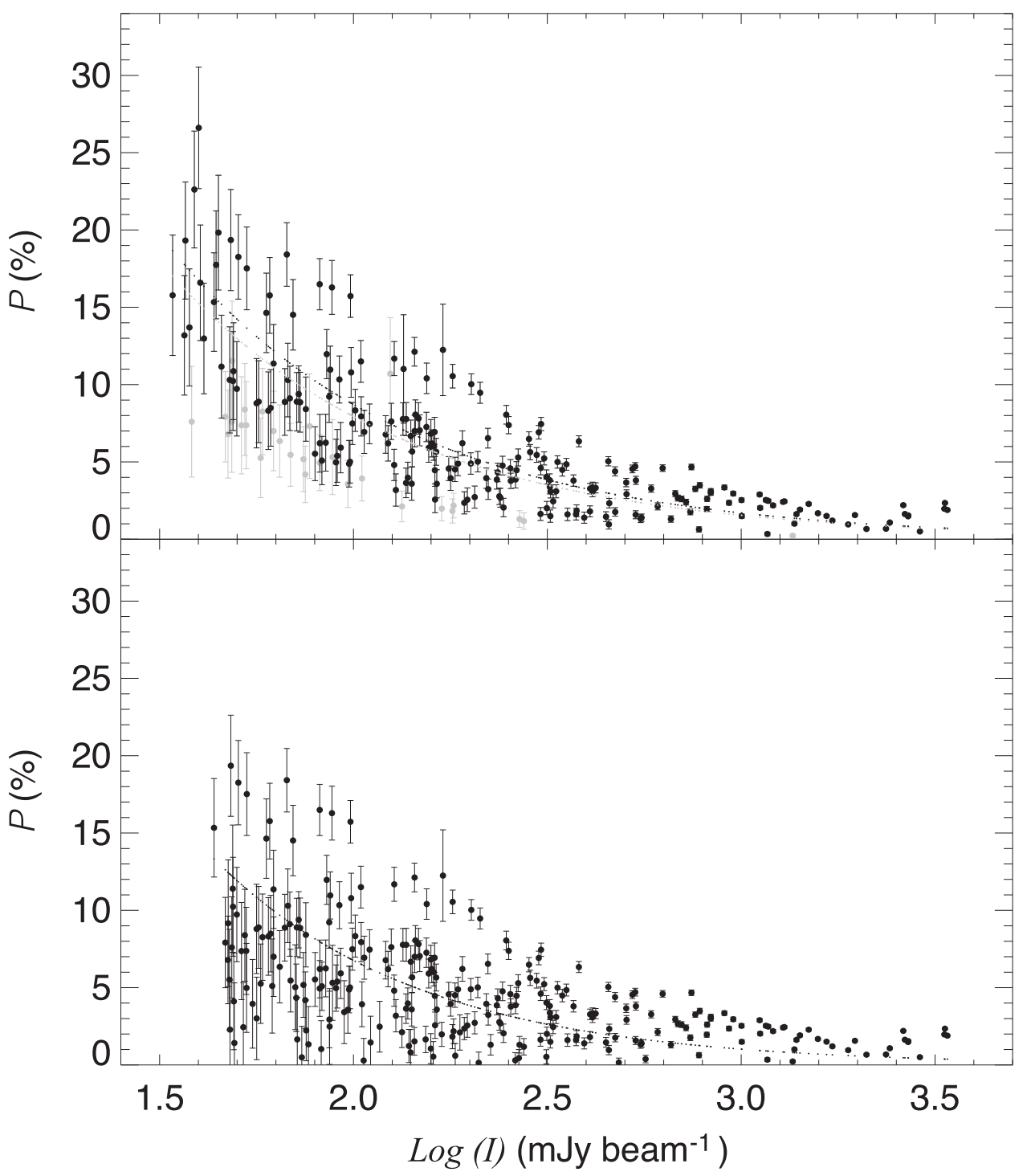

Figure 7. Degree of polarization $(P)$ vs. intensity $(I)$. Top panel: black circles show the sources with $I>0, P / \delta P>3$, and $\delta P<4 \%$, and gray circles show the sources with $I>0, P / \delta P>2$, and $\delta P<4 \%$. Bottom panel: black circles show the sources with $I>0, I / \delta I>20$, and $P>0$. Each least-squares-fit power law is shown as a dotted curve.

Figure 9 shows an overlay of the $\mathrm{CO}(3-2)$ line emission from White et al. (2015) on the $850 \mu \mathrm{m}$ continuum map. In the dense center of Oph-A, the $\mathrm{CO}$ contamination fraction is typically $<1 \%$. However, in the brightest regions of $\mathrm{CO}$ emission from the outflow from VLA 1623, the contamination fraction can be much higher (Pattle et al. 2015). The regions that have high CO contamination have very low column density values and are mostly along the jet axis between Oph-B and Oph-C/E/F, outside our field of view. In the dense center of Oph-A, the fractional contribution of $\mathrm{CO}$ is $<1 \%$ and generally does not exceed $10 \%$ anywhere on source. The ring-shaped region seen in our Stokes $I$ image to the west of Oph-A is dominated by the $\mathrm{CO}$ emission rather than the thermal dust emission. Since the $\mathrm{CO}$ emission is weak toward the bright dust emission $(<5 \%)$, even if it is polarized by the Goldreich-Kylafis effect, it will contribute minimally to our results.

\subsection{Local Magnetic Field Strength}

Polarization arising from dust grains, which are aligned with their major axes perpendicular to the magnetic field (e.g.,
Hoang \& Lazarian 2009), allows us to estimate the magnetic field direction. However, the present uncertainties in theories of dust grain alignment limit the ability of current techniques to trace magnetic fields without ambiguities (see Lazarian 2007; Lazarian et al. 2015 for a review).

The most common method to infer the magnetic field strength from polarized dust emission is the Davis-Chandrasekhar-Fermi method (more commonly referred as the Chandrasekhar-Fermi (CF) method; Davis 1951; Chandrasekhar \& Fermi 1953; see also Houde et al. 2016 and Pattle et al. 2017). The CF method infers the magnetic field strength by statistically comparing the dispersion in the polarization orientation with the dispersion in velocity. Therefore, the magnetic field strength projected on the plane of the sky can be calculated by

$$
B_{p}=\mathcal{Q} \sqrt{4 \pi \rho} \frac{\delta v_{\text {los }}}{\delta \theta}
$$

assuming that velocity perturbations are isotropic (Ostriker et al. 2001). In Equation (7), $\mathcal{Q}$ is a factor to account for various 


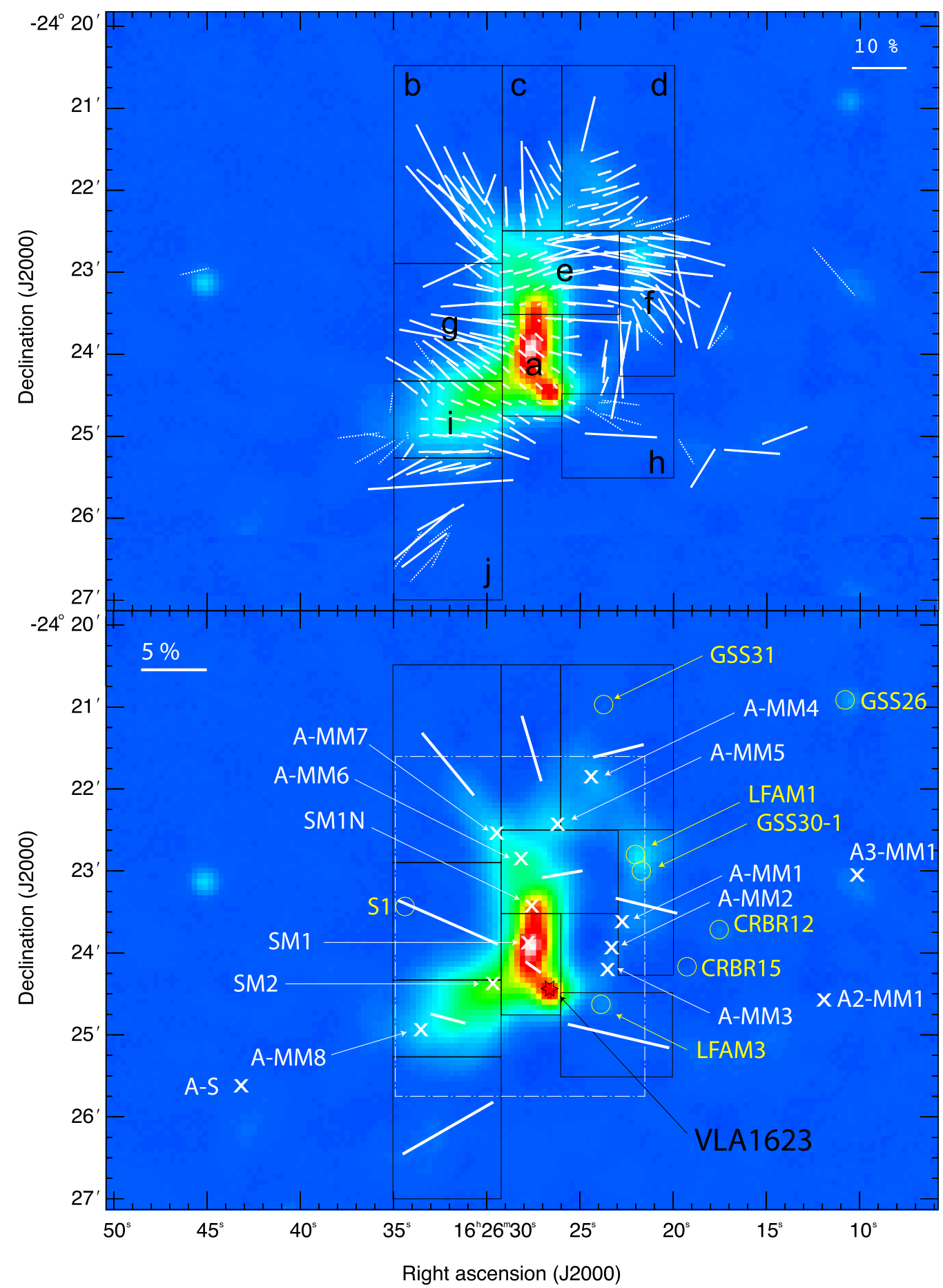

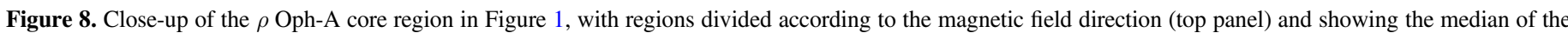

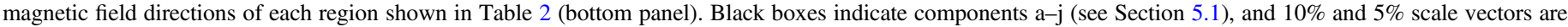

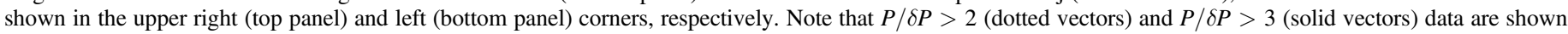

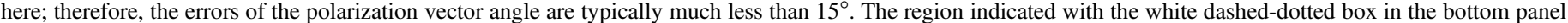
corresponds to the region shown in Figure 10.

averaging effects (see Crutcher et al. 2004 and Houde 2004 for details), $\rho$ is the mean density of the cloud, $\delta v_{\text {los }}$ is the rms lineof-sight velocity, and $\delta \theta$ is the dispersion in the polarization angle. To estimate the magnetic field strength in the $\rho$ Oph-A core region, a correction factor of $\mathcal{Q}=0.5$ is adopted here because the magnetic field appears to be ordered (Ostriker et al. 2001; Houde 2004; also see Falceta-Gonçalves et al. 2008;
Novak et al. 2009; Pattle et al. 2017). Since we apply this formula only to the subregions where the angle dispersion is relatively small $\left(\leqslant 25^{\circ}\right)$, and the velocity dispersion of the molecular lines tracing high-density regions is available in the next paragraph, $\mathcal{Q}=0.5$ is appropriate, as simulated by Ostriker et al. (2001). Note that if the turbulence correlation length is not resolved and therefore their simulation assumption 
Table 2

Median $P$, Median $\theta$, and Magnetic Field Direction in Each Component from Figure 8

\begin{tabular}{lccr}
\hline \hline Component & $\begin{array}{c}P \\
(\%)\end{array}$ & $\begin{array}{c}\theta \\
(\mathrm{deg})\end{array}$ & $\begin{array}{c}\text { MF Direction } \\
\text { a } \\
(\mathrm{deg})\end{array}$ \\
\hline $\mathrm{a}$ & $1.44 \pm 0.07$ & $144.4 \pm 1.5$ & 54.4 \\
$\mathrm{~b}$ & $6.18 \pm 0.95$ & $129.7 \pm 4.4$ & 39.7 \\
$\mathrm{c}$ & $5.19 \pm 0.95$ & $106.5 \pm 5.2$ & 16.5 \\
$\mathrm{~d}$ & $3.96 \pm 0.80$ & $14.0 \pm 5.8$ & 104.0 \\
$\mathrm{e}$ & $3.12 \pm 0.29$ & $9.6 \pm 2.7$ & 99.6 \\
$\mathrm{f}$ & $4.83 \pm 0.79$ & $166.2 \pm 4.7$ & 76.2 \\
$\mathrm{~g}$ & $8.28 \pm 0.97$ & $156.3 \pm 3.3$ & 66.3 \\
$\mathrm{~h}$ & $7.92 \pm 2.82$ & $166.9 \pm 10.2$ & 76.9 \\
$\mathrm{i}$ & $2.76 \pm 0.29$ & $165.0 \pm 3.0$ & 75.0 \\
$\mathrm{j}$ & $7.96 \pm 2.23$ & $29.9 \pm 8.0$ & 119.9 \\
\hline
\end{tabular}

Note. Median polarization degrees $P$ and polarization position angles $\theta$ were calculated using Stokes $Q$ and $U$ in each component of Figure 8 .

${ }^{a} \mathrm{MF}$ : Indicated magnetic field direction, which is $\theta$ rotated by $90^{\circ}$.

is not valid, the case of the $\mathcal{Q}$ factor can be much lower (Heitsch et al. 2001; see also Houde et al. 2009). Then Equation (7) can be expressed as follows (Lai et al. 2002):

$$
B_{p}=8.5 \frac{\sqrt{n_{\mathrm{H} 2} /\left(10^{6} \mathrm{~cm}^{-3}\right)} \Delta \mathrm{v} /\left(\mathrm{km} \mathrm{s}^{-1}\right)}{\delta \theta\left(^{\circ}\right)} \mathrm{mG} .
$$

Here $n_{\mathrm{H}_{2}}$ is the number density of hydrogen molecules and $\Delta v$ is the line width.

As mentioned in the previous section, there are several magnetic field components in the $\rho$ Oph-A core region. Since they are different from each other either in direction or degree of polarization, we estimate the magnetic field strength of each component separately. To investigate their magnetic field strengths individually, we estimated the median polarization position angles, which indicate the local average magnetic field directions of each component. Table 2 shows the median degrees of polarizations and position angles calculated using Stokes $Q$ and $U$ in each region. Figure 8 shows the vectors in each region averaged over each region.

The local average density in each of components $a-j$ is calculated from our Stokes $I$ data, assuming that the core depth is equal to the geometric mean size of each subcore where the polarization data exist and ranges from $2 \times 10^{6}$ to $7 \times 10^{4} \mathrm{~cm}^{-3}$. Since the $\rho$ Oph-A core has a complex magnetic field structure showing various directions in each subcore, we do not attempt to apply to the CF method to the entire core, but only to the subcores showing a relatively well-defined magnetic field direction. In components a, d, and e, André et al. (2007) estimated a velocity dispersion of $0.26,0.15$, and $0.17 \mathrm{~km} \mathrm{~s}^{-1}$, respectively. These are nonthermal line dispersions from $\mathrm{N}_{2} \mathrm{H}^{+}$ (1-0) observations. Using these values with the standard deviation in field direction of $1.5,5^{\circ} .8$, and 2.7 found in each region, the magnetic field strength projected on the plane of the sky is calculated as $B p \sim 5,0.2$, and $0.8 \mathrm{mG}$ (cf. Table 2). The estimated magnetic field strength in the $\rho$ Oph-A core region is larger than that in other molecular clouds derived using the $\mathrm{CF}$ method (namely, 20-200 $\mu \mathrm{G}$; Andersson \& Potter 2005; Poidevin \& Bastien 2006; Alves et al. 2008; Kwon et al. 2010, 2011; Sugitani et al. 2011; Kusune et al. 2015) but comparable to that in the Orion A region (see, e.g., Pattle et al. 2017). Our high magnetic field strengths may be attributed to using the higher $\mathrm{H}_{2}$ densities associated with the subcores rather than the lower $\mathrm{H}_{2}$ densities associated with the larger $\rho$ Oph-A core. Thus, we conservatively take these field strengths as an orderof-magnitude estimate. These values are still representative of the field strength toward the subcores in $\rho$ Oph-A and can be taken as an upper limit for the surrounding gas.

Finally, it should be noted that there are certain limitations in the $\mathrm{CF}$ technique, such as the effect of the limited telescope resolution (Heitsch et al. 2001). Also note that our estimates are only for some subregions where the field dispersions are relatively small. Therefore, both of these effects tend to bias toward a high magnetic field strength. In addition, more sophisticated applications of the $\mathrm{CF}$ technique, such as those described in Hildebrand et al. (2009) or Pattle et al. (2017), would be desirable in future works.

\subsection{Magnetic Fields and Centroid Velocity}

Our polarimetric data will be useful to discuss the correlation between the magnetic field and the velocity field in each core. However, this is beyond the scope of this first-look paper. Therefore, in this section, we show an example of a possible correlation between magnetic field and velocity gradient.

Strong Alfvénic turbulence develops eddy-like motions perpendicular to the local magnetic field direction (Goldreich \& Sridhar 1995). Very recently, González-Casanova \& Lazarian (2017) proposed that this fact can be used to study the direction of magnetic fields by using the velocity gradient calculated from the centroid velocity. The centroid velocity is an intensity-weighted average velocity along the line of sight (e.g., Miesch et al. 1999). Here we try to compare the magnetic field direction in the $\rho$ Oph-A core region with the centroid velocity components.

André et al. (2007) measured subsonic or transonic levels of internal turbulence within the condensations, and their result supports the view that most of the L1688 starless condensations are gravitationally bound and prestellar in nature. Figure 10 shows a comparison between the magnetic field direction (this work) and the centroid velocity components of $\mathrm{N}_{2} \mathrm{H}^{+}(1-0)$ spectra (André et al. 2007). The apparent main velocity core gradient (indicated by arrows in Figure 10) appears to be roughly perpendicular to the magnetic field orientation traced by POL-2. Certainly, these observations should be compared with theoretical modeling using the physical parameters of the $\rho$ Oph-A core in future.

\subsection{Tracing Magnetic Fields across Different Wavelengths}

Polarimetry in the $\rho$ Oph-A core region was reported previously by several authors at other wavelengths. Sato et al. (1988) carried out near-infrared polarimetry (in the $K$ band only) of 20 sources that are embedded within the densest region of the $\rho$ Oph dark cloud with a single-channel detector, and they suggested that there are three dominant components of the polarization position angles $0^{\circ}, 50^{\circ}$, and $150^{\circ}$. Recently, Kwon et al. (2015) presented wide and deep near-infrared polarimetry (in the $J H K_{s}$ bands) of the $\rho$ Oph regions, which corresponds to the densest part of L1688. Since they cover a wider region than our observations (but much more sparsely due to the limited number of stars available for the aperture polarimetry), we compare their polarimetry data covering a $40^{\prime} \times 40^{\prime}$ region with our submillimeter data. In this active cluster-forming region, they found that the magnetic fields appear to be connected from core to core, rather than as a simple overlap of 


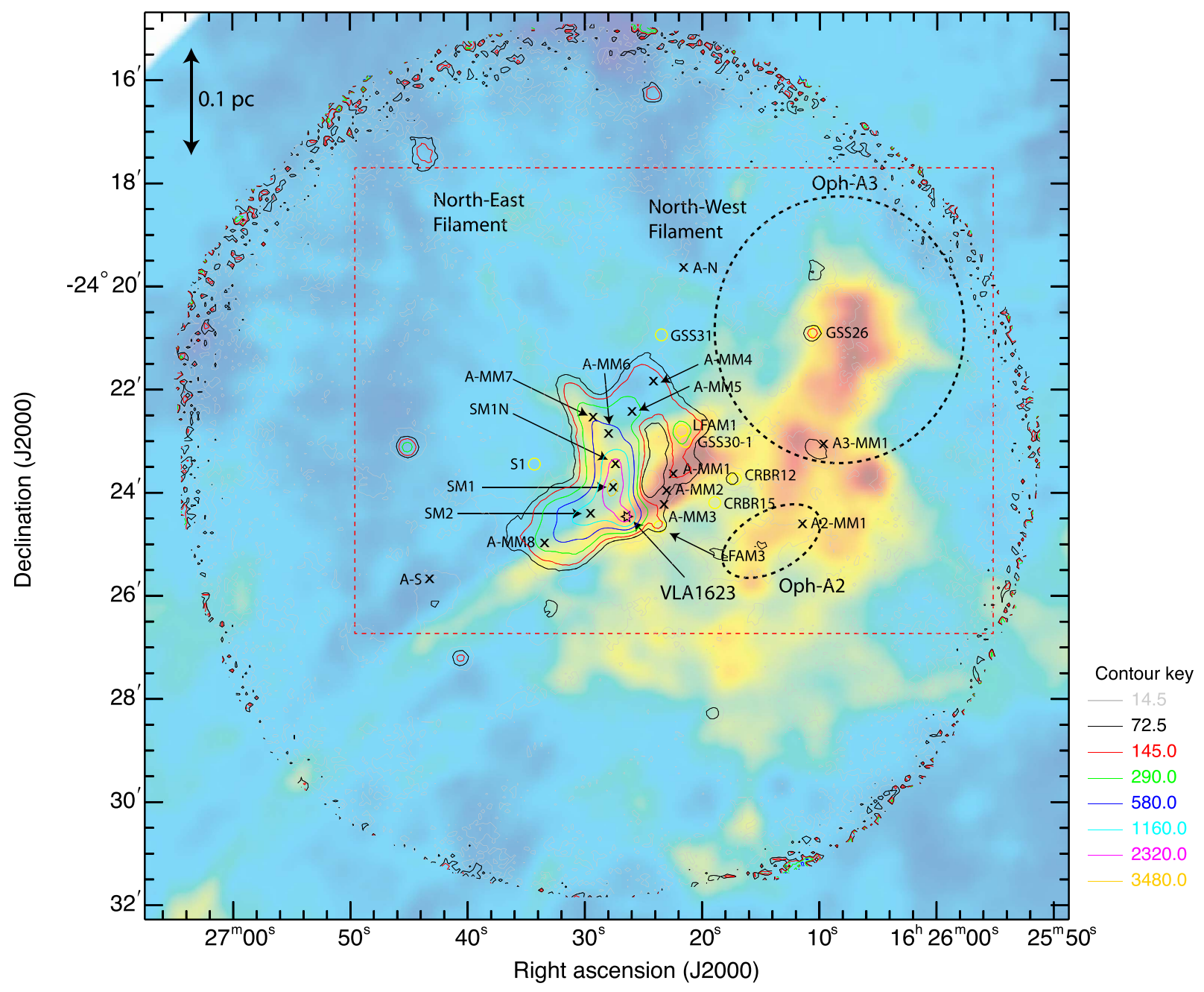

Figure 9. Contours of the $850 \mu \mathrm{m}$ total intensity (Stokes $I$ ) image (cf. Figure 1 of this work) of the $\rho$ Oph-A field superimposed on HARP CO $J=3-2$ observations showing the integrated emission between -5 and $+12 \mathrm{~km} \mathrm{~s}^{-1}$ (cf. Figure 1 of White et al. 2015).

the different cloud core components. Putting it differently, the magnetic field morphology seems to be connected between different cores in the $\rho$ Oph molecular cloud complex. In addition, comparing their near-infrared polarimetric results with the large-scale magnetic field structures obtained from a previous optical polarimetric study (Vrba et al. 1976), they suggested that the magnetic field structures in the $\rho$ Oph core were distorted by the cluster formation in this region, which may have been induced by shock compression due to wind/ radiation from the Scorpius-Centaurus association. Also note that there is $350 \mu \mathrm{m}$ submillimeter polarization from the CSO in Dotson et al. (2010) for $\rho$ Oph-A. Their data are broadly consistent with our $850 \mu \mathrm{m}$ map.

Our new submillimeter polarimetry demonstrates that one of the main polarization position angles in Oph-A is approximately $50^{\circ}$ (see Figures 3-10) and so is well aligned with the $50^{\circ}$ magnetic field found in the near-infrared (Kwon et al. 2015; see also Figure 5 in this paper for the comparison within the same field of view). Kwon et al. (2015) found that the " $50^{\circ}$ component" is the dominant magnetic field component in the observed region; it can be seen as a distinct clump in the diagram plotting degree of polarization versus polarization angle (Figure 9 of Kwon et al. 2015) and in the histogram of polarization position angles (Figure 10 of Kwon et al. 2015).
This component is seen in the northeast regions of $\rho$ Oph-A (and in $\rho$ Oph-B and $\rho$ Oph-E on a large scale, regions not covered in this work). The " 0 ' component" can be seen from $\rho$ Oph-A toward $\rho$ Oph-AC (located at the southeastern region of $\rho$ Oph-A, which is not shown in our submillimeter map; cf. Kwon et al. 2015). In contrast, in Oph-A, both the $0^{\circ}$ and the $50^{\circ}$ components exist.

Figure 11 shows the histogram of polarization position angles for the $90^{\circ}$ rotated submillimeter polarization vectors, as well as for the $H$-band polarization position angles from Kwon et al. (2015). The distribution is relatively widespread, but if we refer to both the $H$-band polarization vector map (Figure 8 of Kwon et al. 2015) and this histogram, we see several components, of which the components at $0^{\circ}$ and $50^{\circ}$ are most clearly seen. As shown in Figure 11, the distribution of the polarization position angles obtained from submillimeter polarimetry is in relatively good agreement with that obtained from near-infrared polarimetry for the $0^{\circ}$ and $50^{\circ}$ components but not for the $150^{\circ}$ component. Note that since our submillimeter map covers a small part of the area covered by the near-infrared polarimetry survey and we see much higher column density regions of $\rho$ Oph-A, there is also some inconsistency between the distributions of submillimeter and near-infrared polarization angles. Therefore, our results indicate 


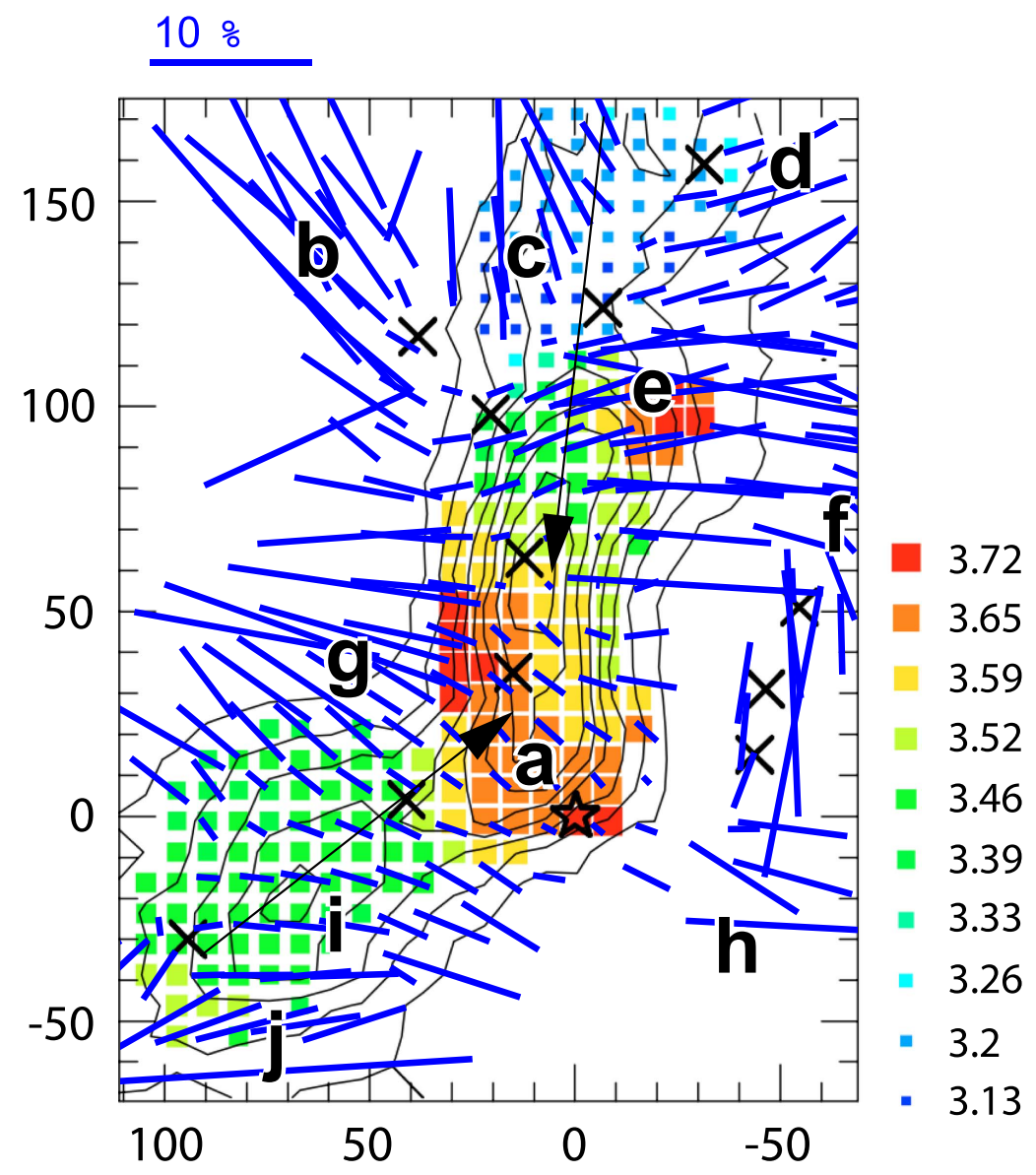

Figure 10. Comparison between magnetic field directions from this work and the centroid velocity components of $\mathrm{N}_{2} \mathrm{H}^{+}(1-0)$ spectra (filled squares of varying sizes and colors; Figure 6(c) of André et al. 2007). The (0, 0) offset corresponds to the position $\alpha=16^{\mathrm{h}} 26^{\mathrm{m}} 26^{\mathrm{s}} .45, \delta=-24^{\circ} 24^{\prime} 30^{\prime \prime} 8$ [J2000]. The contours, which were drawn by André et al. (2007), go from 2 to $16 \mathrm{~K} \mathrm{~km} \mathrm{~s}^{-1}$. The color code shows the velocity centroid. The underlying contours represent the same $\mathrm{N}_{2} \mathrm{H}^{+}(1-0)$ integrated intensity maps. Our suggested centroid velocity gradient is shown by black arrows, which are not in the original figure (André et al. 2007). Shown in the upper left is the $10 \%$ scale vector for the $90^{\circ}$ rotated submillimeter polarization vectors. The letters a-j are the magnetic field components defined from our submillimeter polarimetry (see text). Crosses mark the $1.2 \mathrm{~mm}$ continuum positions of starless condensations, while a star marks the position of VLA 1623 (see André et al. 2007). The labels $a-j$ indicate the distinct magnetic field components in each subregion $a-j$.

that both submillimeter emission polarization and near-infrared dichroic polarization may trace the magnetic field structures associated with the $\rho$ Oph-A core region at different spatial scales and regions along the line of sight.

Previous observations have shown agreement between the magnetic field structures seen at various wavelengths, such as near- and far-infrared or submillimeter wavelengths (e.g., Tamura et al. 1996, 2007; Kandori et al. 2007; Kwon et al. 2011). Our new results are consistent with this behavior, although the greatest-density regions can be traced only by submillimeter polarimetry. The $50^{\circ}$ component seen in the lower-density regions of the submillimeter map around the edge of the core (the northeast filament; cf. Figure 1) is consistent with the $50^{\circ}$ component seen in the lower-density tracer of near-infrared polarization (Kwon et al. 2015), giving us still further confidence in our observations. Our data are also consistent with the recently released HAWC + data taken by SOFIA (Santos et al. 2018). A combination of polarimetric observations over wavelengths and scales observed by instruments such as ALMA and by $8 \mathrm{~m}$ class optical/infrared telescopes will become more important in the future to test the range of scales over which this behavior holds.

\section{Summary}

In this paper, we present the first-look analysis for the $\rho$ Oph-A SCUBA-2/POL-2 continuum map observed by the JCMT Gould Belt polarization survey at $850 \mu \mathrm{m}$. The $\rho$ Oph molecular cloud complex is one of the nearest laboratories for examining active star formation sites, offering a wealth of objects to aid in a better understanding of the dominant physical processes present in the region. The SCUBA-2/POL2 polarimeter is a very powerful instrument to trace the magnetic field structure in star-forming regions such as the Oph molecular cloud complex. The main results are as follows.

1. We have identified at least 10 magnetic field components in the $\rho$ Oph-A core region whose position angles and degrees of polarization are distinct from each other. However, some of them can be part of a coherent structure. Our polarimetric results are not only consistent with previous results in the bright core regions but also reveal the fields in the outer regions for the first time. These components represent the magnetic fields of the subcores identified as local continuum intensity peaks or distinct velocity structures within the Oph-A core; they 


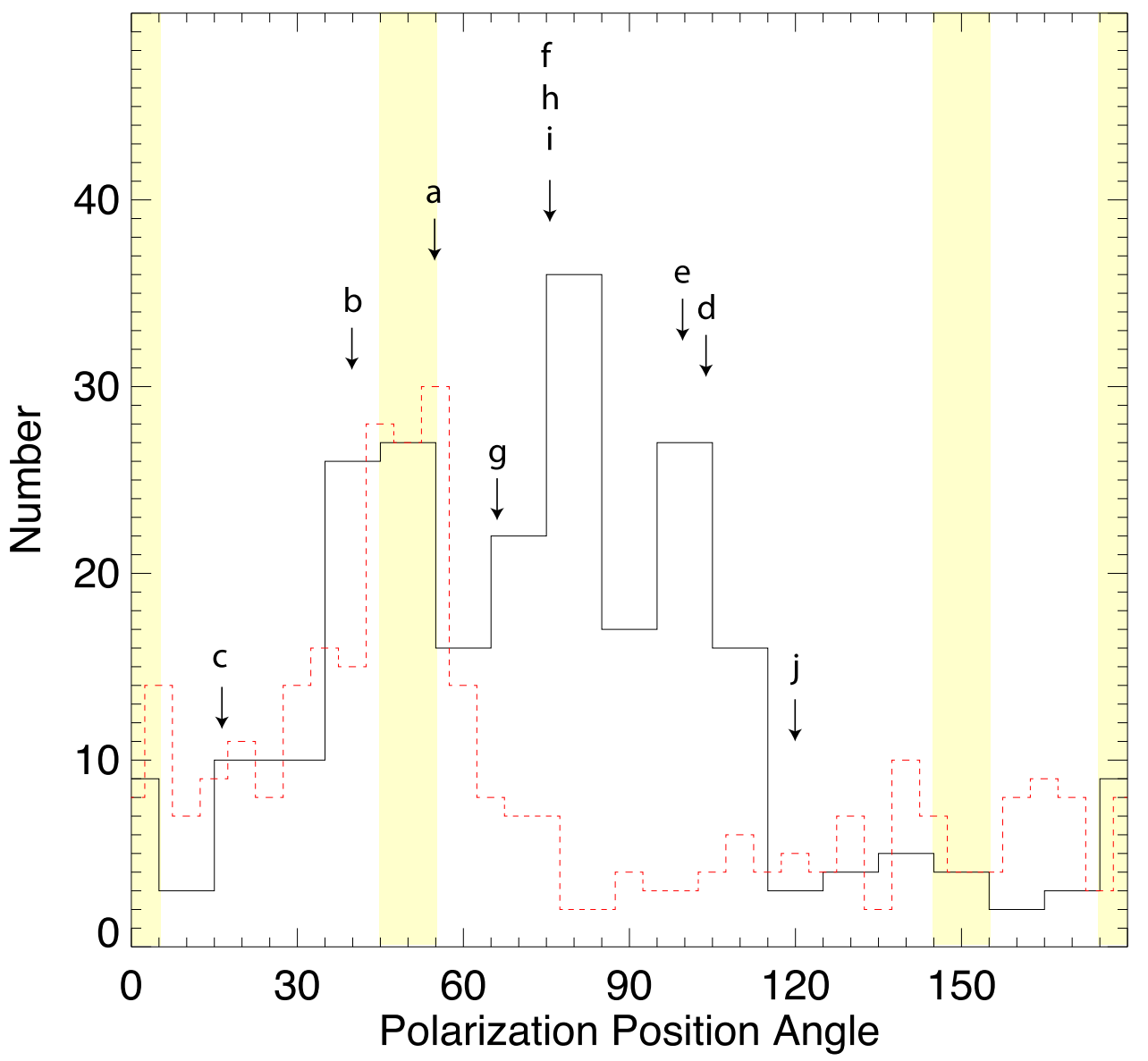

Figure 11. Histogram of polarization position angles for the $90^{\circ}$ rotated submillimeter vectors with $I>0, P / \delta P>2$, and $\delta P<4 \%$ (black). Black line: $90^{\circ}$ rotated submillimeter polarization vectors (this work). Note that a $10^{\circ}$ bin is used. Red dotted line: $H$-band polarization position angles (Kwon et al. 2015). Black arrows indicate the magnetic field components from the submillimeter data. The labels a-j indicate the average magnetic field direction of the distinct magnetic field components a-j projected on the plane of the sky. Yellow shaded regions indicate the major magnetic field directions suggested from the previous near-infrared data.

show a large variation even within the small (approximately $0.2 \mathrm{pc}$ ) region observed.

2. The dominant component of the magnetic field over $\rho$ Oph-A is the $50^{\circ}$ component. This direction is consistent with that inferred from the near-infrared polarimetry of the $\rho$ Oph cloud core.

3. Although the average direction of the main component is approximately $50^{\circ}$, the magnetic field tends to be locally perpendicular (approximately $100^{\circ}-110^{\circ}$ ) to the arcstructure. Between SM1N and A-MM 6, the field direction is almost east-west, while the arc extends to the northeast or northwest and the field direction extends toward LFAM 1 and GSS 30-1. The perpendicularity between the core shape and the magnetic field direction may be important in understanding the origin and formation of this core. Such perpendicularity is often seen in the densest parts of clouds and cloud cores.

4. There are local structures besides the $50^{\circ}$ component. To the north of A-MM 6, the field direction is almost northsouth, and to the north of A-MM 7, the field direction is almost north-east, which is the same as the direction of the northeast filament. Notable are the low degree of polarization near SM1N and some deviation in field direction near VLA 1623 and its outflow region.

5. Using the CF method, we roughly estimate the strengths of the magnetic field projected on the plane of the sky in several subcore regions to be up to a few $\mathrm{mG}$.
6. We have found that the main large-scale core velocity gradient is approximately perpendicular to the inferred cloud magnetic field orientation.

We thank the referee for thorough and insightful comments, which improved the paper significantly. The James Clerk Maxwell Telescope is operated by the East Asian Observatory on behalf of the National Astronomical Observatory of Japan, the Academia Sinica Institute of Astronomy and Astrophysics, the Korea Astronomy and Space Science Institute, the National Astronomical Observatories of China, and the Chinese Academy of Sciences (grant No. XDB09000000), with additional funding support from the Science and Technology Facilities Council of the United Kingdom and participating universities in the United Kingdom and Canada. The James Clerk Maxwell Telescope has historically been operated by the Joint Astronomy Centre on behalf of the Science and Technology Facilities Council of the United Kingdom, the National Research Council of Canada, and the Netherlands Organisation for Scientific Research. Additional funds for the construction of SCUBA-2 and POL-2 were provided by the Canada Foundation for Innovation. The data taken in this paper were observed under the project code M16AL004. Data analysis was in part carried out on the open-use data analysis computer system at the Astronomy Data Center (ADC) of the National Astronomical Observatory of Japan. J.K. was supported by MEXT KAKENHI grant number $16 \mathrm{H} 07479$ and the Astrobiology Center of NINS. M.T. was supported by MEXT 
KAKENHI grant number 22000005. D.W.T. and K.P. acknowledge Science and Technology Facilities Council (STFC) support under grant numbers ST/K002023/1 and ST/M000877/1. K.P. was an International Research Fellow of the Japan Society for the Promotion of Science. K.P. and S.P.L. acknowledge the support of the Ministry of Science and Technology of Taiwan (Grant No. 106-2119-M-007-021-MY3). M.K. was supported by the Basic Science Research Program through the National Research Foundation of Korea (NRF) funded by the Ministry of Science, ICT \& Future Planning (No. NRF-2015R1C1A1A01052160). C.W.L. was supported by the Basic Science Research Program though the NRF funded by the Ministry of Education, Science, and Technology (NRF-2016R1A2B4012593). W.K. was supported by the Basic Science Research Program through the NRF (NRF-2016R1C1B2013642). J.E.L. is supported by the Basic Science Research Program through the National Research Foundation of Korea (grant No. NRF-2018R1A2B6003423) and the Korea Astronomy and Space Science Institute under the R\&D program supervised by the Ministry of Science, ICT and Future Planning. T.L. is supported by a KASI fellowship and an EACOA fellowship. Team BISTRO-J is in part financially supported by 260 individuals.

Facility: James Clerk Maxwell Telescope (JCMT).

Software: Starlink (Currie et al. 2014), smurf (Berry et al. 2005; Chapin et al. 2013).

\section{ORCID iDs}

Jungmi Kwon (iD https://orcid.org/0000-0003-2815-7774 Motohide Tamura (iD https://orcid.org/0000-0002-6510-0681 Masafumi Matsumura (i) https://orcid.org/0000-0002-6906-0103 Kate Pattle (1) https://orcid.org/0000-0002-8557-3582 Sarah Sadavoy (i) https://orcid.org/0000-0001-7474-6874 Brenda C. Matthews (10) https://orcid.org/0000-00033017-9577

Derek Ward-Thompson (iD https://orcid.org/0000-0003-1140-2761 Ray S. Furuya (1) https://orcid.org/0000-0003-0646-8782 Andy Pon (10) https://orcid.org/0000-0003-4612-1812 Koji S. Kawabata (i) https://orcid.org/0000-0001-6099-9539 Takashi Onaka (D) https://orcid.org/0000-0002-8234-6747 Miju Kang (i) https://orcid.org/0000-0002-5016-050X Thiem Hoang (iD https://orcid.org/0000-0003-2017-0982 Chang Won Lee (i) https://orcid.org/0000-0002-3179-6334 Sang-Sung Lee (i) https://orcid.org/0000-0002-6269-594X Tie Liu (10 https://orcid.org/0000-0002-5286-2564

Shu-ichiro Inutsuka (iD https://orcid.org/0000-0003-4366-6518 Chakali Eswaraiah (i) https://orcid.org/0000-0003-4761-6139 Pierre Bastien (ib https://orcid.org/0000-0002-0794-3859 Woojin Kwon (iD https://orcid.org/0000-0003-4022-4132 Shih-Ping Lai (i) https://orcid.org/0000-0001-5522-486X Keping Qiu (iD https://orcid.org/0000-0002-5093-5088 Simon Coudé (ib https://orcid.org/0000-0002-0859-0805 Sarah F. Graves (1) https://orcid.org/0000-0001-9361-5781 Jane S. Greaves (D) https://orcid.org/0000-0002-3133-413X Martin Houde (1D https://orcid.org/0000-0003-4420-8674 Doug Johnstone (i) https://orcid.org/0000-0002-6773-459X Jason M. Kirk (i) https://orcid.org/0000-0002-4552-7477 Patrick M. Koch (iD https://orcid.org/0000-0003-2777-5861 Di Li ib https://orcid.org/0000-0003-3010-7661

Harriet Parsons (iD https://orcid.org/0000-0002-6327-3423 Ramprasad Rao (10) https://orcid.org/0000-0002-1407-7944 Hiroko Shinnaga (ib https://orcid.org/0000-0001-9407-6775 Sven van Loo (1D https://orcid.org/0000-0003-4746-8500
Yusuke Aso (D) https://orcid.org/0000-0002-8238-7709 Do-Young Byun (iD https://orcid.org/0000-0003-1157-4109 Huei-Ru Chen (iD https://orcid.org/0000-0002-9774-1846 Wen Ping Chen (iD https://orcid.org/0000-0003-0262-272X Jungyeon Cho (ib https://orcid.org/0000-0003-1725-4376 Antonio Chrysostomou (iD https://orcid.org/0000-00029583-8644

Eun Jung Chung (iD https://orcid.org/0000-0003-0014-1527 Stewart P. S. Eyres (i) https://orcid.org/0000-0002-6663-7675 Rachel K. Friesen (iD https://orcid.org/0000-0001-7594-8128 Gary Fuller (iD https://orcid.org/0000-0001-8509-1818 Tim Gledhill (i) https://orcid.org/0000-0002-2859-4600 Jennifer Hatchell (i) https://orcid.org/0000-0002-4870-2760 Ji-hyun Kang (i) https://orcid.org/0000-0001-7379-6263 Sung-ju Kang (i) https://orcid.org/0000-0002-5004-7216 Francisca Kemper (10 https://orcid.org/0000-0003-2743-8240 Kee-Tae Kim (D) https://orcid.org/0000-0003-2412-7092 Kyoung Hee Kim (iD https://orcid.org/0000-0001-9597-7196 Mi-Ryang Kim (1D https://orcid.org/0000-0002-1408-7747 Kevin M. Lacaille (i) https://orcid.org/0000-0001-9870-5663 Jeong-Eun Lee (iD https://orcid.org/0000-0003-3119-2087 Hua-bai Li (i) https://orcid.org/0000-0003-2641-9240 Sheng-Yuan Liu (i) https://orcid.org/0000-0003-4603-7119 A-Ran Lyo (ib https://orcid.org/0000-0002-9907-8427 Steve Mairs (ib https://orcid.org/0000-0002-6956-0730 Fumitaka Nakamura (i) https://orcid.org/0000-00015431-2294

Nagayoshi Ohashi (i) https://orcid.org/0000-0003-0998-5064 Tae-Soo Pyo (iD https://orcid.org/0000-0002-3273-0804 Lei Qian (i) https://orcid.org/0000-0003-0597-0957 John Richer (1) https://orcid.org/0000-0002-9693-6860 Anna M. M. Scaife (i) https://orcid.org/0000-0002-5364-2301 Archana Soam (1D https://orcid.org/0000-0002-6386-2906 Ya-Wen Tang (iD https://orcid.org/0000-0002-0675-276X Kohji Tomisaka (1) https://orcid.org/0000-0003-2726-0892 Hongchi Wang (iD https://orcid.org/0000-0003-0746-7968 Jia-Wei Wang (iD https://orcid.org/0000-0002-6668-974X Hsi-Wei Yen (iD https://orcid.org/0000-0003-1412-893X Jinghua Yuan (iD https://orcid.org/0000-0001-8060-3538 Chuan-Peng Zhang (iD https://orcid.org/0000-0002-4428-3183 Takao Nakagawa (D) https://orcid.org/0000-0002-6660-9375 Akimasa Kataoka (iD https://orcid.org/0000-0003-4562-4119 Masato I. N. Kobayashi (i) https://orcid.org/0000-00033990-1204

\section{References}

Alves, F. O., Franco, G. A. P., \& Girart, J. M. 2008, A\&A, 486, L13 Andersson, B.-G., \& Potter, S. B. 2005, MNRAS, 356, 1088

André, P., Belloche, A., Motte, F., \& Peretto, N. 2007, A\&A, 472, 519

André, P., Könyves, V., Arzoumanian, D., Palmeirim, P., \& Peretto, N. 2013, in ASP. Conf. Ser. 476, New Trends in Radio Astronomy in the ALMA Era (San Francisco, CA: ASP), 95

André, P., Men'shchikov, A., Bontemps, S., et al. 2010, A\&A, 518, L102 Andre, P., Ward-Thompson, D., \& Barsony, M. 1993, ApJ, 406, 122 Bergin, E. A., \& Tafalla, M. 2007, ARA\&A, 45, 339

Berry, D. S., Gledhill, T. M., Greaves, J. S., \& Jenness, T. 2005, in ASP Conf. Ser. 343, Astronomical Polarimetry: Current Status and Future Directions, ed. A. Adamson et al. (San Francisco, CA: ASP), 71

Bontemps, S., \& Andre, P. 1997, in IAU Symp. 182, Low Mass Star Formation-from Infall to Outflow, ed. F. Malbet \& A. Castets, 63

Cashman, L. R., \& Clemens, D. P. 2014, ApJ, 793, 126

Chandrasekhar, S., \& Fermi, E. 1953, ApJ, 118, 113

Chapin, E. L., Berry, D. S., Gibb, A. G., et al. 2013, MNRAS, 430, 2545

Chen, X., Arce, H. G., Zhang, Q., et al. 2013, ApJ, 768, 110

Chini, R. 1981, A\&A, 99, 346 
Crutcher, R. M., Nutter, D. J., Ward-Thompson, D., \& Kirk, J. M. 2004, ApJ, 600,279

Currie, M. J., Berry, D. S., Jenness, T., et al. 2014, adass XXIII, 485, 391

Davis, L. 1951, PhRv, 81, 890

Davis, L., Jr., \& Greenstein, J. L. 1951, ApJ, 114, 206

de Geus, E. J., de Zeeuw, P. T., \& Lub, J. 1989, A\&A, 216, 44

Dempsey, J. T., Friberg, P., Jenness, T., et al. 2013, MNRAS, 430, 2534

Dent, W. R. F., Matthews, H. E., \& Walther, D. M. 1995, MNRAS, 277, 193

Di Francesco, J., André, P., \& Myers, P. C. 2004, ApJ, 617, 425

Dotson, J. L., Vaillancourt, J. E., Kirby, L., et al. 2010, ApJS, 186, 406

Drabek, E., Hatchell, J., Friberg, P., et al. 2012, MNRAS, 426, 23

Elmegreen, B. G. 2000, ApJ, 539, 342

Evans, N. J., II, Dunham, M. M., Jørgensen, J. K., et al. 2009, ApJS, 181, 321

Falceta-Gonçalves, D., Lazarian, A., \& Kowal, G. 2008, ApJ, 679, 537

Fissel, L. M., Ade, P. A. R., Angilè, F. E., et al. 2016, ApJ, 824, 134

Friberg, P., Bastien, P., Berry, D., et al. 2016, Proc. SPIE, 9914, 991403

Goldreich, P., \& Sridhar, S. 1995, ApJ, 438, 763

González-Casanova, D. F., \& Lazarian, A. 2017, ApJ, 835, 41

Goodman, A. A., Jones, T. J., Lada, E. A., \& Myers, P. C. 1992, ApJ, 399, 108

Greaves, J. S., Holland, W. S., Minchin, N. R., Murray, A. G., \& Stevens, J. A. 1999, A\&A, 344, 668

Hartmann, L., Ballesteros-Paredes, J., \& Bergin, E. A. 2001, ApJ, 562, 852

Heitsch, F., Zweibel, E. G., Mac Low, M.-M., Li, P., \& Norman, M. L. 2001, ApJ, 561, 800

Hildebrand, R. H., Kirby, L., Dotson, J. L., Houde, M., \& Vaillancourt, J. E. 2009, ApJ, 696, 567

Hoang, T., \& Lazarian, A. 2009, ApJ, 697, 1316

Holland, W. S., Bintley, D., Chapin, E. L., et al. 2013, MNRAS, 430, 2513

Holland, W. S., Greaves, J. S., Ward-Thompson, D., \& Andre, P. 1996, A\&A, 309,267

Houde, M. 2004, ApJL, 616, L111

Houde, M., Hull, C. L. H., Plambeck, R. L., Vaillancourt, J. E., \& Hildebrand, R. H. 2016, ApJ, 820, 38

Houde, M., Vaillancourt, J. E., Hildebrand, R. H., Chitsazzadeh, S., \& Kirby, L. 2009, ApJ, 706, 1504

Johnstone, D., Wilson, C. D., Moriarty-Schieven, G., et al. 2000, ApJ, 545, 327

Kandori, R., Tamura, M., Kusakabe, N., et al. 2007, PASJ, 59, 487

Kauffmann, J. 2007, PhD thesis, Univ. Bonn

Knude, J., \& Hog, E. 1998, A\&A, 338, 897

Kusune, T., Sugitani, K., Miao, J., et al. 2015, ApJ, 798, 60

Kwon, J., Choi, M., Pak, S., et al. 2010, ApJ, 708, 758

Kwon, J., Tamura, M., Hough, J. H., et al. 2015, ApJS, 220, 17

Kwon, J., Tamura, M., Kandori, R., et al. 2011, ApJ, 741, 35

Lai, S.-P., Crutcher, R. M., Girart, J. M., \& Rao, R. 2002, ApJ, 566, 925

Lazarian, A. 2007, JQSRT, 106, 225

Lazarian, A., Andersson, B.-G., \& Hoang, T. 2015, in Polarimetry of Stars and Planetary Systems, ed. L. Kolokolova, J. Hough, \& A. Levasseur-Regourd (Cambridge: Cambridge Univ. Press), 81

Loinard, L., Torres, R. M., Mioduszewski, A. J., \& Rodríguez, L. F. 2008 , ApJL, 675, L29

Lombardi, M., Lada, C. J., \& Alves, J. 2008, A\&A, 480, 785

Looney, L. W., Mundy, L. G., \& Welch, W. J. 2000, ApJ, 529, 477

Loren, R. B. 1989a, ApJ, 338, 902

Loren, R. B. 1989b, ApJ, 338, 925

Loren, R. B., \& Wootten, A. 1986, ApJ, 306, 142

Loren, R. B., Wootten, A., \& Wilking, B. A. 1990, ApJ, 365, 269

Mairs, S., Johnstone, D., Kirk, H., et al. 2015, MNRAS, 454, 2557

Mamajek, E. E. 2008, AN, 329, 10

Matthews, B. C., McPhee, C. A., Fissel, L. M., \& Curran, R. L. 2009, ApJS, 182,143
Matthews, B. C., Wilson, C. D., \& Fiege, J. D. 2001, ApJ, 562, 400

Matthews, T. G., Ade, P. A. R., Angilè, F. E., et al. 2014, ApJ, 784, 116

McKee, C. F., \& Ostriker, E. C. 2007, ARA\&A, 45, 565

Miesch, M. S., Scalo, J., \& Bally, J. 1999, ApJ, 524, 895

Motte, F., Andre, P., \& Neri, R. 1998, A\&A, 336, 150

Myers, P. C., \& Goodman, A. A. 1988, ApJ, 329, 392

Nagai, T., Inutsuka, S.-i., \& Miyama, S. M. 1998, ApJ, 506, 306

Nakamura, F., Kamada, Y., Kamazaki, T., et al. 2011, ApJ, 726, 46

Nakamura, F., Takakuwa, S., \& Kawabe, R. 2012, ApJL, 758, L25

Novak, G., Dotson, J. L., \& Li, H. 2009, ApJ, 695, 1362

Ortiz-León, G. N., Loinard, L., Kounkel, M. A., et al. 2017, ApJ, 834, 141

Ostriker, E. C., Stone, J. M., \& Gammie, C. F. 2001, ApJ, 546, 980

Palmeirim, P., André, P., Kirk, J., et al. 2013, A\&A, 550, A38

Pattle, K., Ward-Thompson, D., Berry, D., et al. 2017, ApJ, 846, 122

Pattle, K., Ward-Thompson, D., Kirk, J. M., et al. 2015, MNRAS, 450, 1094

Planck Collaboration, Ade, P. A. R., Aghanim, N., et al. 2016, A\&A, 586, A138

Poidevin, F., \& Bastien, P. 2006, ApJ, 650, 945

Rebull, L. M., Wolff, S. C., \& Strom, S. E. 2004, AJ, 127, 1029

Santos, F., Dowell, C. D., Houde, M., et al. 2018, in American Astronomical Society Meeting Abstracts 231, 130.04

Santos, F. P., Franco, G. A. P., Roman-Lopes, A., Reis, W., \& Román-Zúñiga, C. G. 2014, ApJ, 783, 1

Sato, S., Tamura, M., Nagata, T., et al. 1988, MNRAS, 230, 321

Shu, F. H., Adams, F. C., \& Lizano, S. 1987, ARA\&A, 25, 23

Snow, T. P., Destree, J. D., \& Welty, D. E. 2008, ApJ, 679, 512

Soler, J. D., Alves, F., Boulanger, F., et al. 2016, A\&A, 596, A93

Sugitani, K., Nakamura, F., Watanabe, M., et al. 2011, ApJ, 734, 63

Tamura, M. 1999, in Star Formation 1999, 212

Tamura, M., Hayashi, S., Itoh, Y., Hough, J. H., \& Chrysostomou, A. 1996, in ASP Conf. Ser. 97, Polarimetry of the Interstellar Medium, ed. W. G. Roberge \& D. C. B. Whittet (San Francisco, CA: ASP), 372

Tamura, M., Hough, J. H., Greaves, J. S., et al. 1999, ApJ, 525, 832

Tamura, M., Kandori, R., Hashimoto, J., et al. 2007, PASJ, 59, 467

Tamura, M., Nagata, T., Sato, S., \& Tanaka, M. 1987, MNRAS, 224, 413

Tamura, M., Yamashita, T., Sato, S., Nagata, T., \& Gatley, I. 1988, MNRAS, 231,445

Umemoto, T., Mikami, H., Yamamoto, S., \& Hirano, N. 1999, ApJL, 525, L105

Vaillancourt, J. E. 2006, PASP, 118, 1340

van Kempen, T. A., van Dishoeck, E. F., Salter, D. M., et al. 2009, A\&A, 498, 167

Vrba, F. J. 1977, AJ, 82, 198

Vrba, F. J., Strom, S. E., \& Strom, K. M. 1976, AJ, 81, 958

Ward-Thompson, D., Di Francesco, J., Hatchell, J., et al. 2007, PASP, 119, 855

Ward-Thompson, D., Kirk, J. M., Greaves, J. S., \& André, P. 2011, MNRAS, 415,2812

Ward-Thompson, D., Pattle, K., Bastien, P., et al. 2017a, ApJ, 842, 66

Ward-Thompson, D., Pattle, K., Kirk, J. M., Andre, P., \& Di Francesco, J. 2017b, PKAS, 32, 117

Ward-Thompson, D., Robson, E. I., Whittet, D. C. B., et al. 1989, MNRAS, 241,119

White, G. J., Drabek-Maunder, E., Rosolowsky, E., et al. 2015, MNRAS, 447, 1996

Wilking, B. A., Gagné, M., \& Allen, L. E. 2008, in Handbook of Star Forming Regions, Vol. II, ed. B. Reipurth (San Francisco, CA: ASP), 351

Wilking, B. A., Lebofsky, M. J., Kemp, J. C., \& Rieke, G. H. 1979, AJ, 84, 199

Wilson, C. D., Avery, L. W., Fich, M., et al. 1999, ApJL, 513, L139

Yu, T., \& Chernin, L. M. 1997, ApJL, 479, L63

Zeng, Q., Batrla, W., \& Wilson, T. L. 1984, A\&A, 141, 127 